\title{
TIPS-Diazoacetone Aldol Addition: Mechanistic Aspects and Contribution to the Synthesis
}

Sigrid Gavelle, Imen Abid, Bohdan Biletskyi, Sylvain Henrion, Annie Hémon-Ribaud, Jérôme Lhoste, Arnaud Martel, Gilles Dujardin and Catherine Gaulon-Nourry*

Institut des Molécules et Matériaux du Mans, IMMM UMR 6283 CNRS - Le Mans Université, Avenue Olivier Messiaen, 72085 Le Mans CEDEX 9, France.

E.mail: catherine.gaulon@univ-lemans.fr

\section{SUPPORTING INFORMATION}

\section{Table of Contents}
${ }^{1} \mathrm{H},{ }^{13} \mathrm{C}$ and ${ }^{19} \mathrm{~F}$ NMR spectra for all new compounds
Page
Crystallographic data for compounds $3 \mathbf{a}, \mathbf{3 b}, \mathbf{3 e}, \mathbf{4 a}$ and $\mathbf{1 0}$
S2-S49
Computational data
S50-S53
S54-S73 

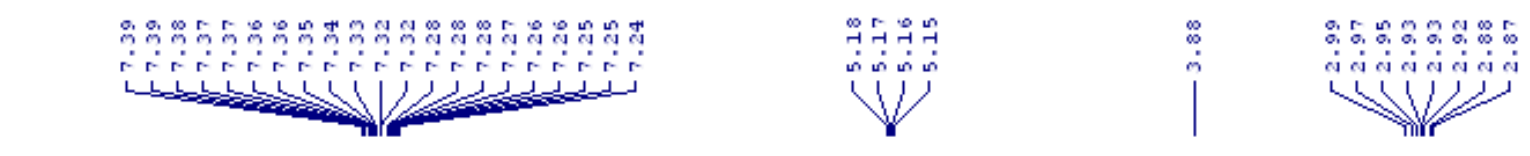

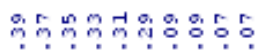

${ }^{1} \mathrm{H}\left(400 \mathrm{MHz}, \mathrm{CDCl}_{3}\right)$

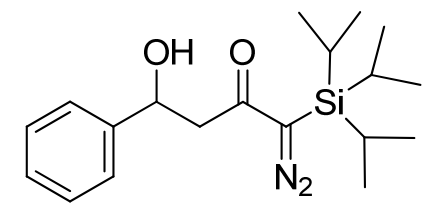

$3 a$
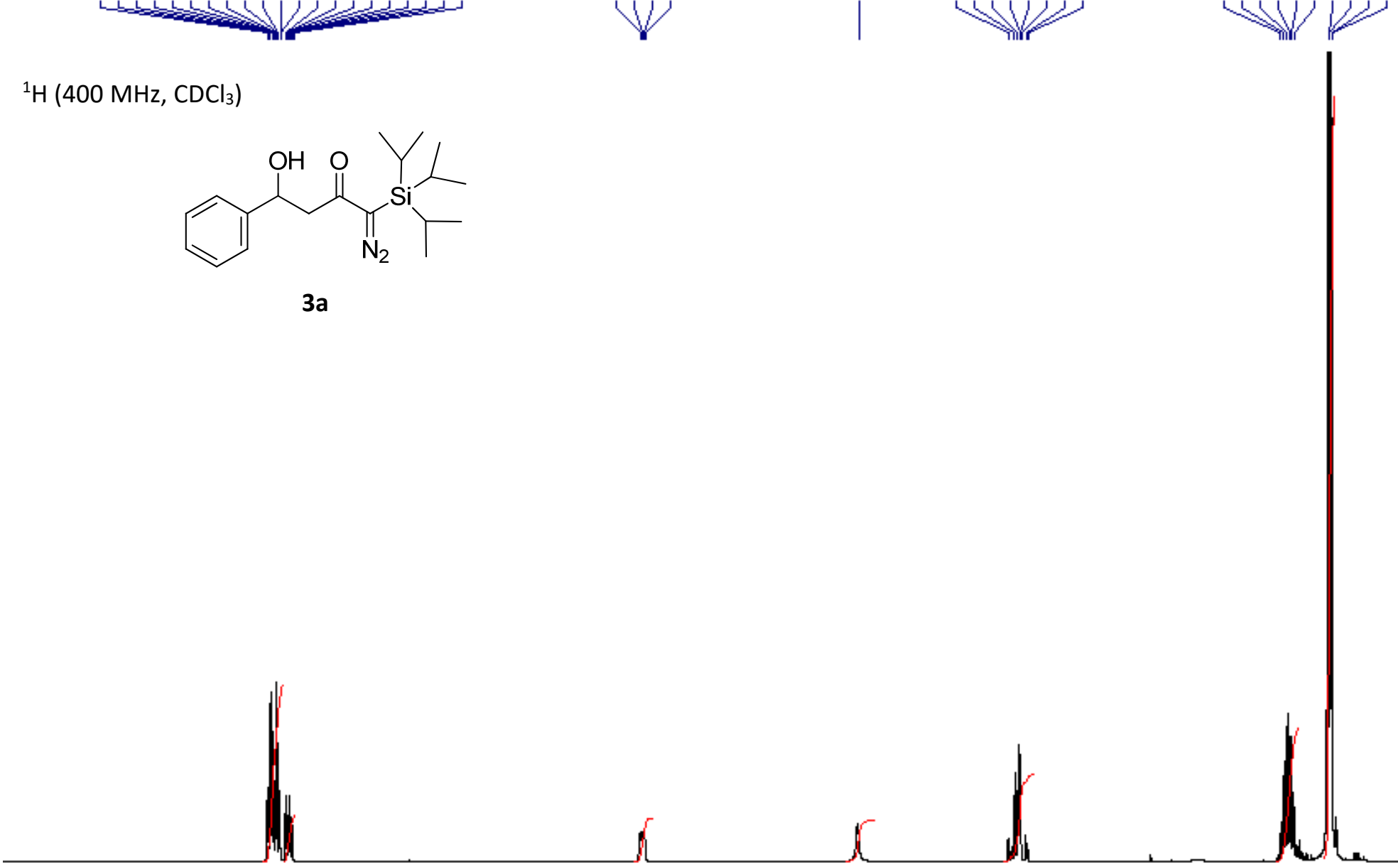

$\begin{array}{lll}8.5 & 8.0 & 7.5\end{array}$ $\left|\begin{array}{l}n \\
0 \\
0 \\
\dot{0}\end{array}\right|$\begin{tabular}{l}
0 \\
$\vdots$ \\
\hdashline
\end{tabular} \mid $|\stackrel{b}{-}|$

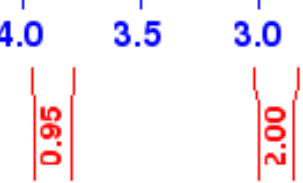

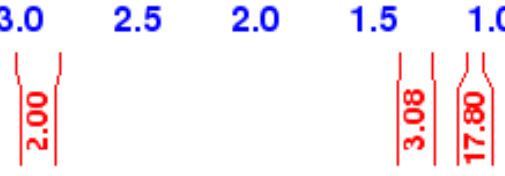




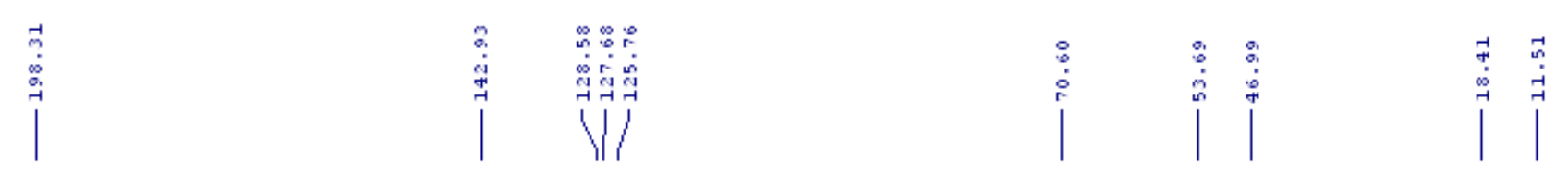

${ }^{13} \mathrm{C}\left\{{ }^{1} \mathrm{H}\right\}\left(100.6 \mathrm{MHz}, \mathrm{CDCl}_{3}\right)$

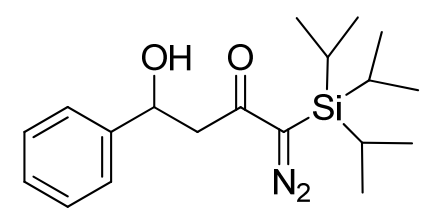

$3 a$

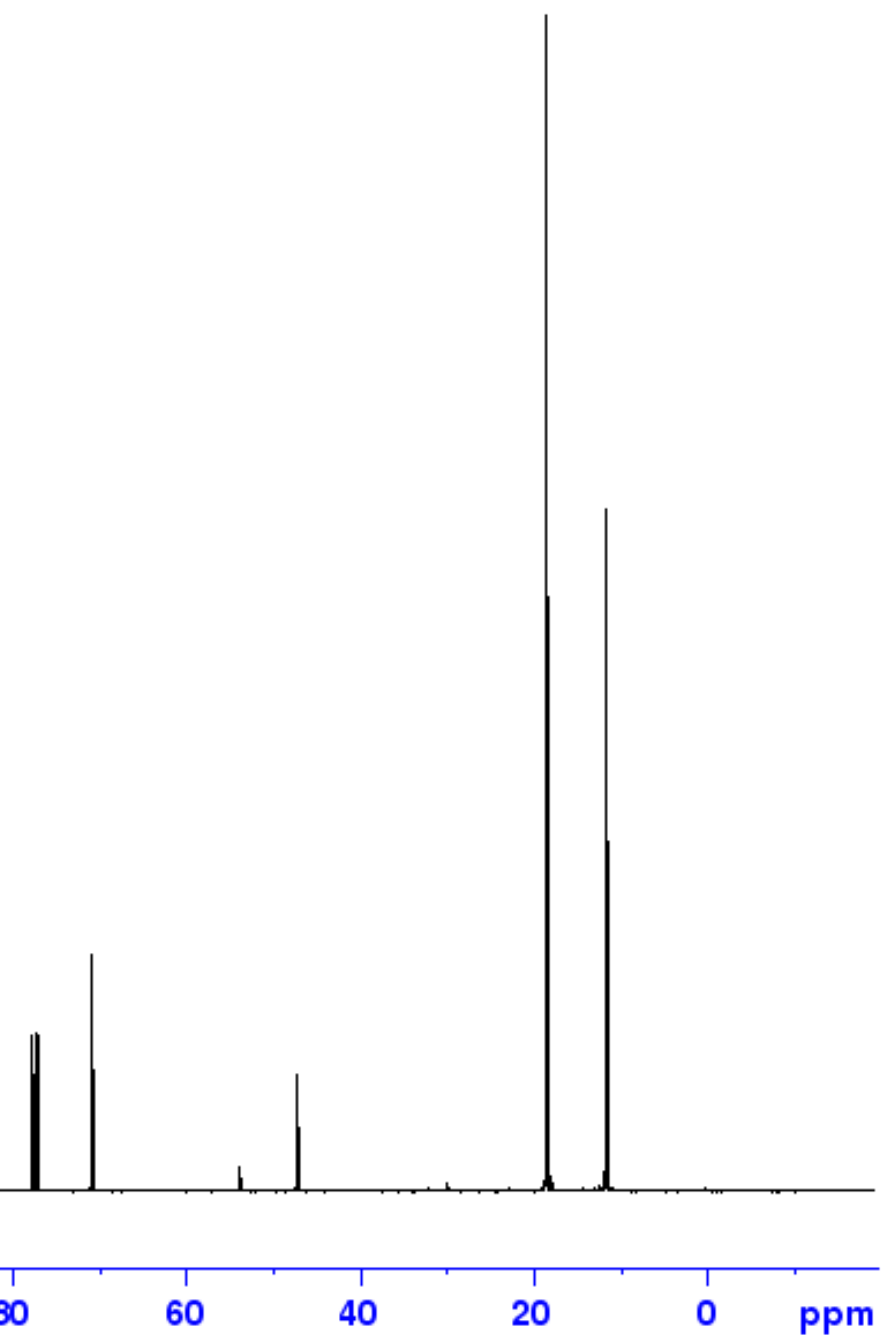



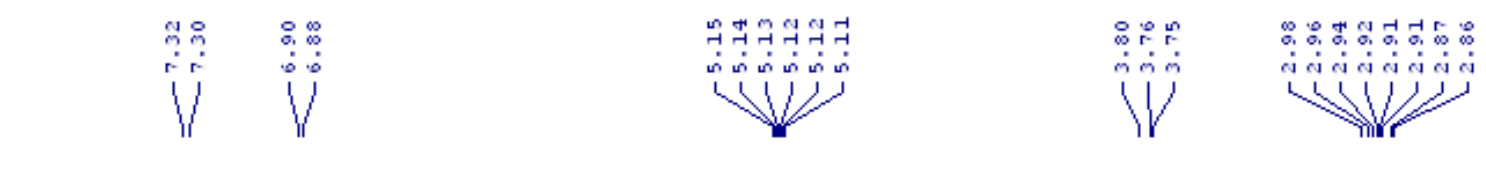

${ }^{1} \mathrm{H}\left(400 \mathrm{MHz}, \mathrm{CDCl}_{3}\right)$

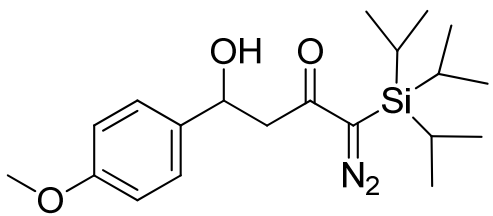

$3 b$

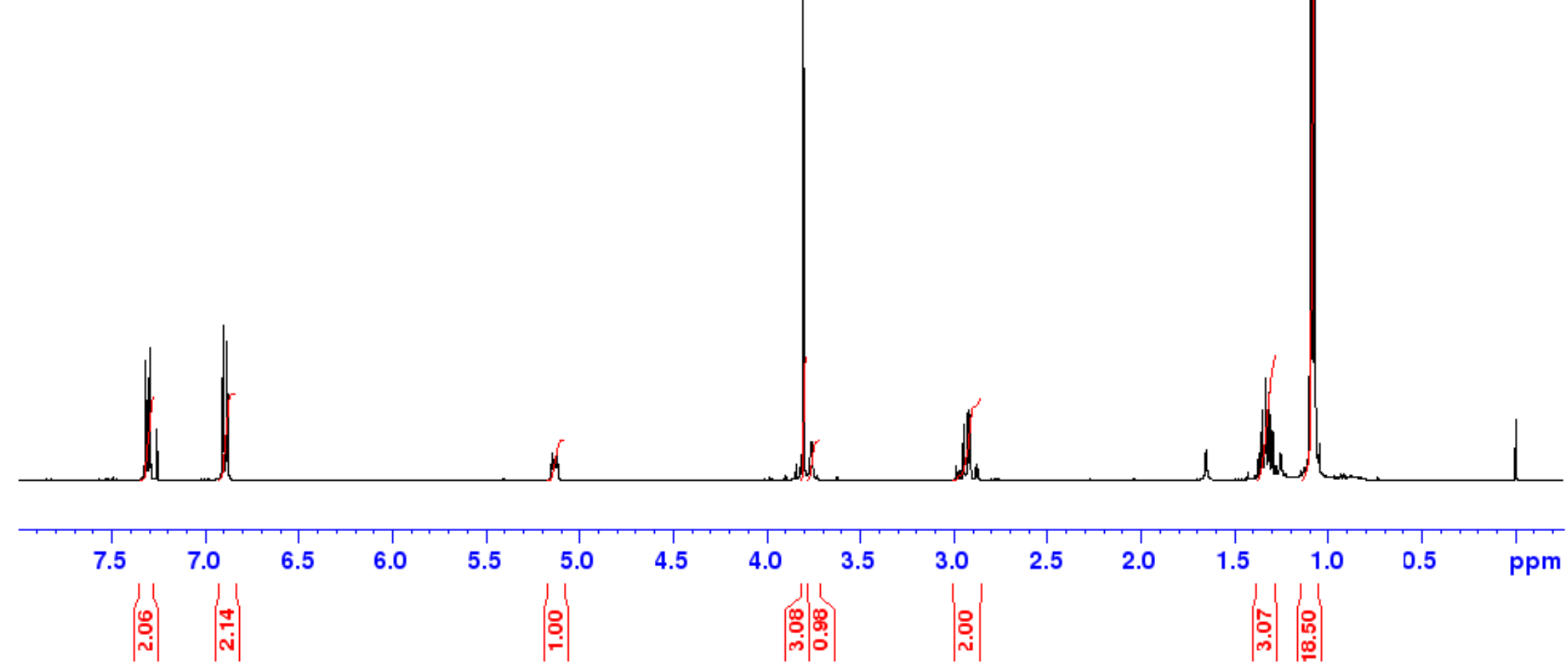




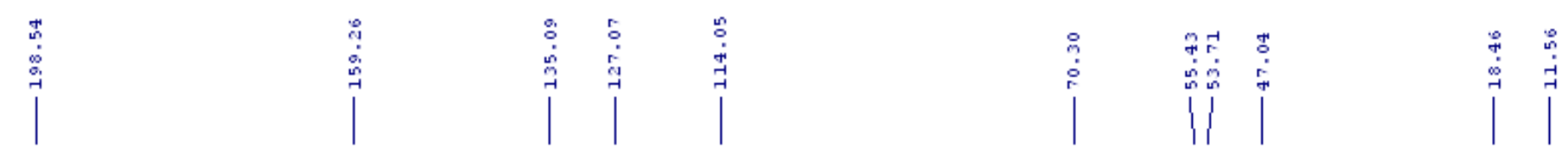

${ }^{13} \mathrm{C}\left\{{ }^{1} \mathrm{H}\right\}\left(100.6 \mathrm{MHz}, \mathrm{CDCl}_{3}\right)$

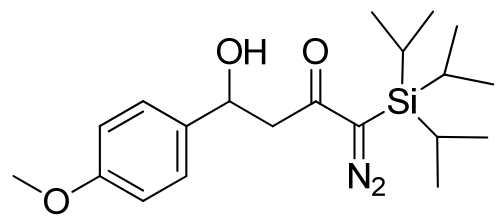

3b

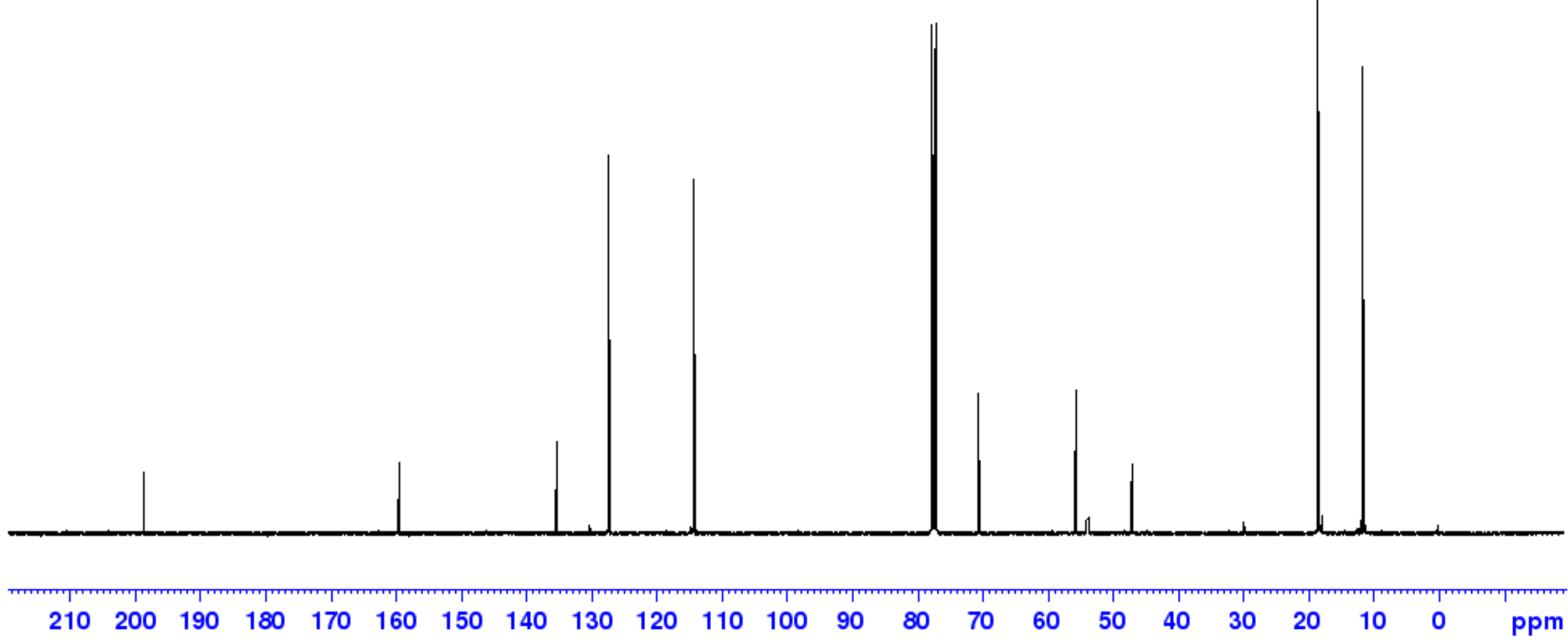




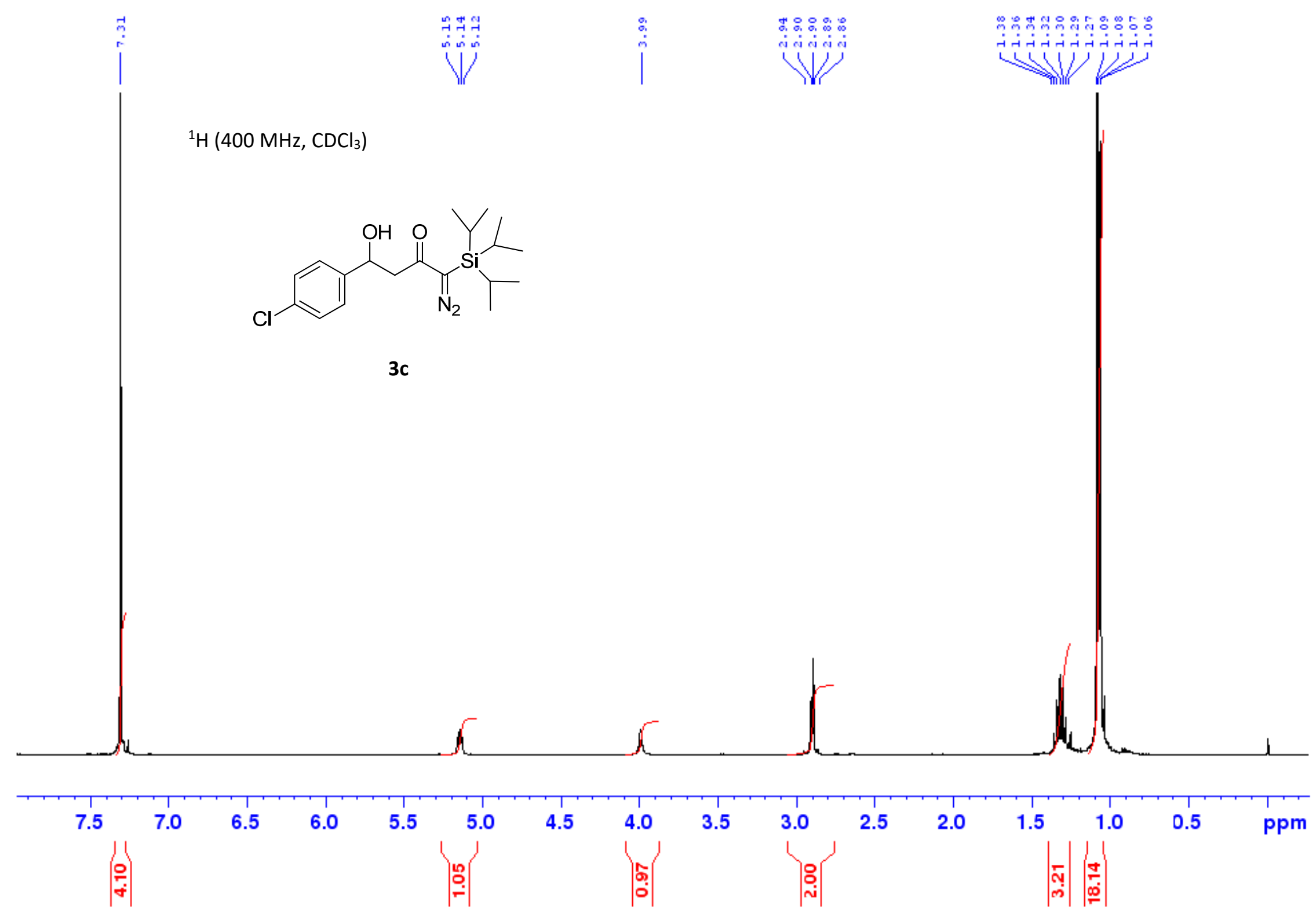



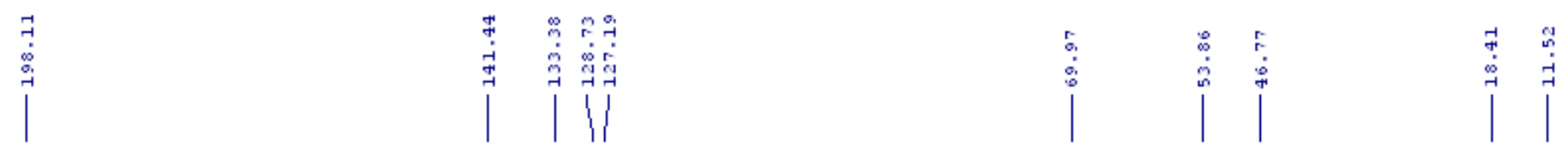

${ }^{13} \mathrm{C}\left\{{ }^{1} \mathrm{H}\right\}\left(100.6 \mathrm{MHz}, \mathrm{CDCl}_{3}\right)$
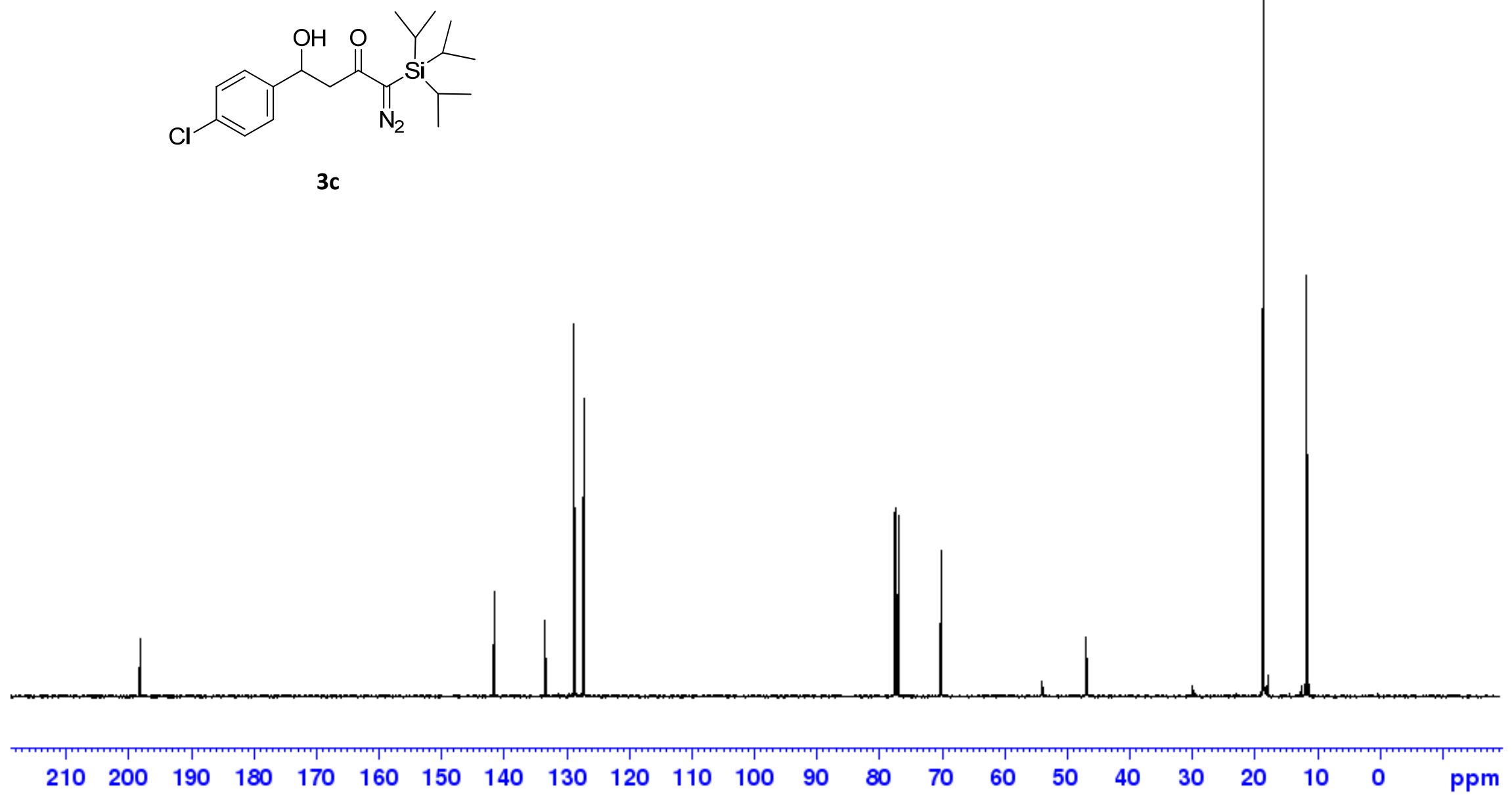


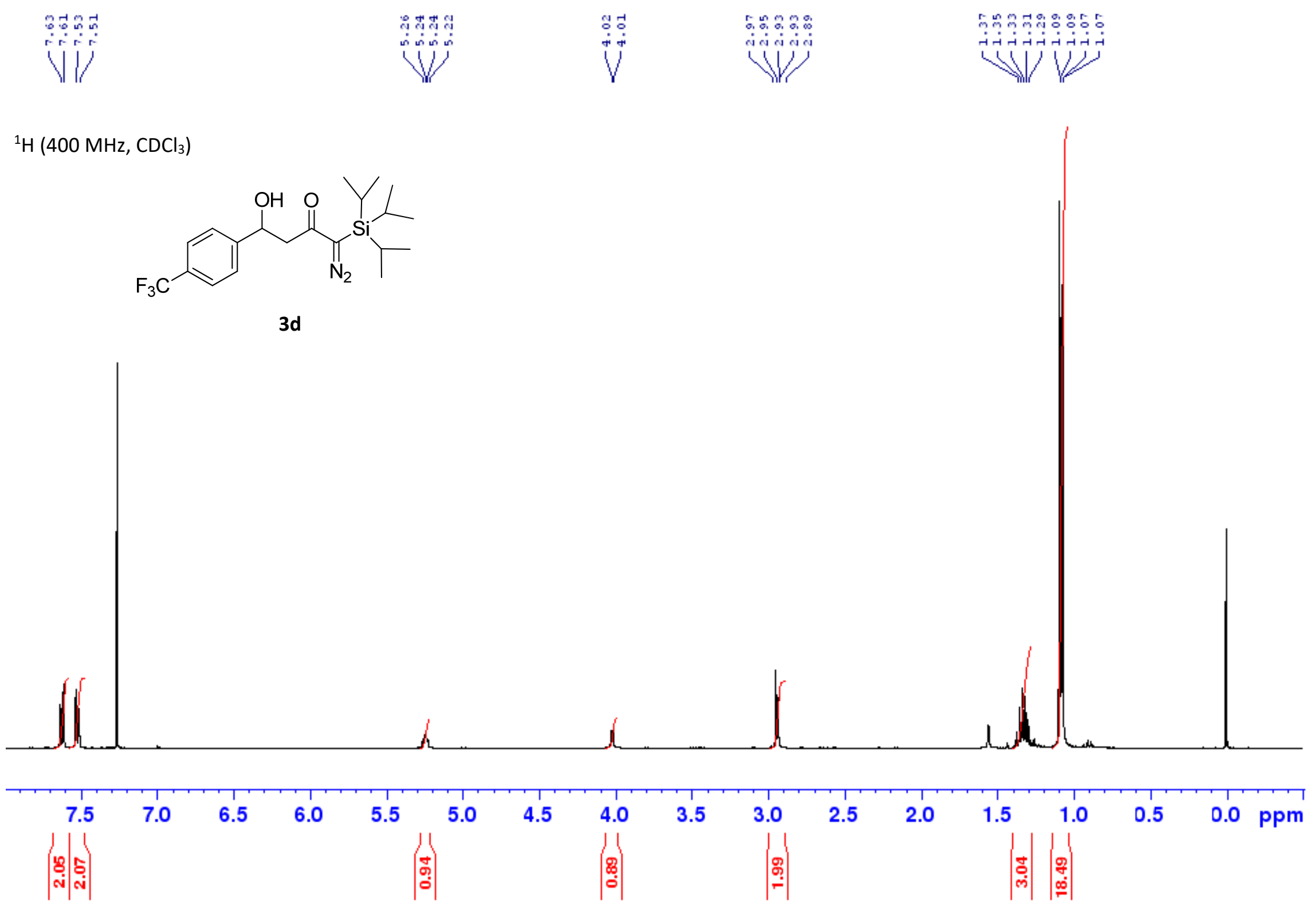




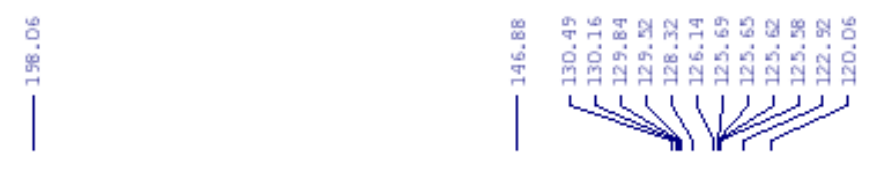

${ }^{13} \mathrm{C}\left\{{ }^{1} \mathrm{H}\right\}\left(100.6 \mathrm{MHz}, \mathrm{CDCl}_{3}\right)$
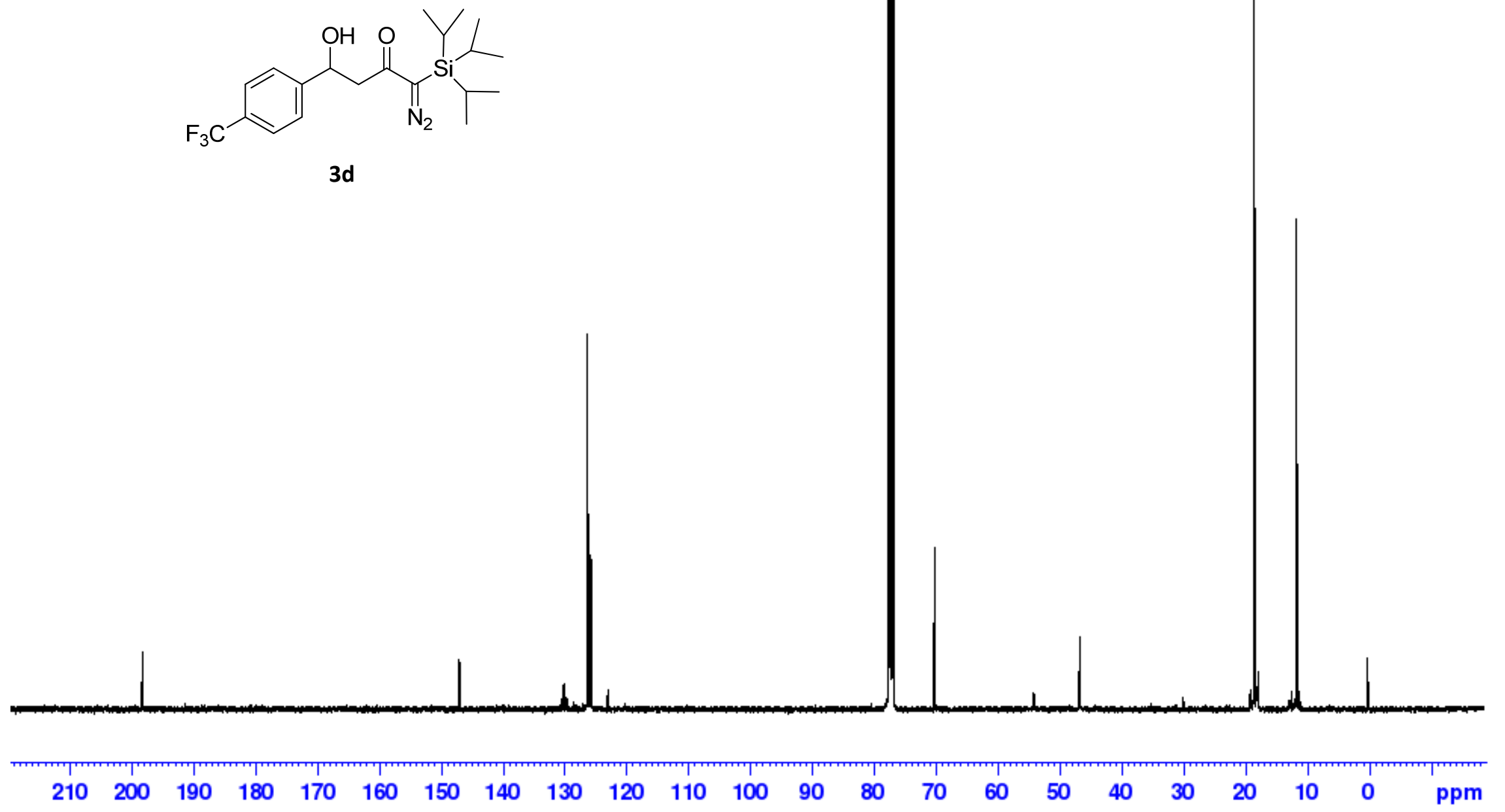
${ }^{19} \mathrm{~F}\left(188.3 \mathrm{MHz}, \mathrm{CDCl}_{3}\right)$

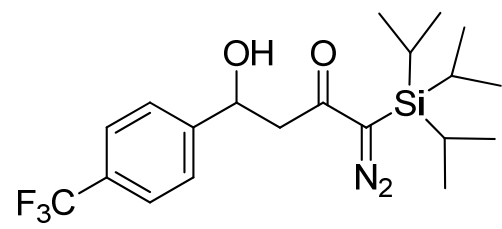

3d

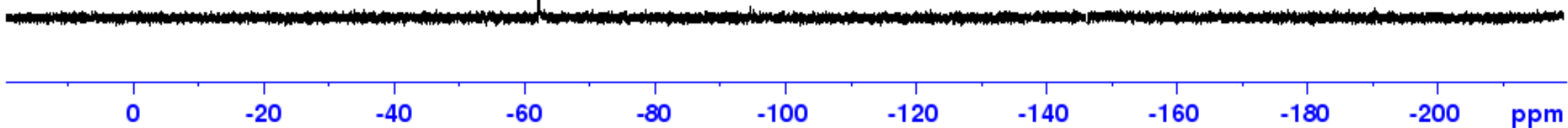




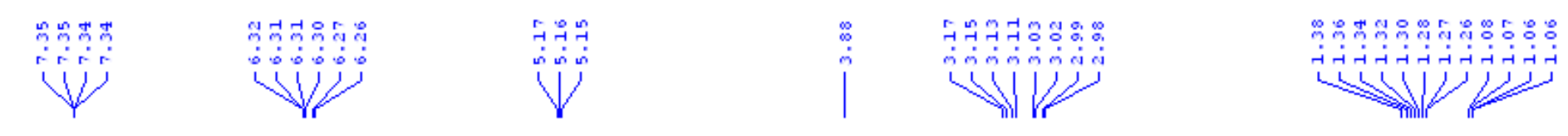

${ }^{1} \mathrm{H}\left(400 \mathrm{MHz}, \mathrm{CDCl}_{3}\right)$

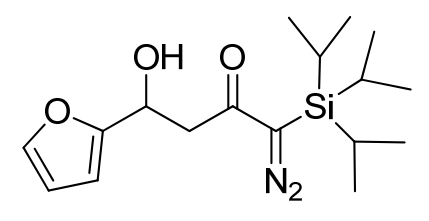

$3 e$
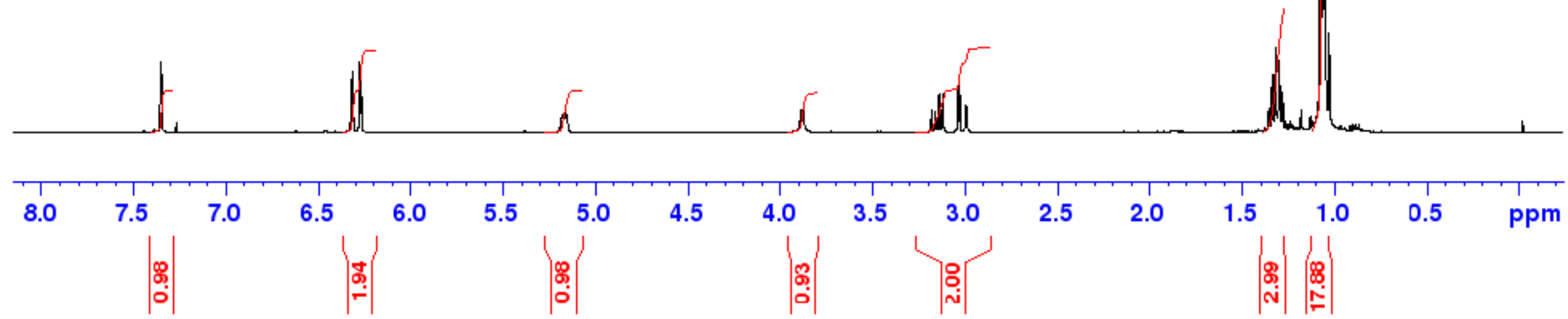


$$
1+11+11
$$

${ }^{13} \mathrm{C}\left\{{ }^{1} \mathrm{H}\right\}\left(100.6 \mathrm{MHz}, \mathrm{CDCl}_{3}\right)$

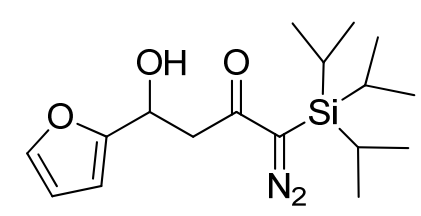

$3 e$
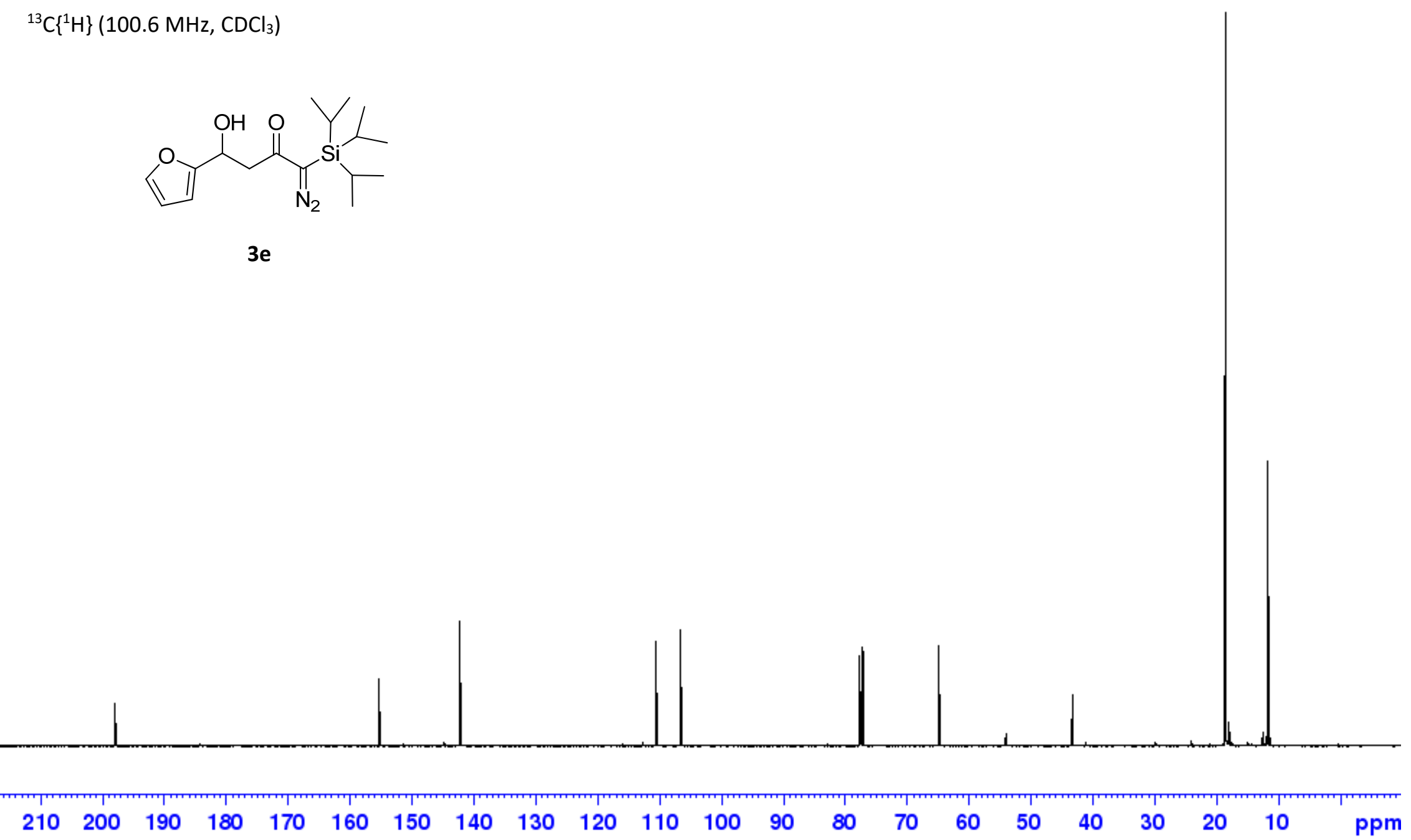


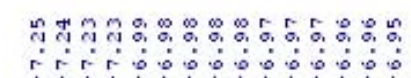

rirrioiojojijo

꿍ํํำ꾸ำ

ivi

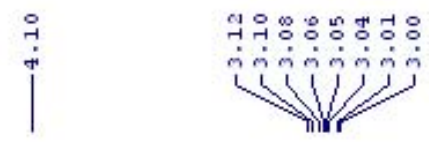

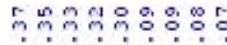

$\sqrt{1} \sqrt{1}$

${ }^{1} \mathrm{H}\left(400 \mathrm{MHz}, \mathrm{CDCl}_{3}\right)$

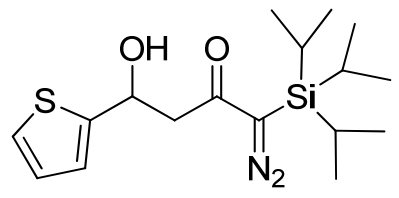

$3 f$

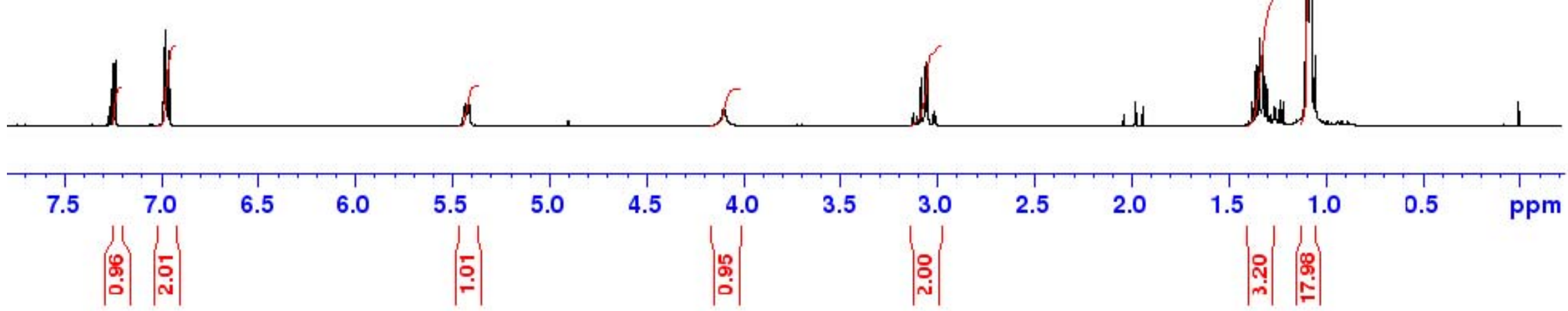




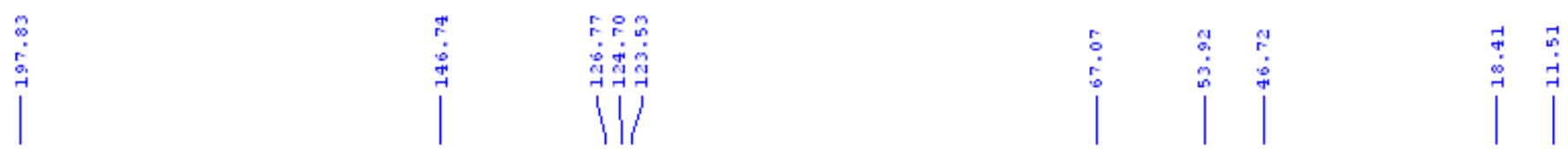

${ }^{13} \mathrm{C}\left\{{ }^{1} \mathrm{H}\right\}\left(100.6 \mathrm{MHz}, \mathrm{CDCl}_{3}\right)$

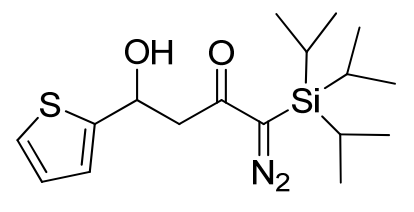

$3 f$
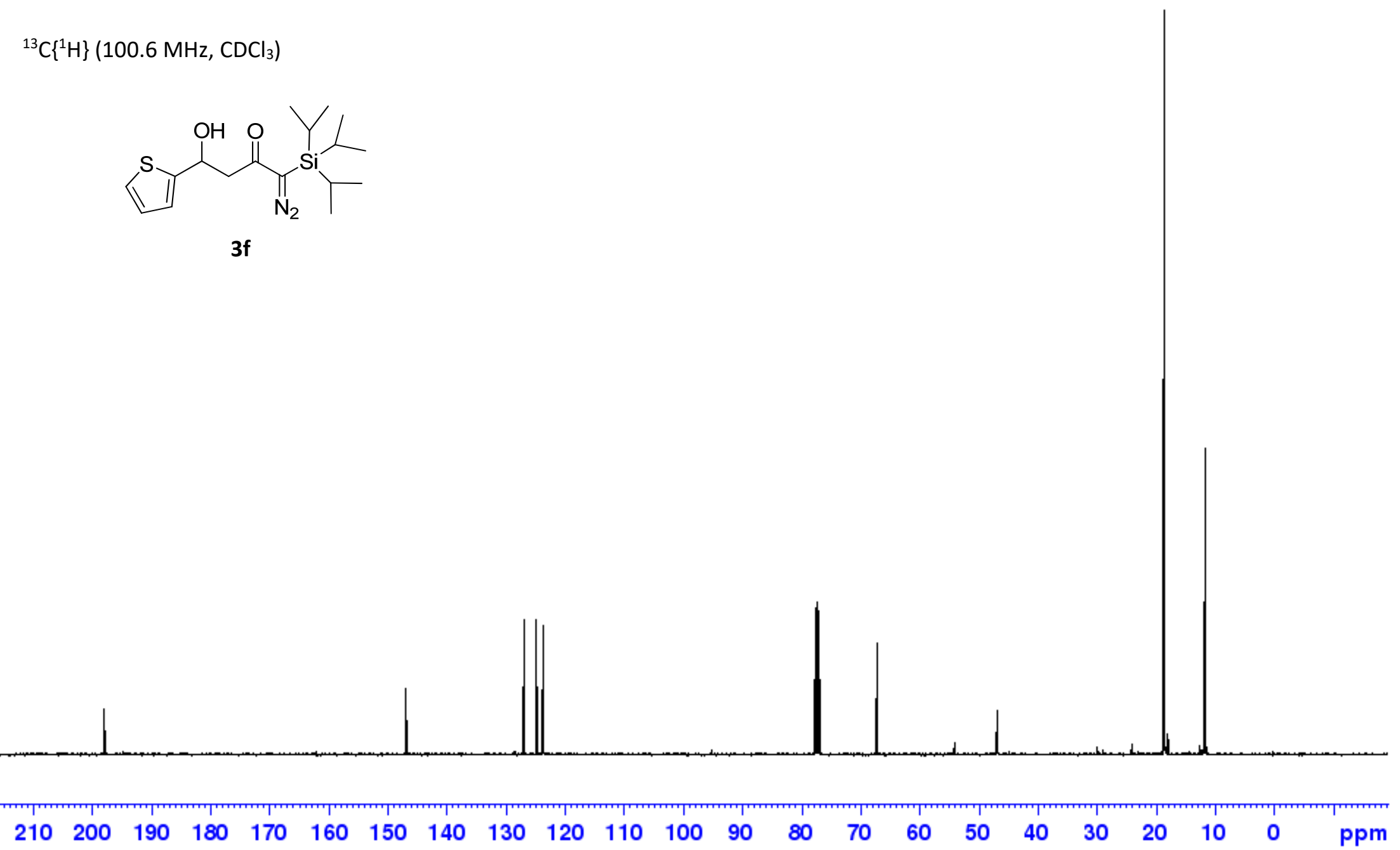
${ }^{1} \mathrm{H}\left(400 \mathrm{MHz}, \mathrm{CDCl}_{3}\right)$

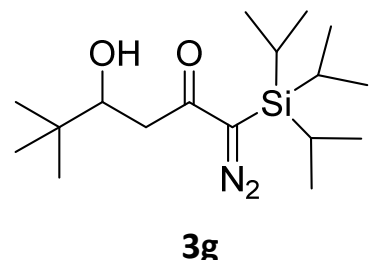

$3 g$

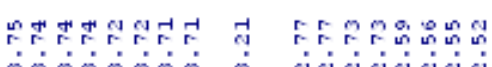

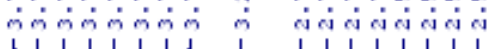

N W

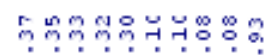

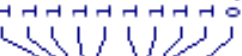

Mr
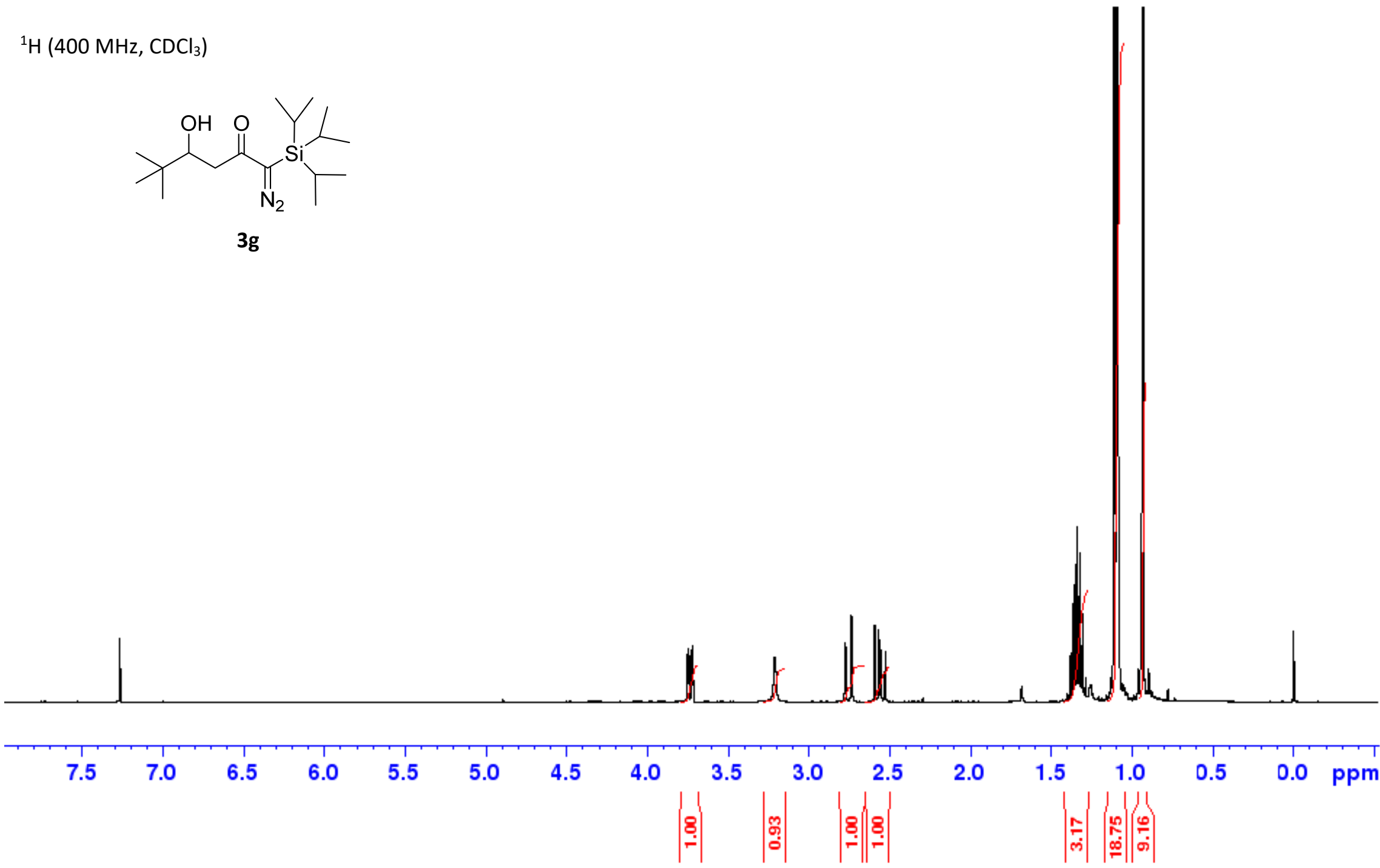
${ }^{13} \mathrm{C}\left\{{ }^{1} \mathrm{H}\right\}\left(100.6 \mathrm{MHz}, \mathrm{CDCl}_{3}\right)$

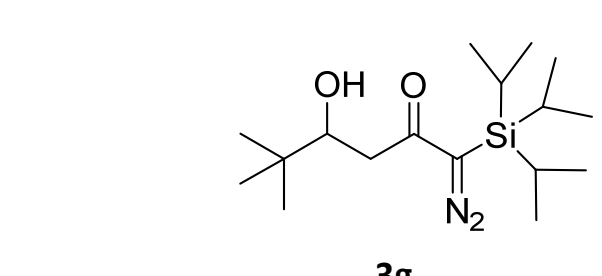

3g
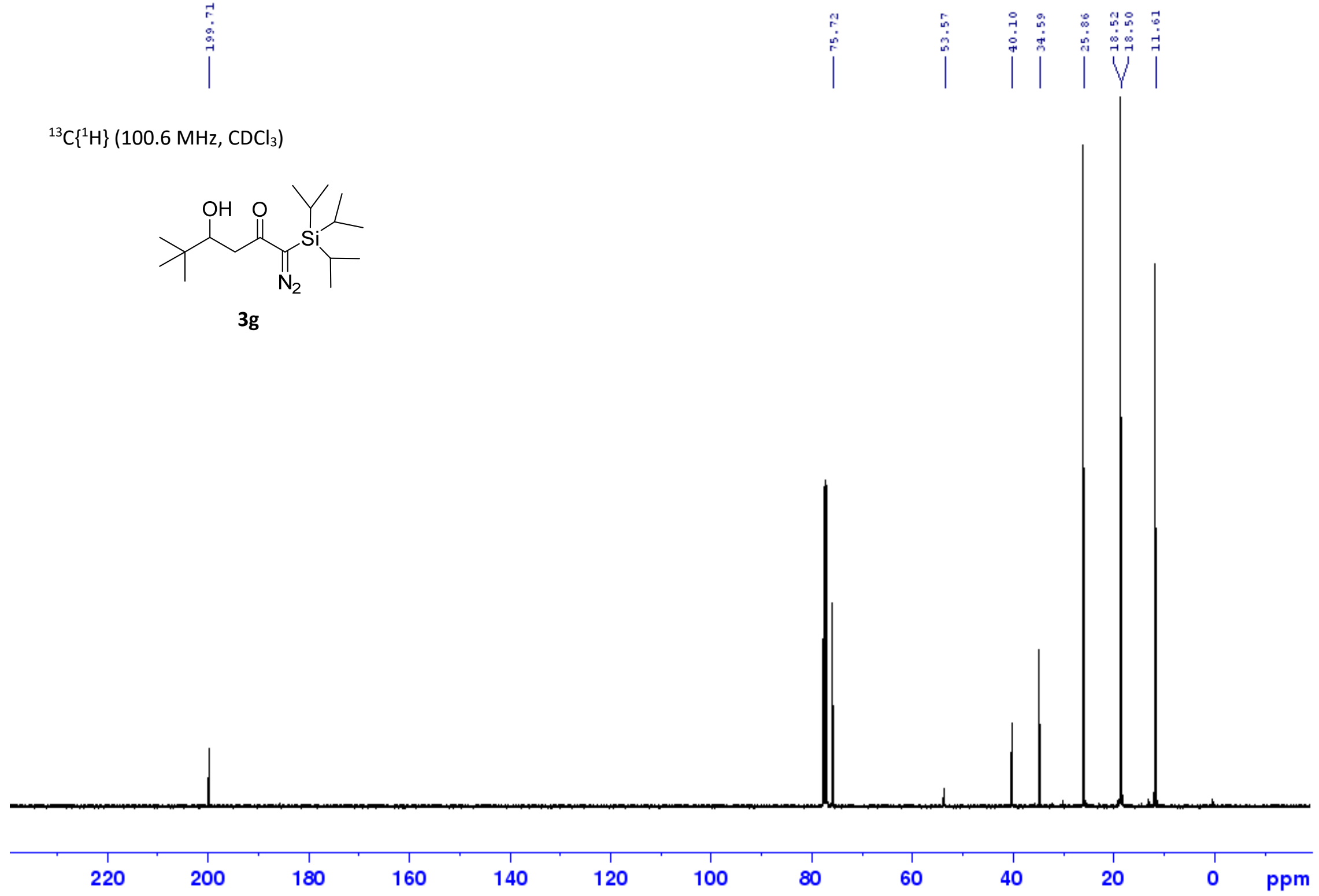


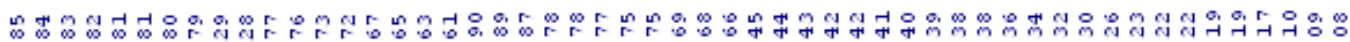

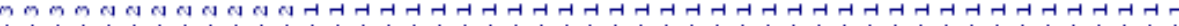

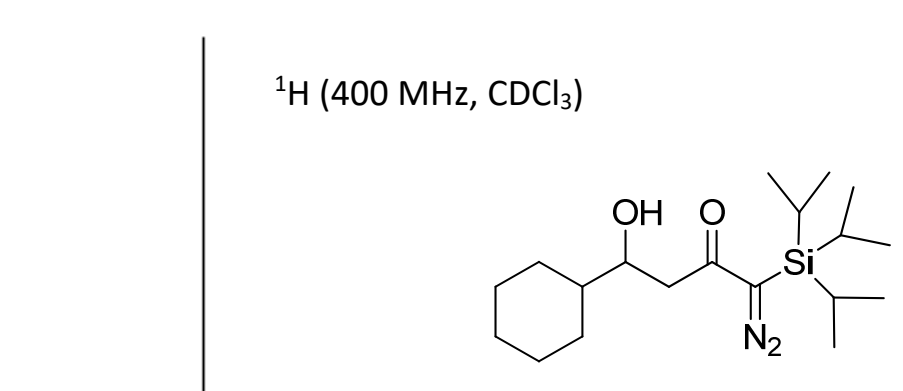

$3 h$

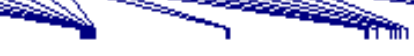

9ा 


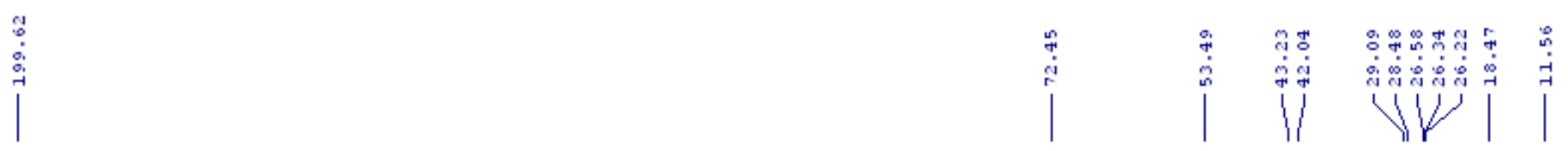

${ }^{13} \mathrm{C}\left\{{ }^{1} \mathrm{H}\right\}\left(100.6 \mathrm{MHz}, \mathrm{CDCl}_{3}\right)$

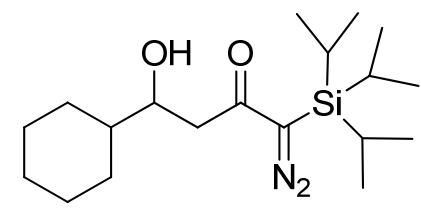

$3 h$
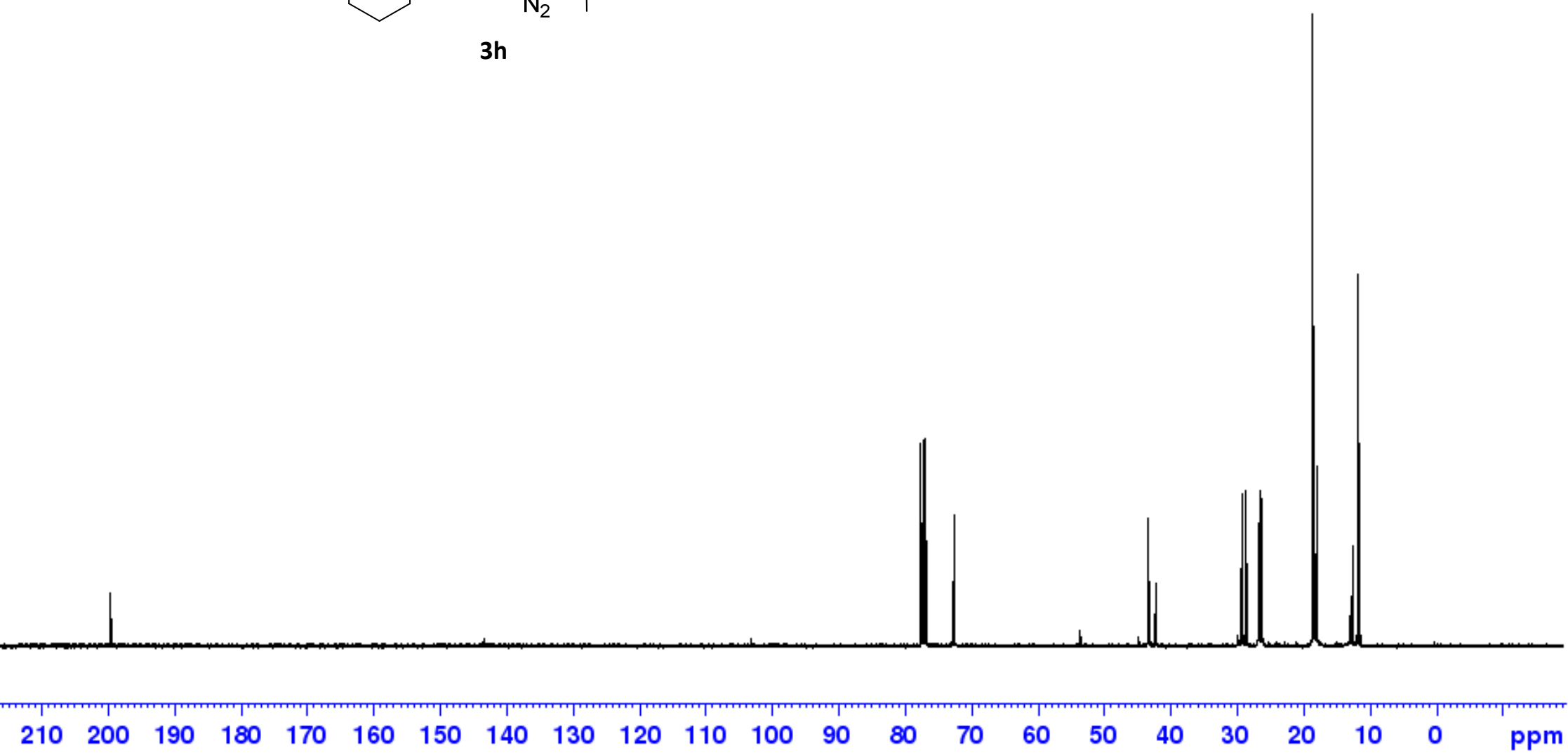
${ }^{1} \mathrm{H}\left(400 \mathrm{MHz}, \mathrm{CDCl}_{3}\right)$

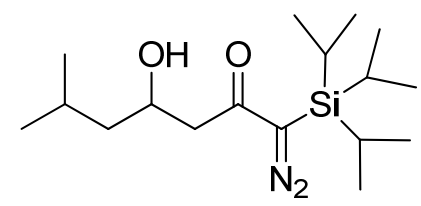

$3 \mathbf{i}$
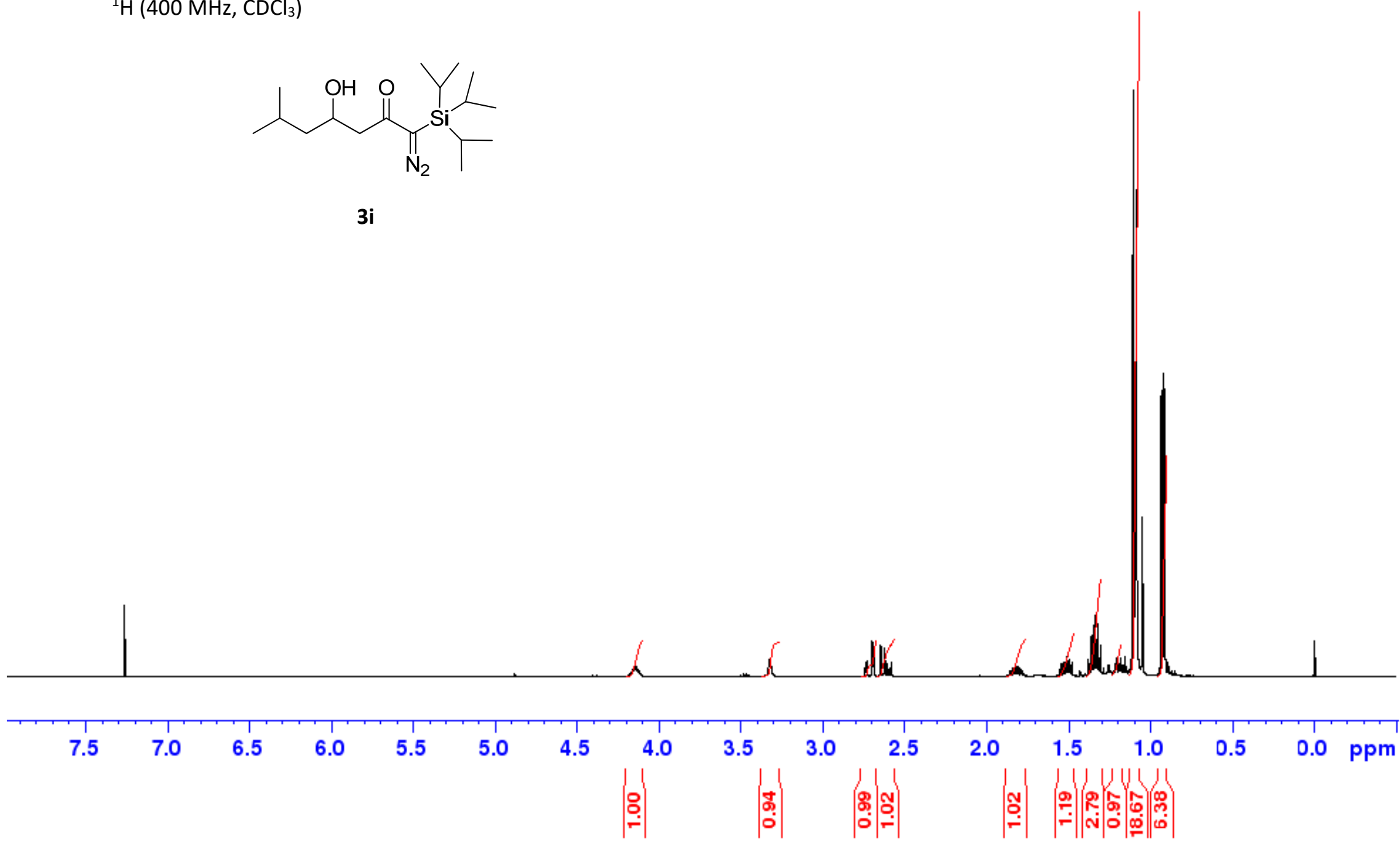
${ }^{13} \mathrm{C}\left\{{ }^{1} \mathrm{H}\right\}\left(100.6 \mathrm{MHz}, \mathrm{CDCl}_{3}\right)$

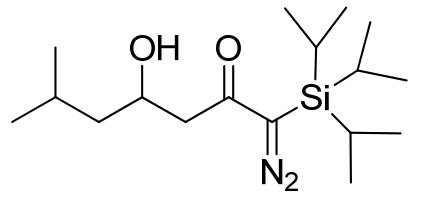

3i

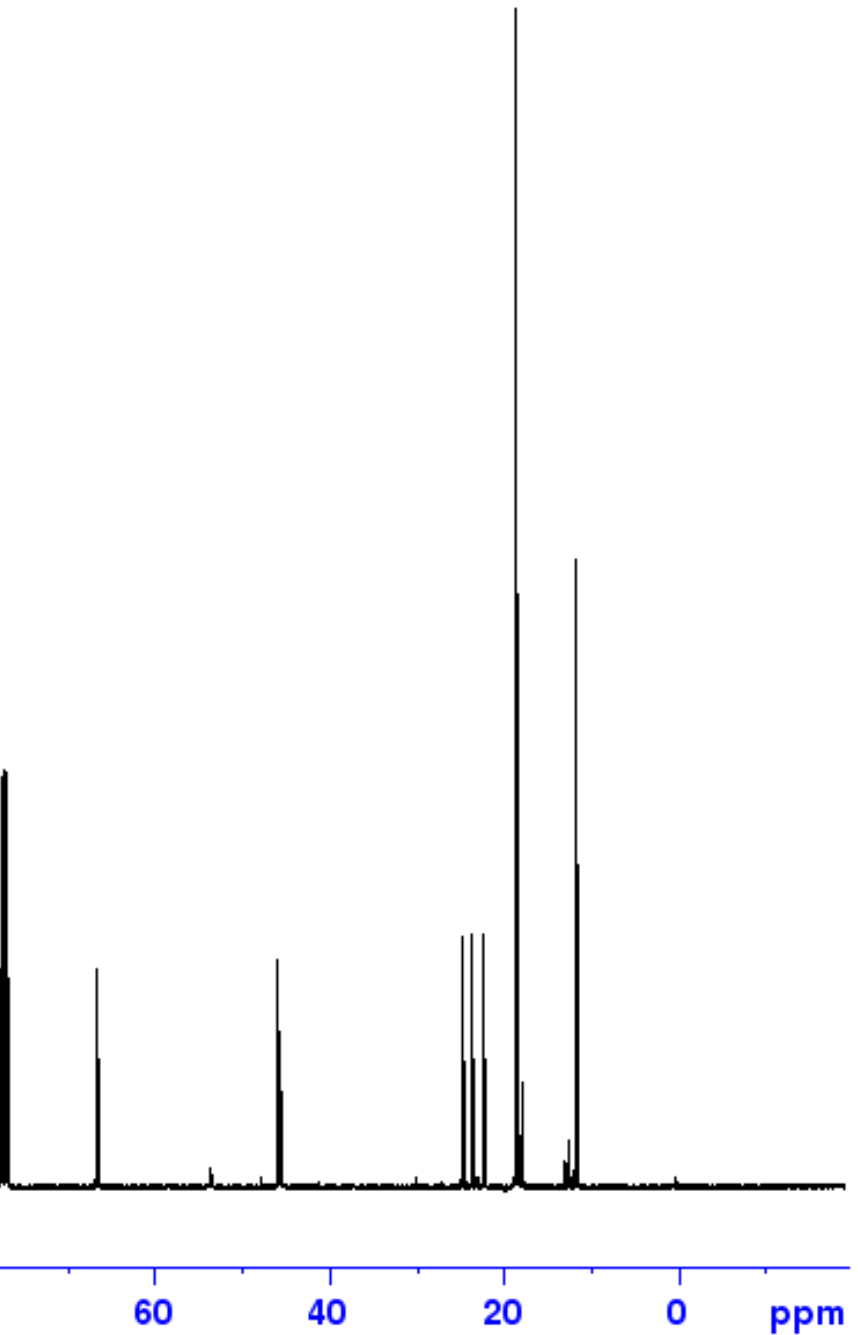


${ }^{1} \mathrm{H}\left(400 \mathrm{MHz}, \mathrm{CDCl}_{3}\right)$

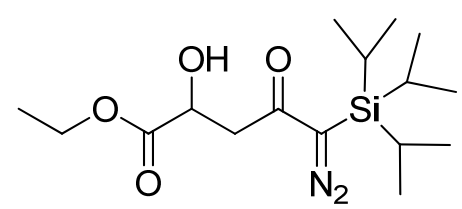

3j

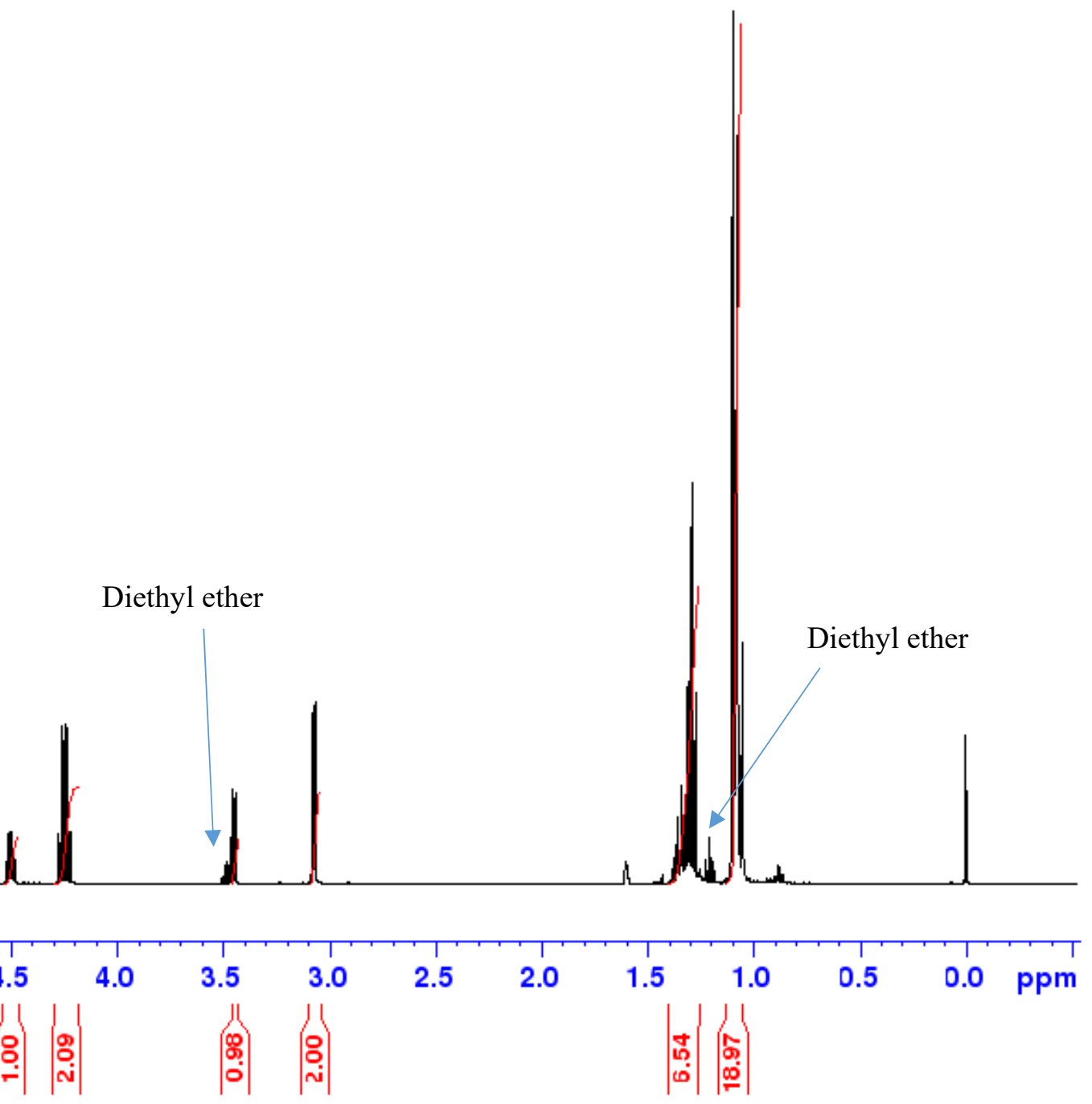



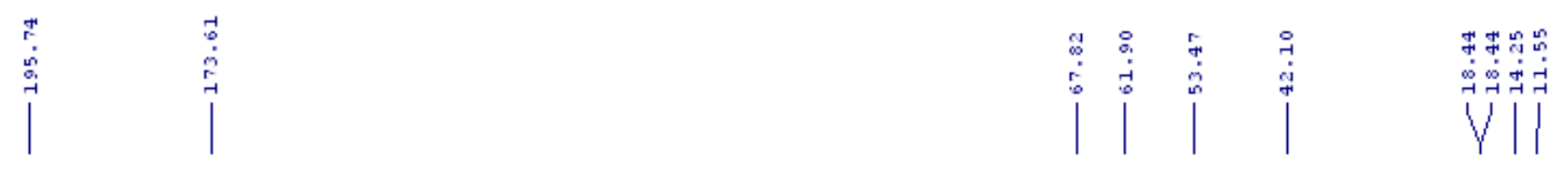

${ }^{13} \mathrm{C}\left\{{ }^{1} \mathrm{H}\right\}\left(100.6 \mathrm{MHz}, \mathrm{CDCl}_{3}\right)$

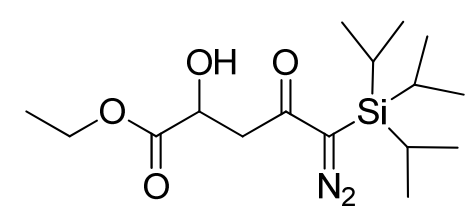

3j

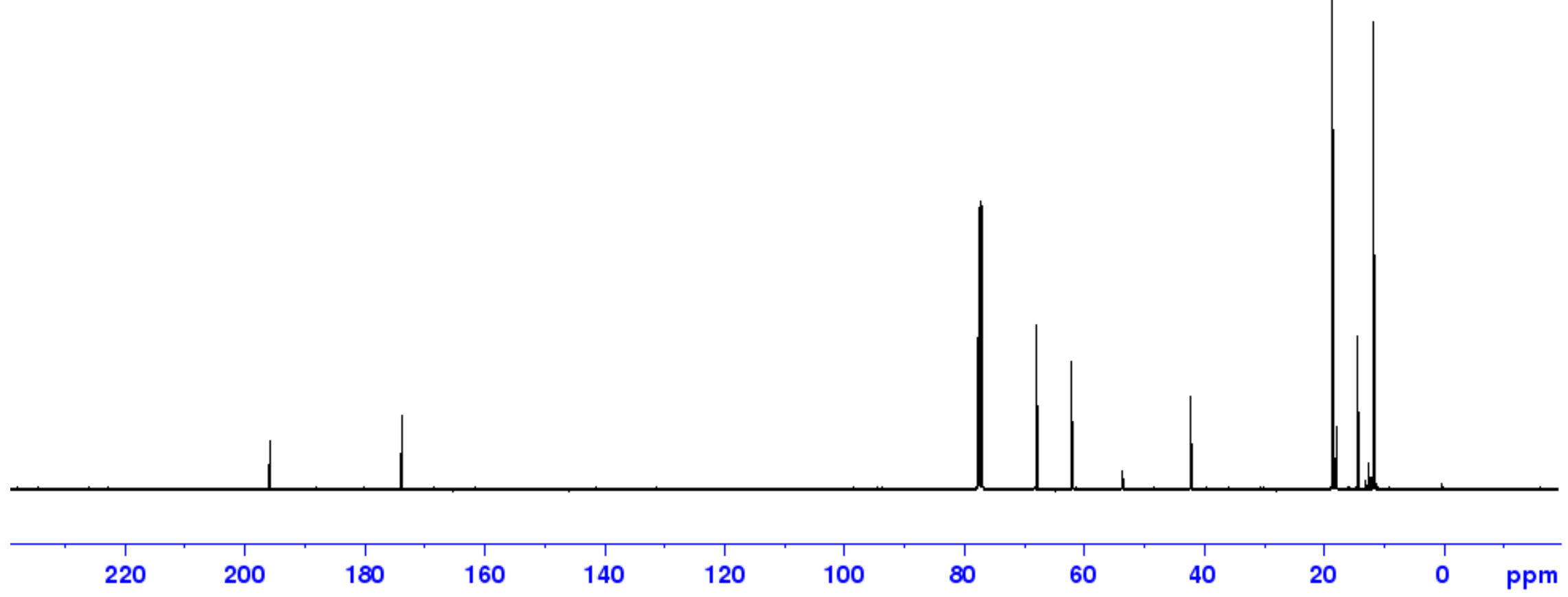


${ }^{1} \mathrm{H}\left(400 \mathrm{MHz}, \mathrm{CDCl}_{3}\right)$

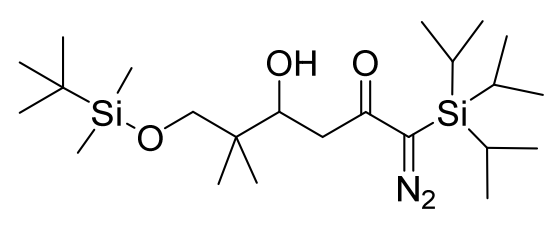

$3 k$
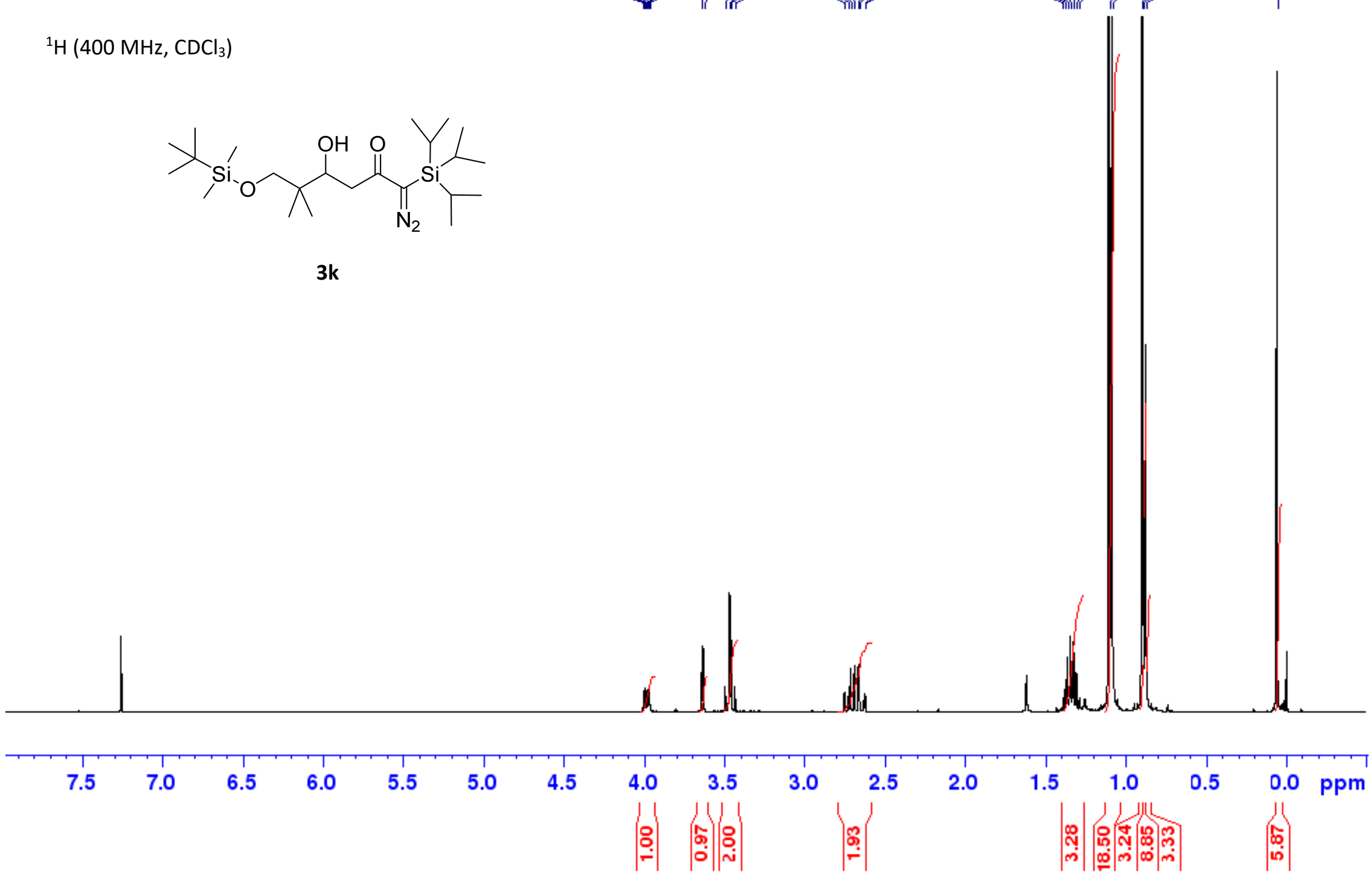
${ }^{13} \mathrm{C}\left\{{ }^{1} \mathrm{H}\right\}\left(100.6 \mathrm{MHz}, \mathrm{CDCl}_{3}\right)$

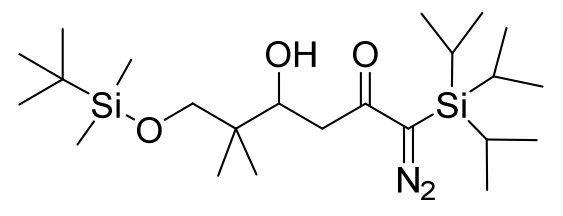

3k

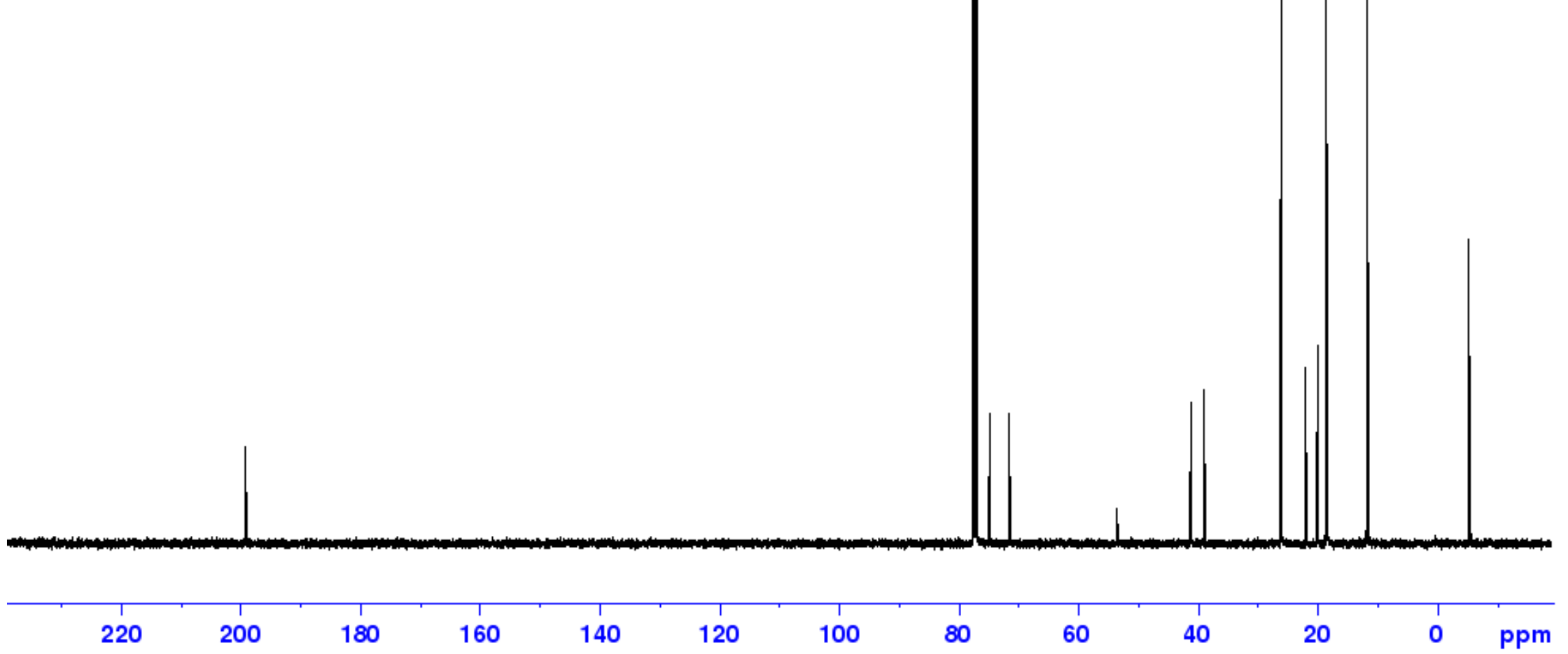




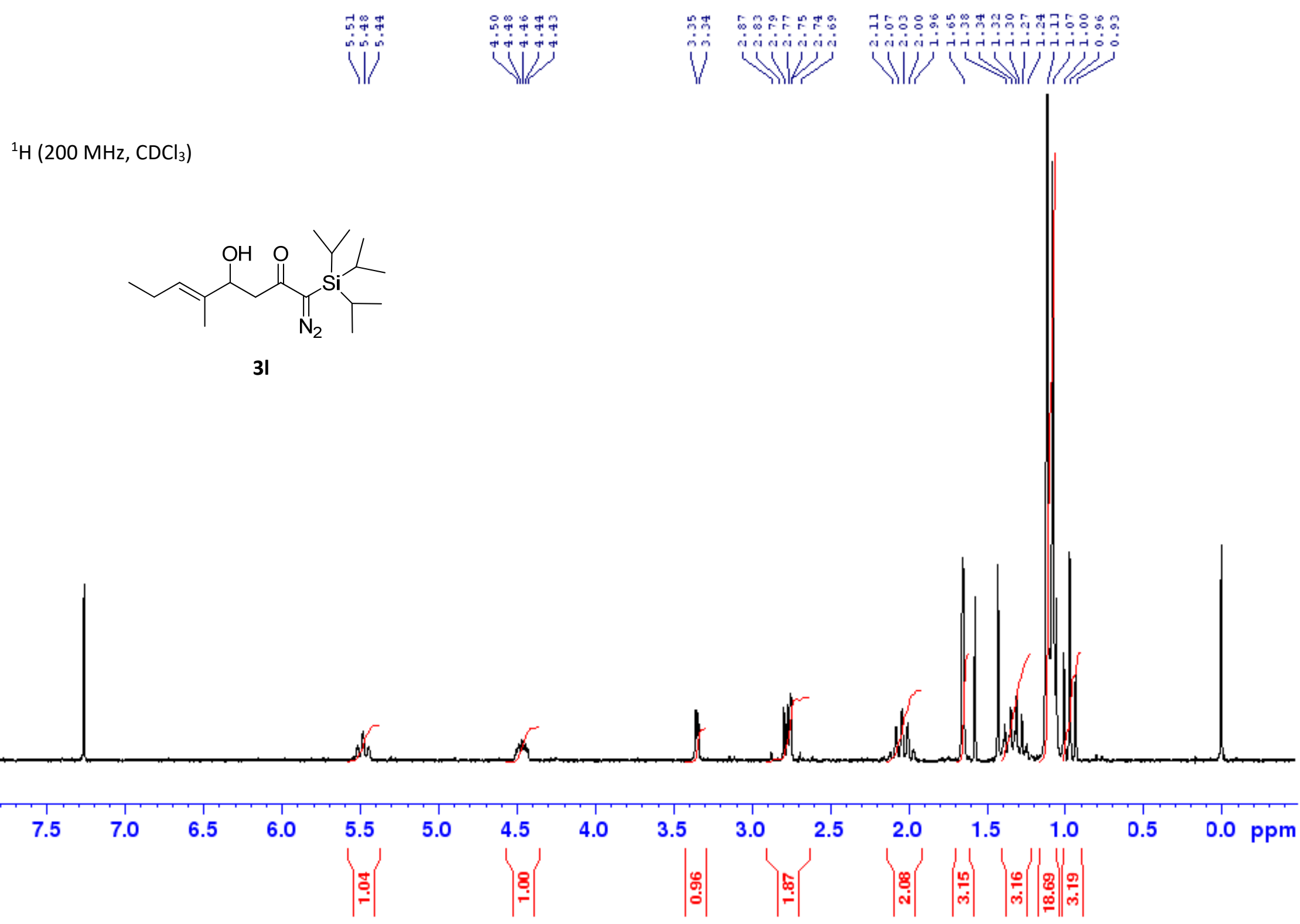




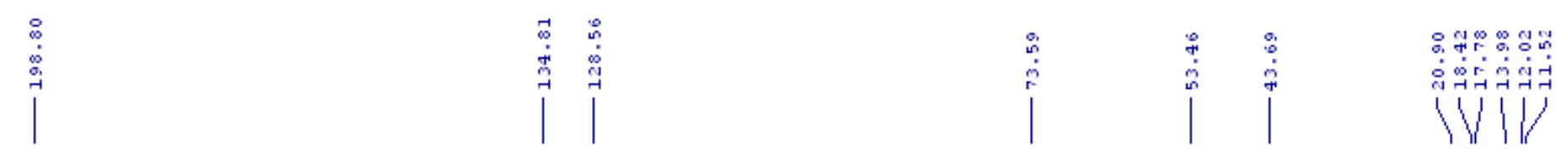

${ }^{13} \mathrm{C}\left\{{ }^{1} \mathrm{H}\right\}\left(100.6 \mathrm{MHz}, \mathrm{CDCl}_{3}\right)$

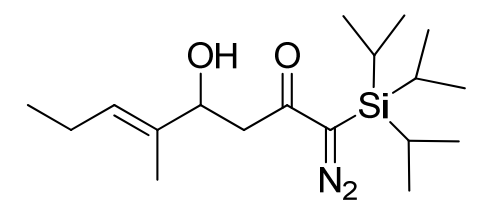

31

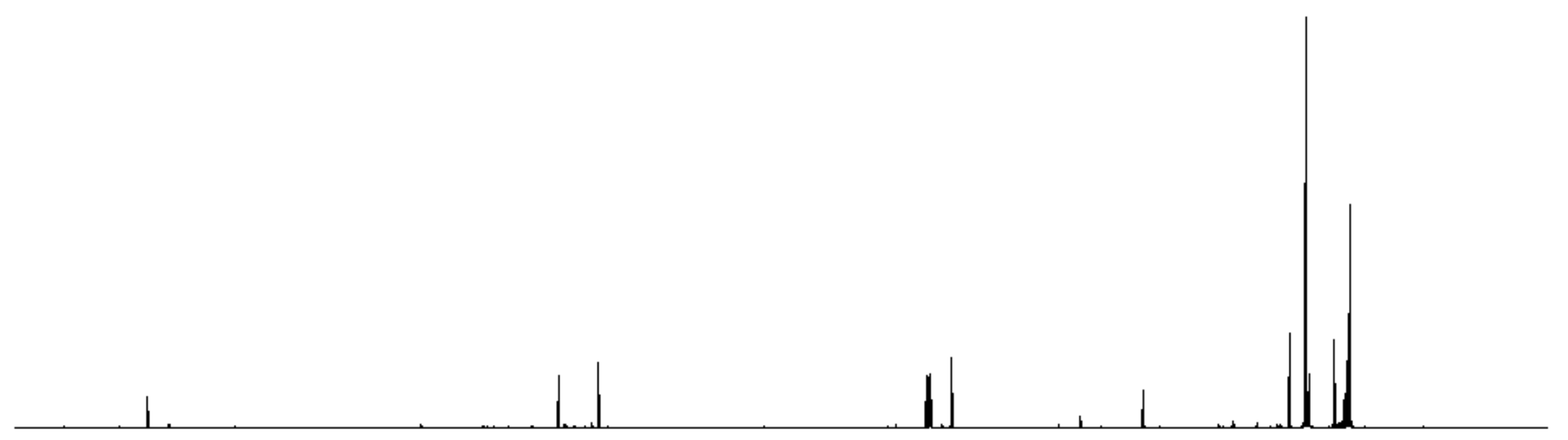

(1) $\begin{array}{llllllllllllllllllllllll}210 & 200 & 190 & 180 & 170 & 160 & 150 & 140 & 130 & 120 & 110 & 100 & 90 & 80 & 70 & 60 & 50 & 40 & 30 & 20 & 10 & 0 & \text { ppm }\end{array}$ 
${ }^{1} \mathrm{H}\left(200 \mathrm{MHz}, \mathrm{CDCl}_{3}\right)$

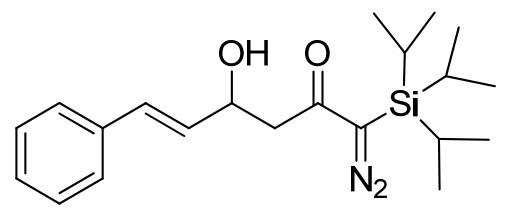

$3 m$

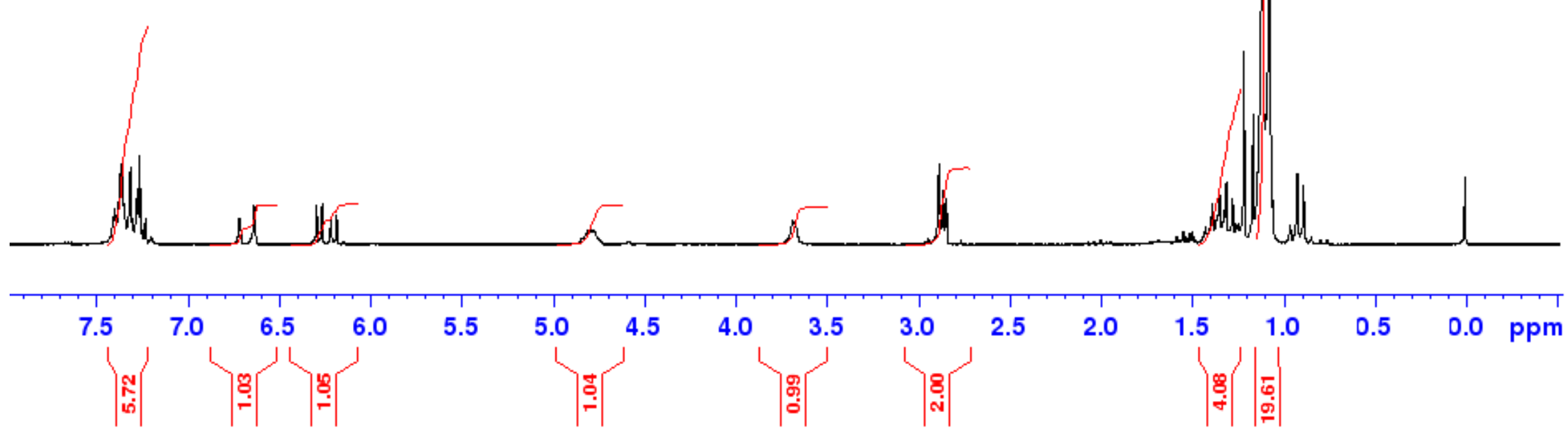




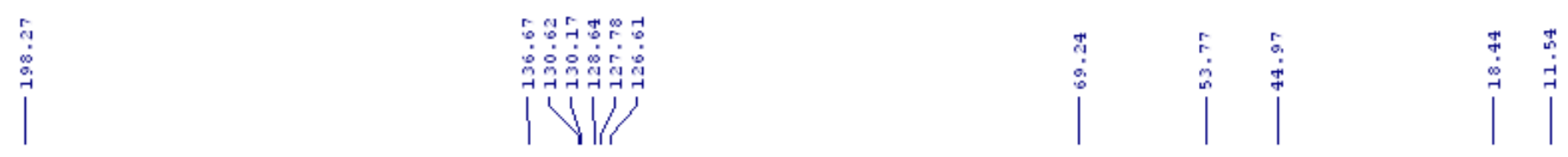

${ }^{13} \mathrm{C}\left\{{ }^{1} \mathrm{H}\right\}\left(100.6 \mathrm{MHz}, \mathrm{CDCl}_{3}\right)$

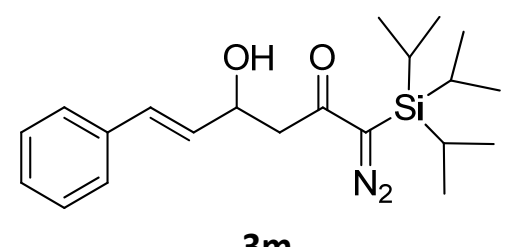

$3 m$

Some degradation occurred during NMR analysis, due to unstability of the product.

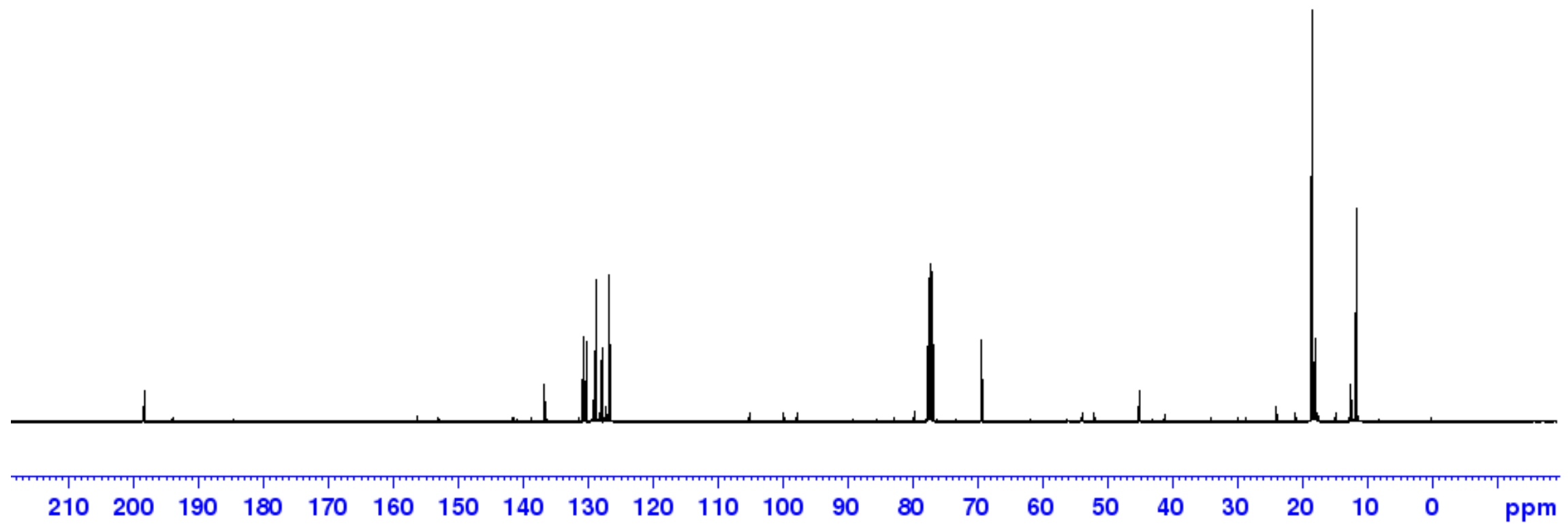




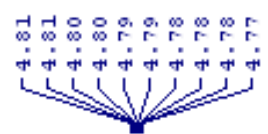

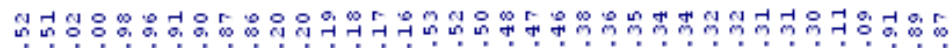

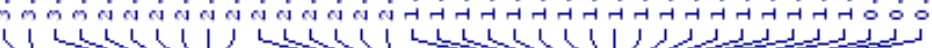

${ }^{1} \mathrm{H}\left(400 \mathrm{MHz}, \mathrm{CDCl}_{3}\right)$

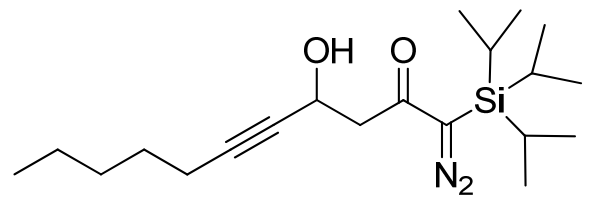

$3 n$

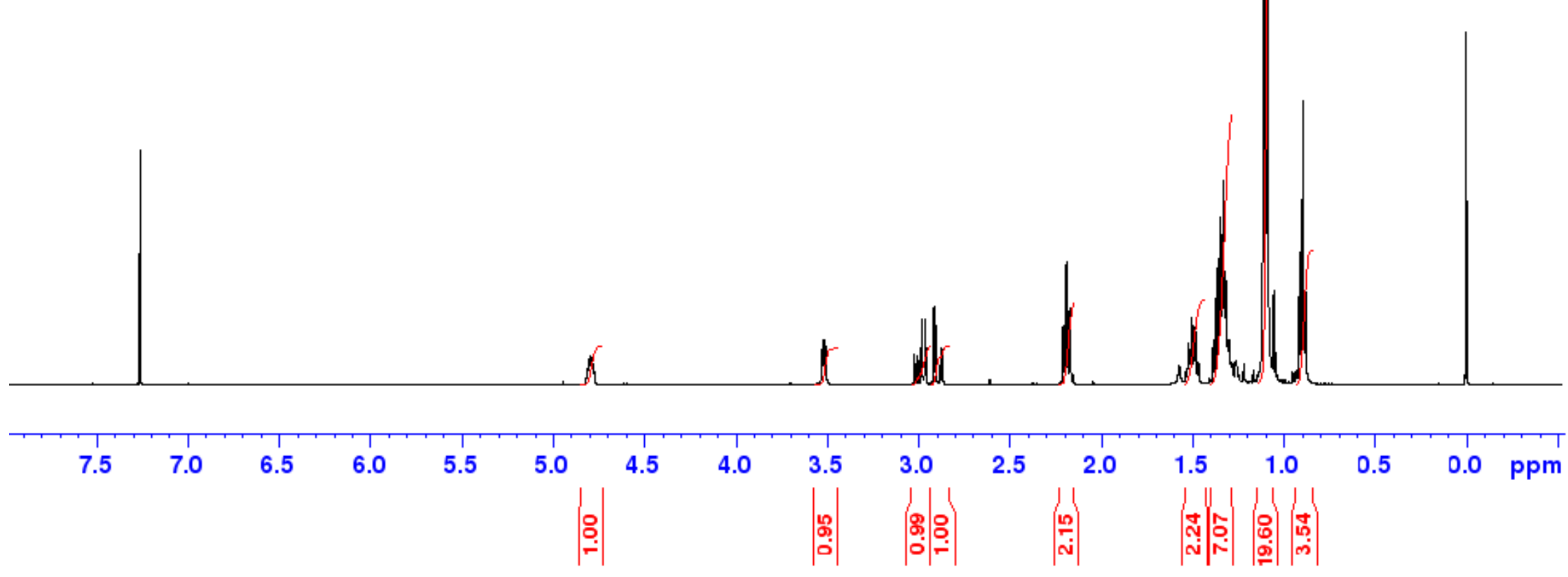




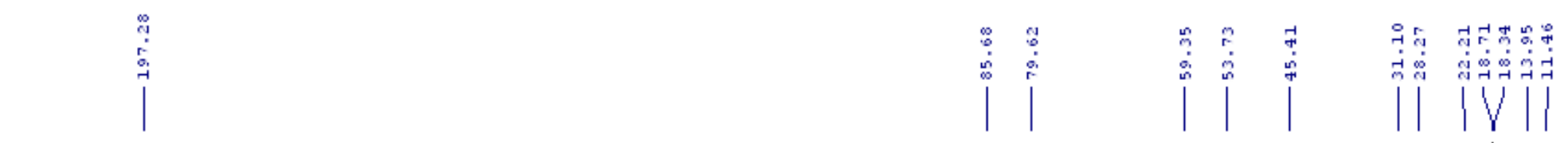

${ }^{13} \mathrm{C}\left\{{ }^{1} \mathrm{H}\right\}\left(100.6 \mathrm{MHz}, \mathrm{CDCl}_{3}\right)$

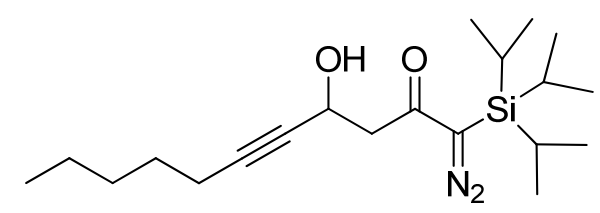

$3 n$

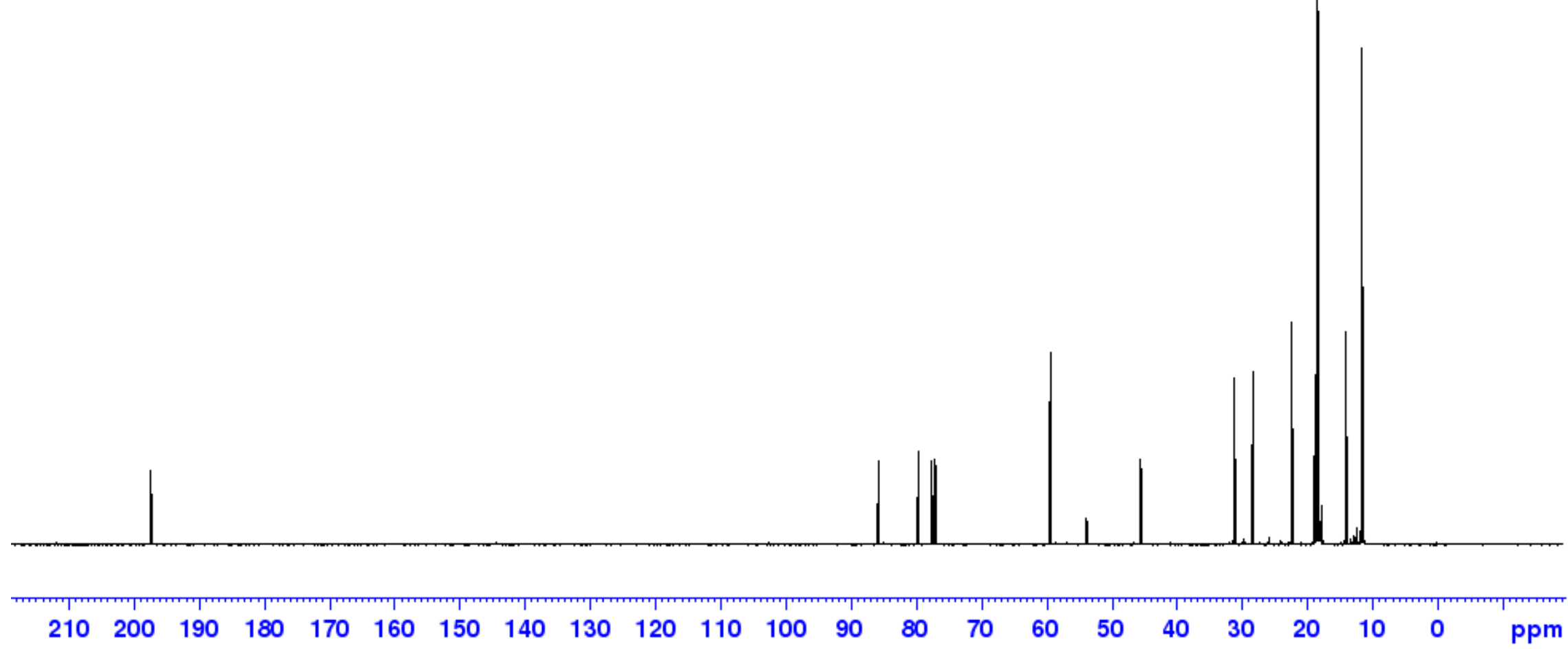




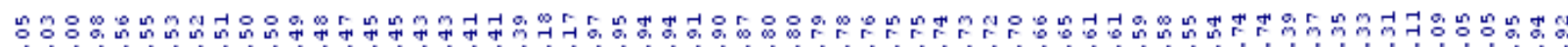
nि L

${ }^{1} \mathrm{H}\left(400 \mathrm{MHz}, \mathrm{CDCl}_{3}\right)$

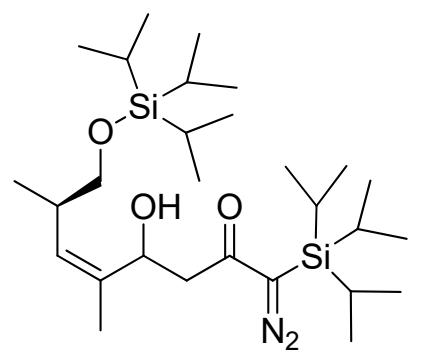

30

(d1+d2)

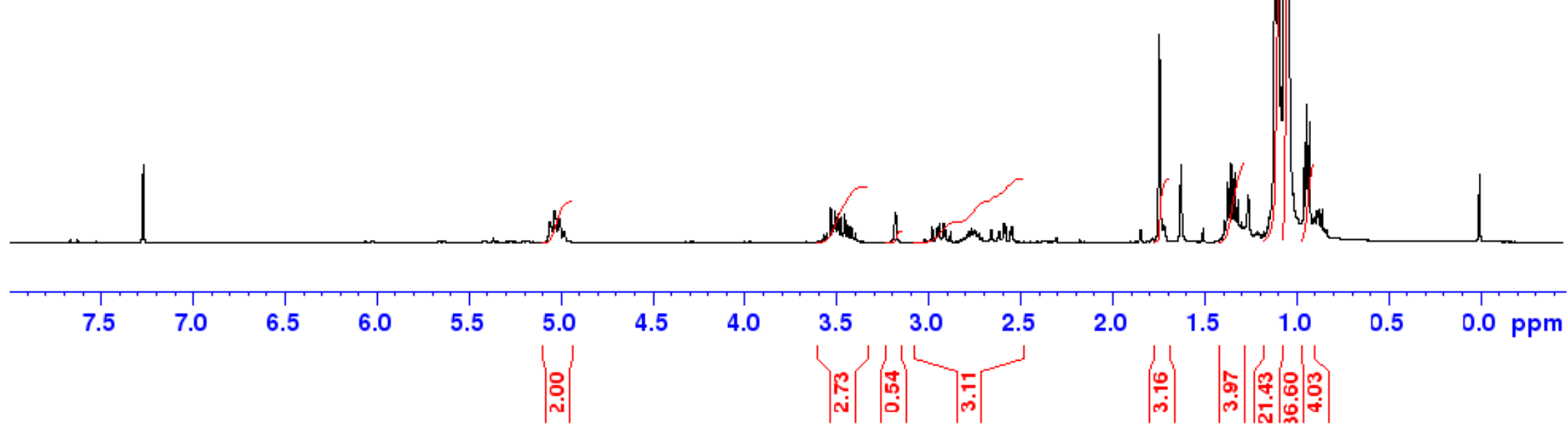




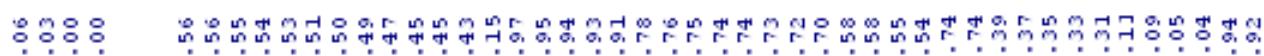

ब1

${ }^{1} \mathrm{H}\left(400 \mathrm{MHz}, \mathrm{CDCl}_{3}\right)$

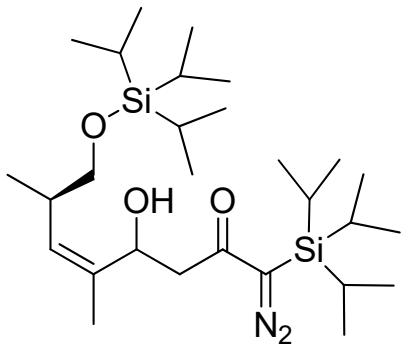

30 (d1)

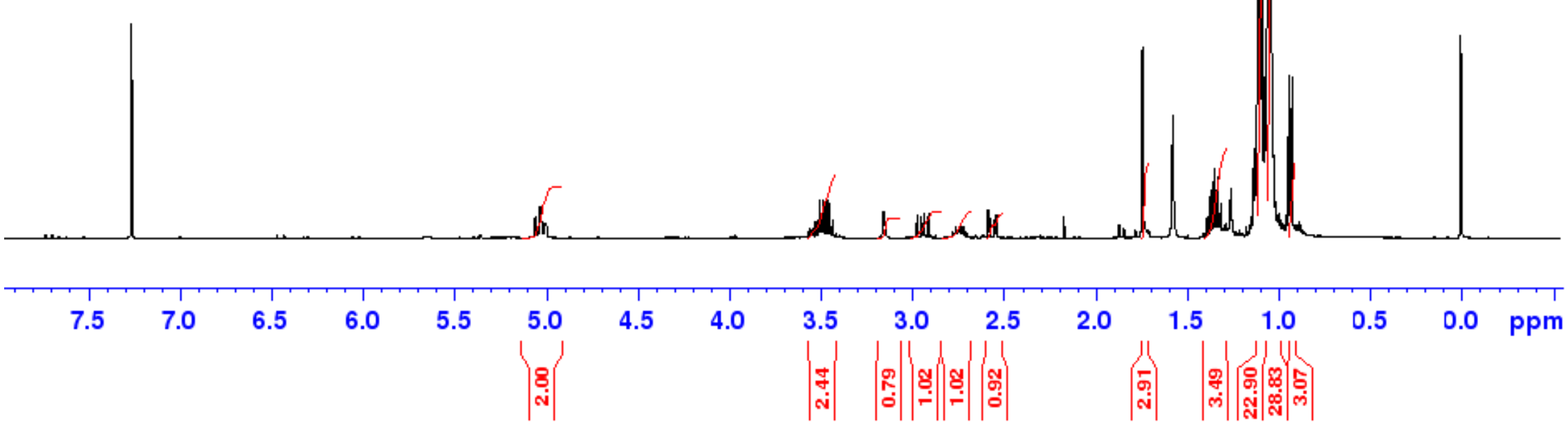




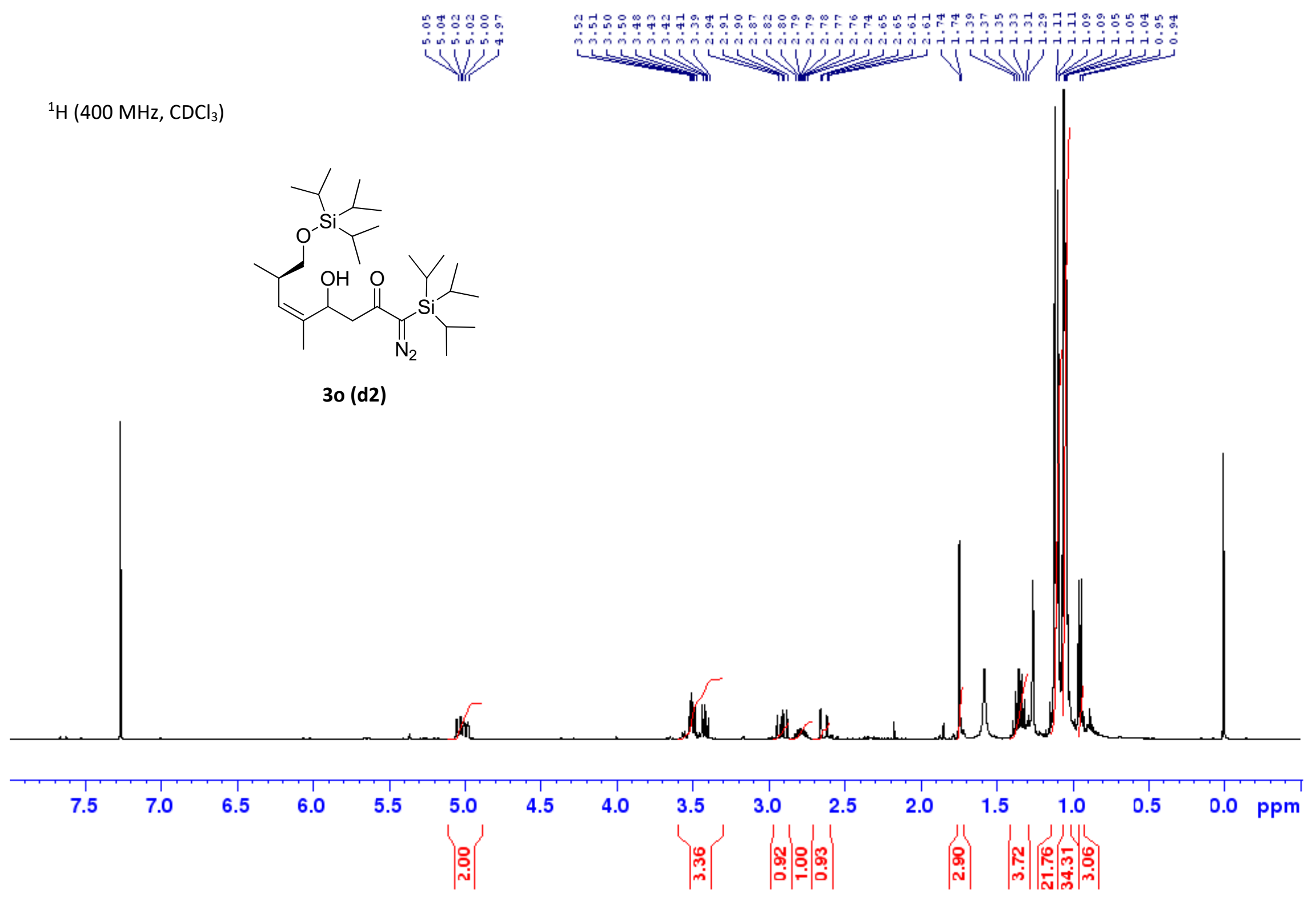


${ }^{13} \mathrm{C}\left\{{ }^{1} \mathrm{H}\right\}\left(100.6 \mathrm{MHz}, \mathrm{CDCl}_{3}\right)$

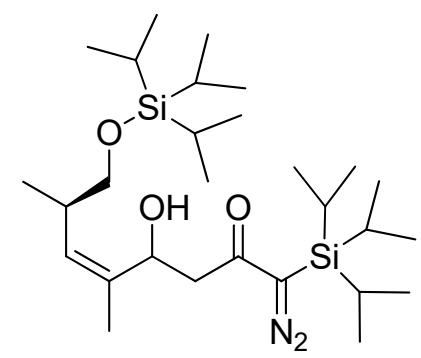

30

(d1+d2)

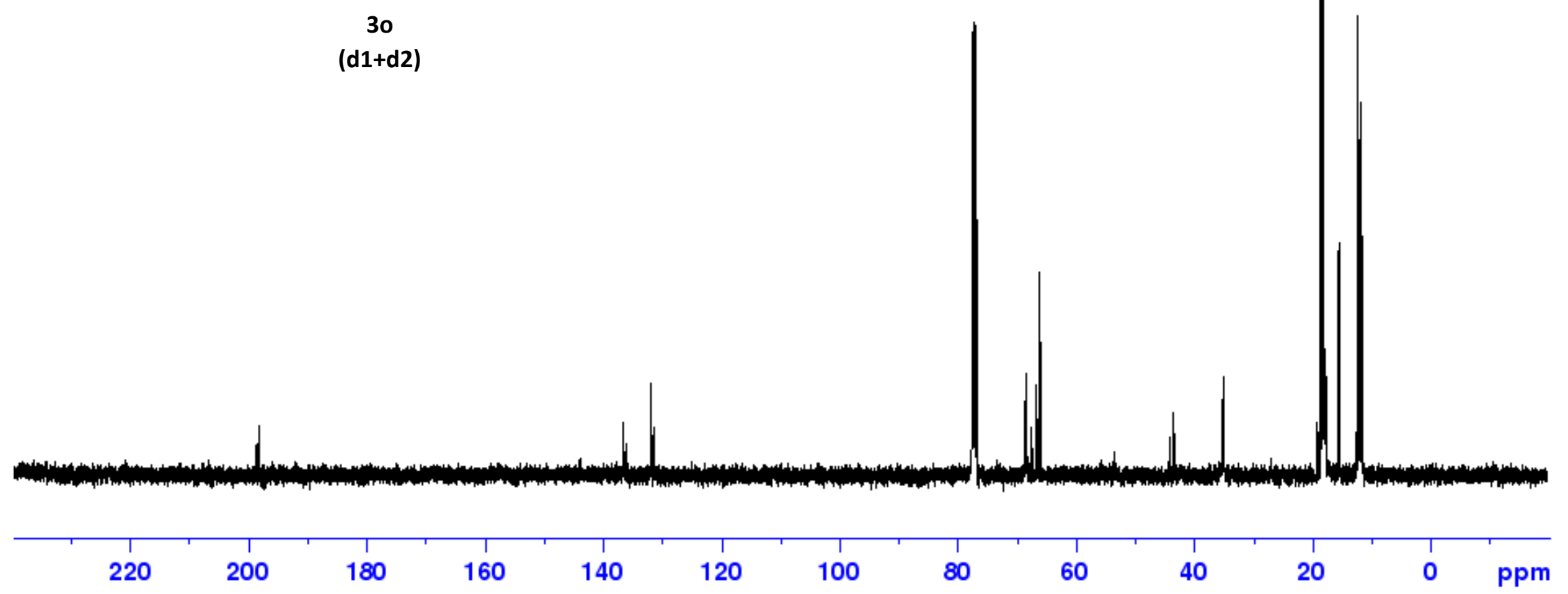


${ }^{1} \mathrm{H}\left(400 \mathrm{MHz}, \mathrm{CDCl}_{3}\right)$

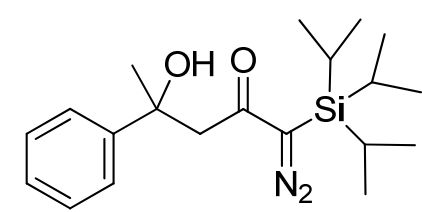

$3 p$

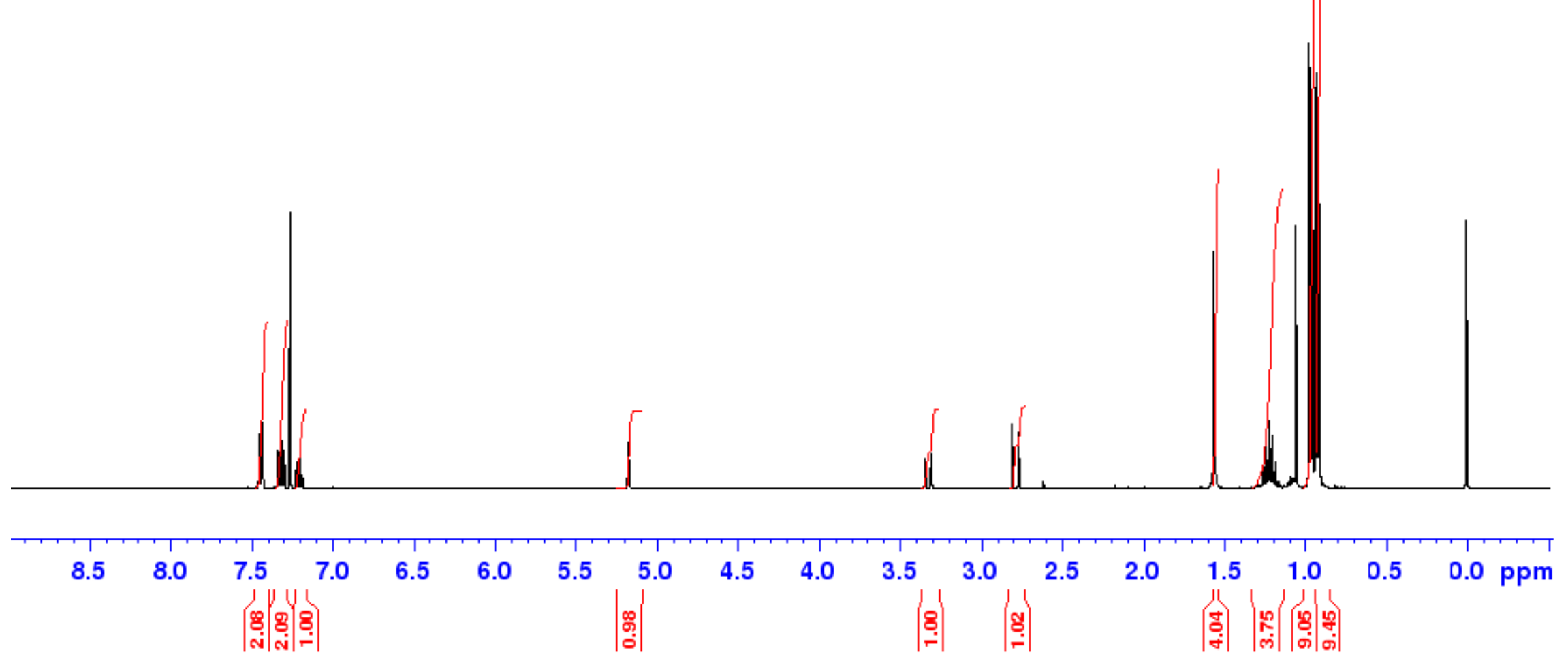




$\mid$

${ }^{13} \mathrm{C}\left\{{ }^{1} \mathrm{H}\right\}\left(100.6 \mathrm{MHz}, \mathrm{CDCl}_{3}\right)$

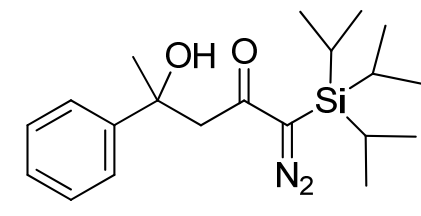

$3 p$

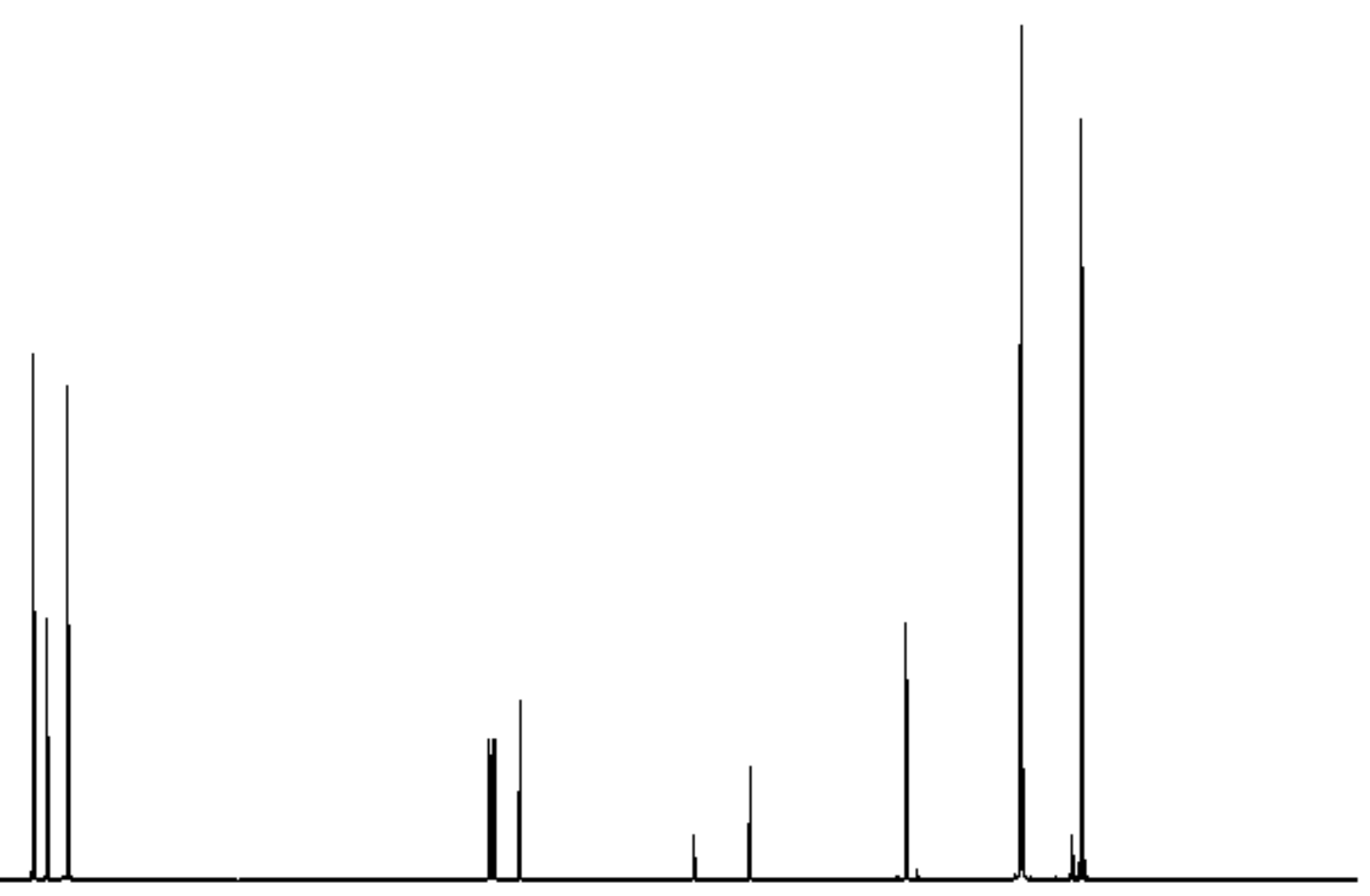

(2. $\begin{array}{lllllllllllllllllllllll}210 & 200 & 190 & 180 & 170 & 160 & 150 & 140 & 130 & 120 & 110 & 100 & 90 & 80 & 70 & 60 & 50 & 40 & 30 & 20 & 10 & 0 & \text { ppm }\end{array}$ 
${ }^{1} \mathrm{H}\left(400 \mathrm{MHz}, \mathrm{CDCl}_{3}\right)$

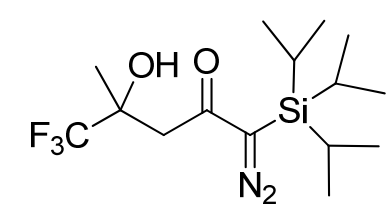

$3 q$

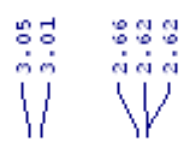

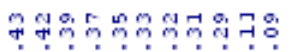

$\stackrel{n}{2}$

$\sqrt{10}$

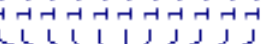

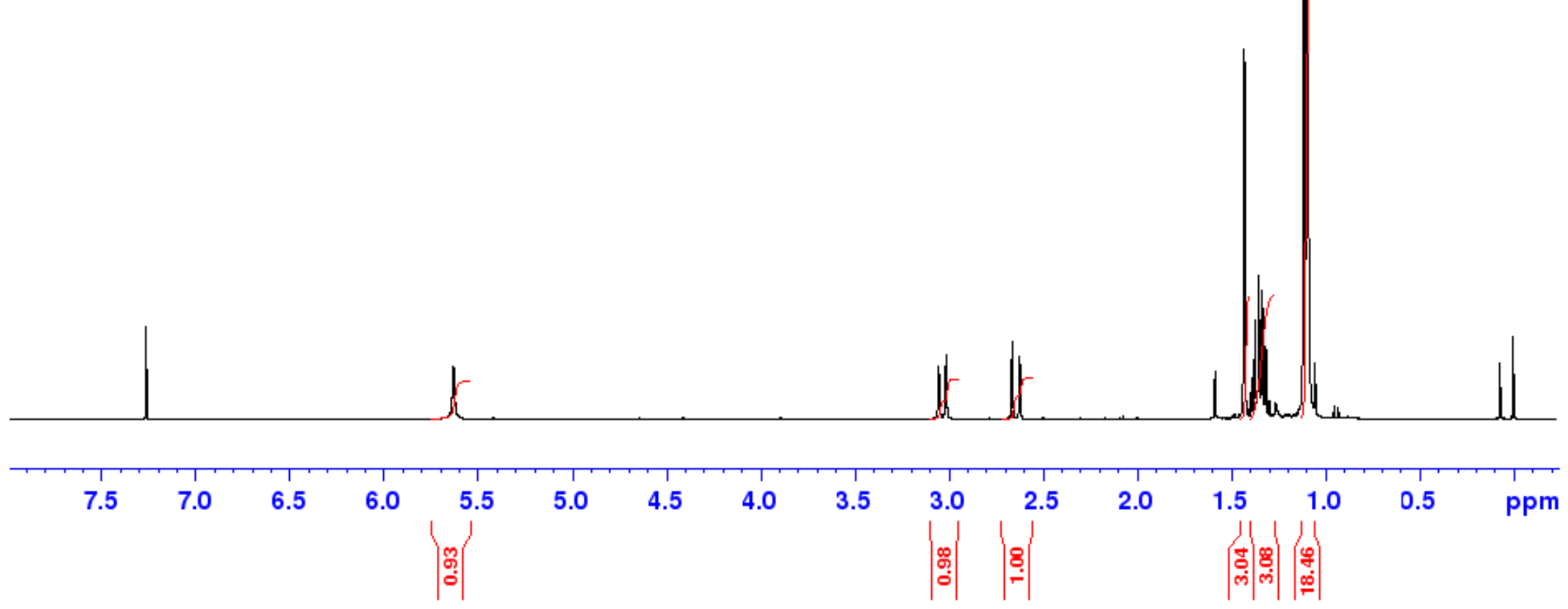



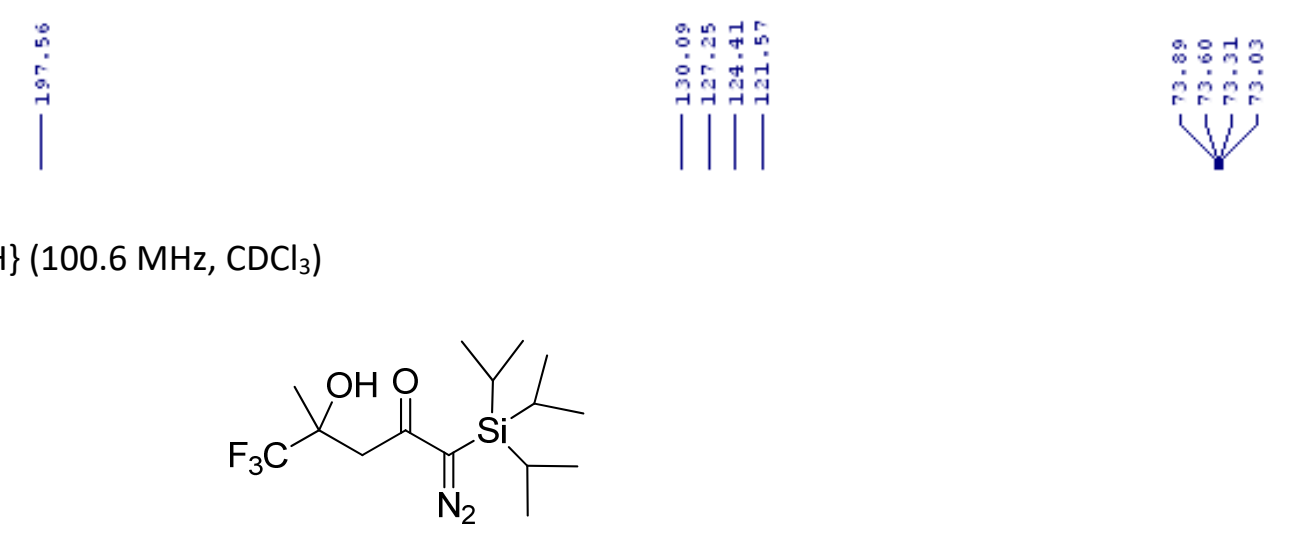

|

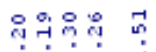

걱

${ }^{13} \mathrm{C}\left\{{ }^{1} \mathrm{H}\right\}\left(100.6 \mathrm{MHz}, \mathrm{CDCl}_{3}\right)$

$3 q$

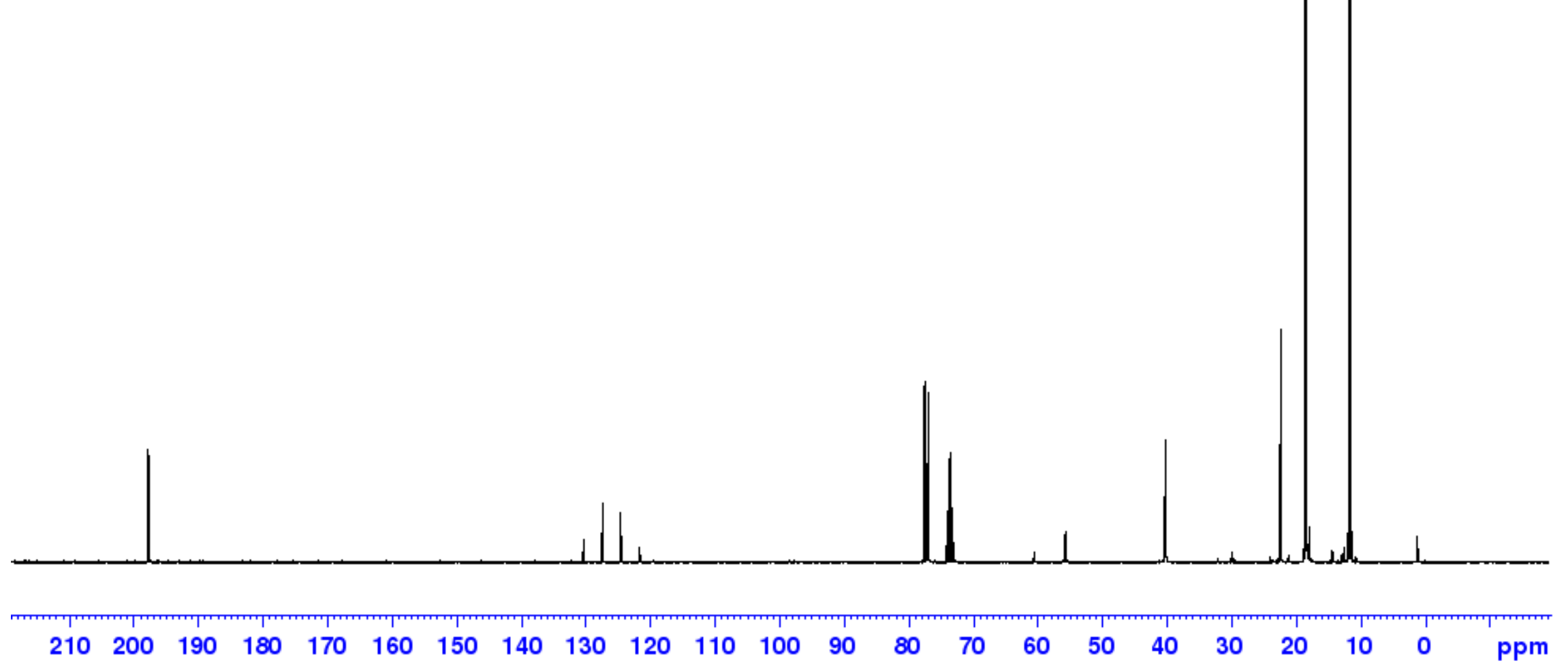


${ }^{19} \mathrm{~F}\left(188.3 \mathrm{MHz}, \mathrm{CDCl}_{3}\right)$

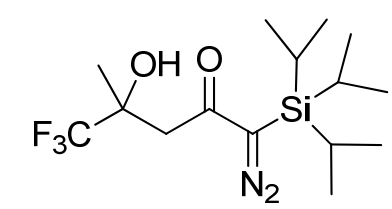

$3 q$

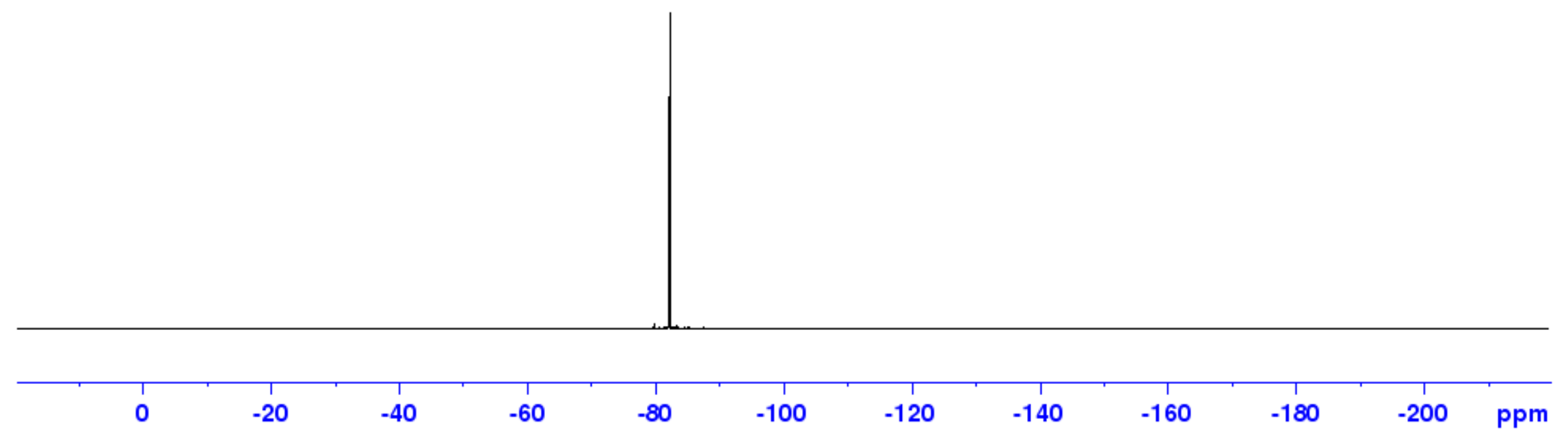




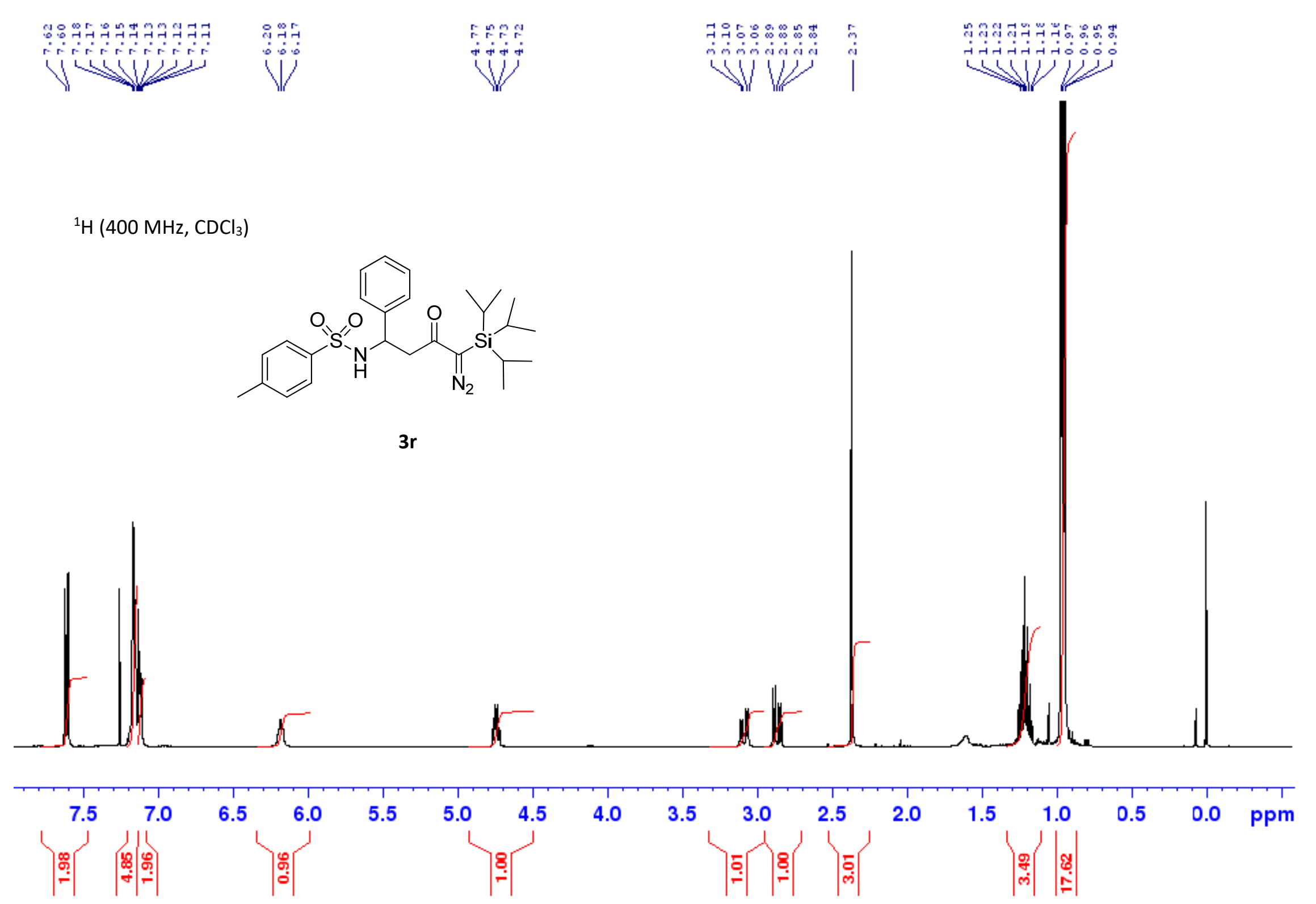




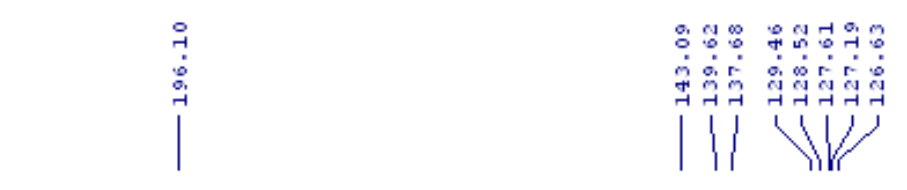

${ }^{13} \mathrm{C}\left\{{ }^{1} \mathrm{H}\right\}\left(100.6 \mathrm{MHz}, \mathrm{CDCl}_{3}\right)$

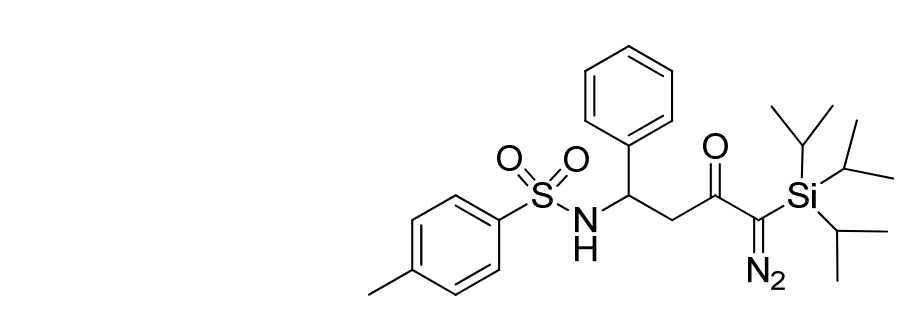

$3 r$
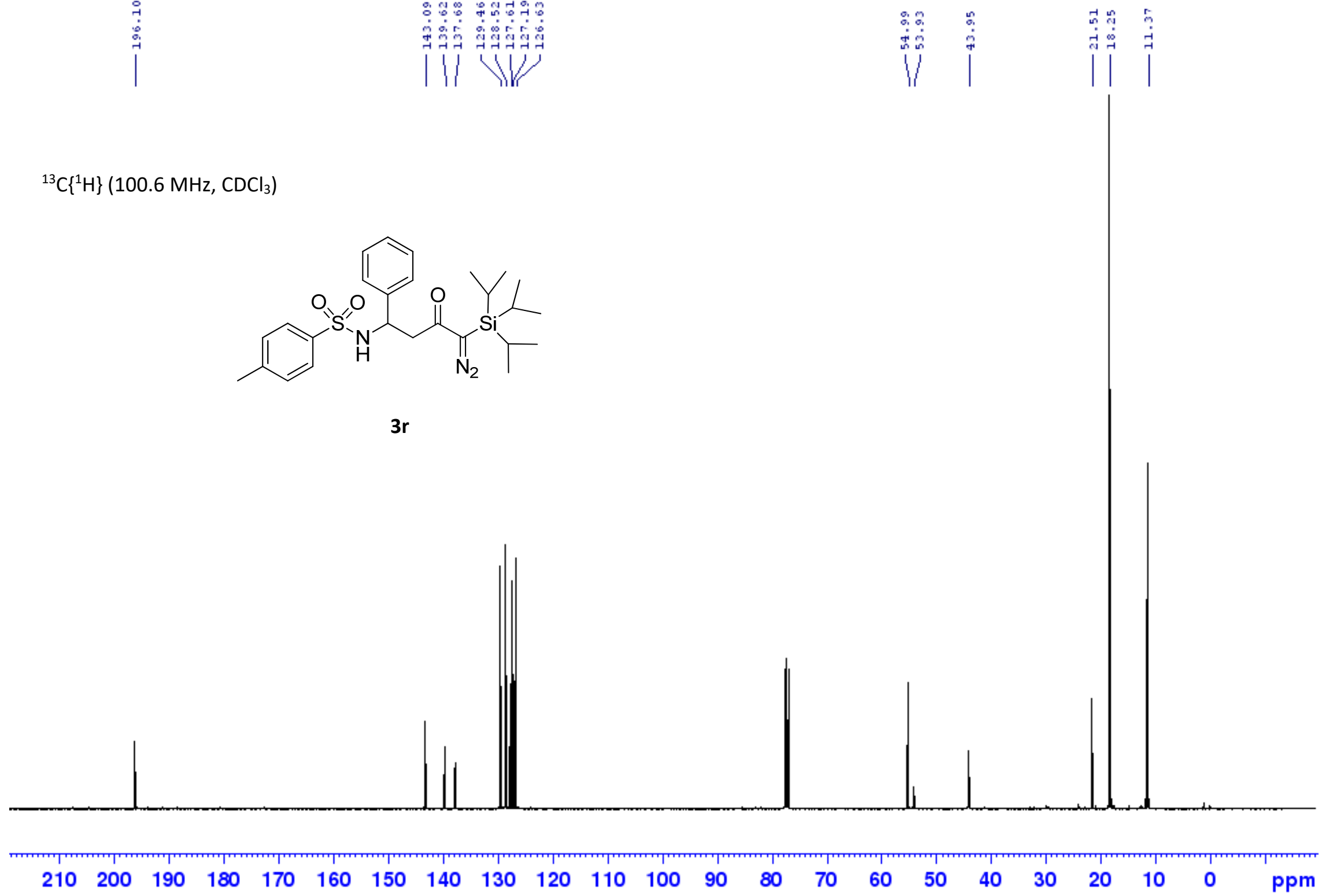
$\mathrm{H}\left(400 \mathrm{MHz}, \mathrm{CDCl}_{3}\right)$

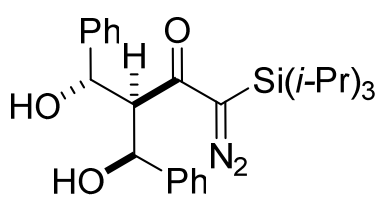

$4 a$

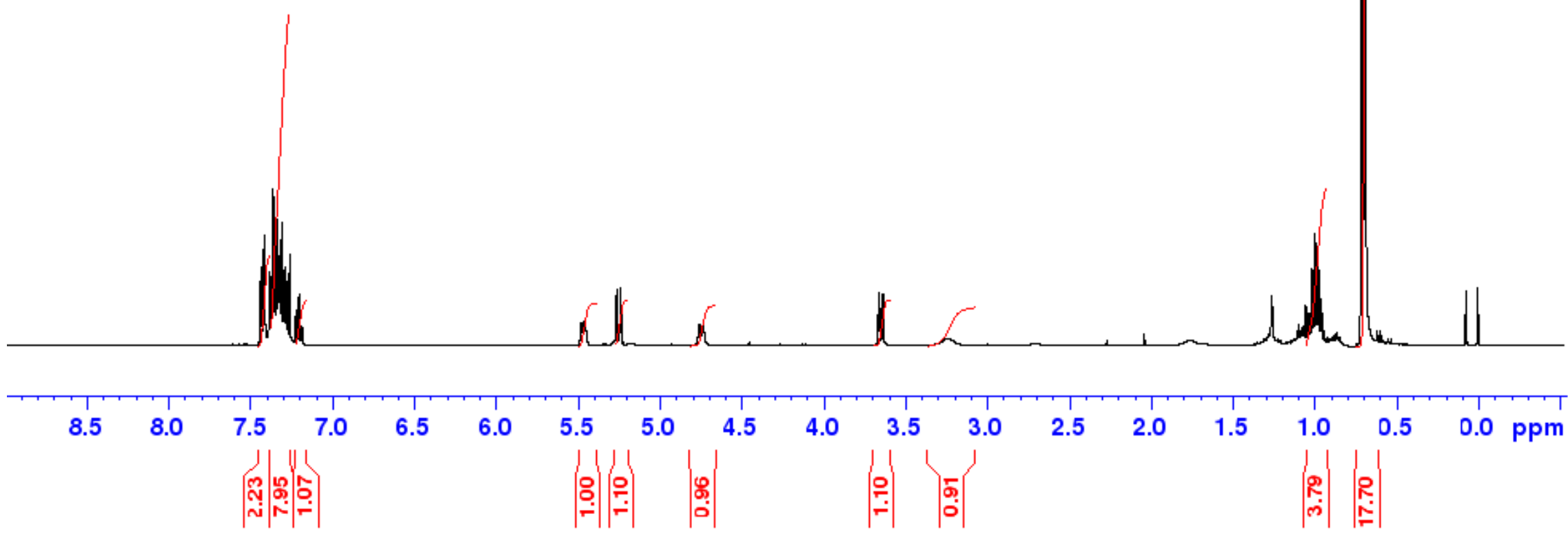


${ }^{13} \mathrm{C}\left\{{ }^{1} \mathrm{H}\right\}\left(100.6 \mathrm{MHz}, \mathrm{CDCl}_{3}\right)$<smiles>N=C([SiH2][Mg])C(=O)[C@@H](C(O)P)[C@@H](O)c1ccccc1</smiles>

$4 a$

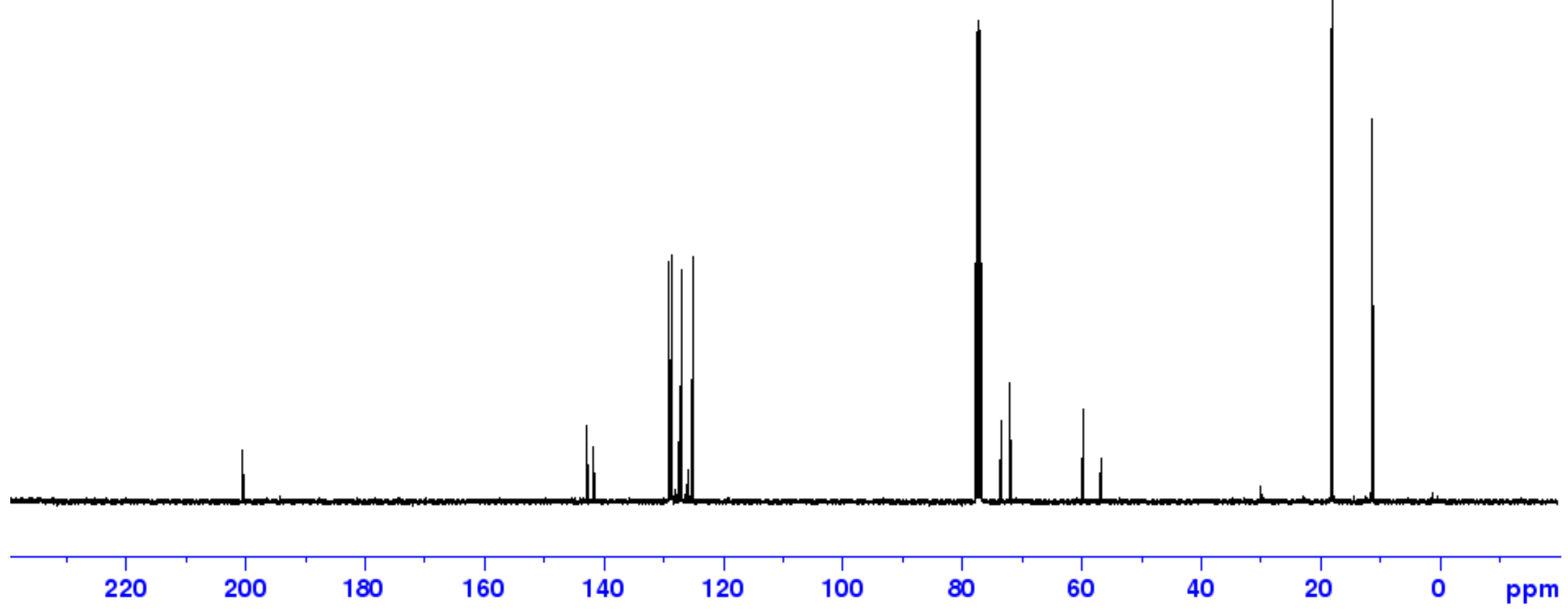




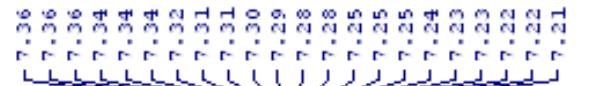

क

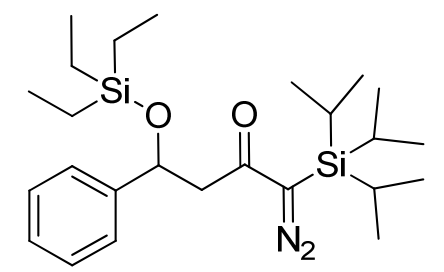

$5 a$

\section{${ }^{1} \mathrm{H}\left(400 \mathrm{MHz}, \mathrm{CDCl}_{3}\right)$}

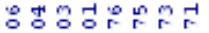

पive

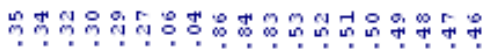

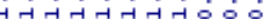

L

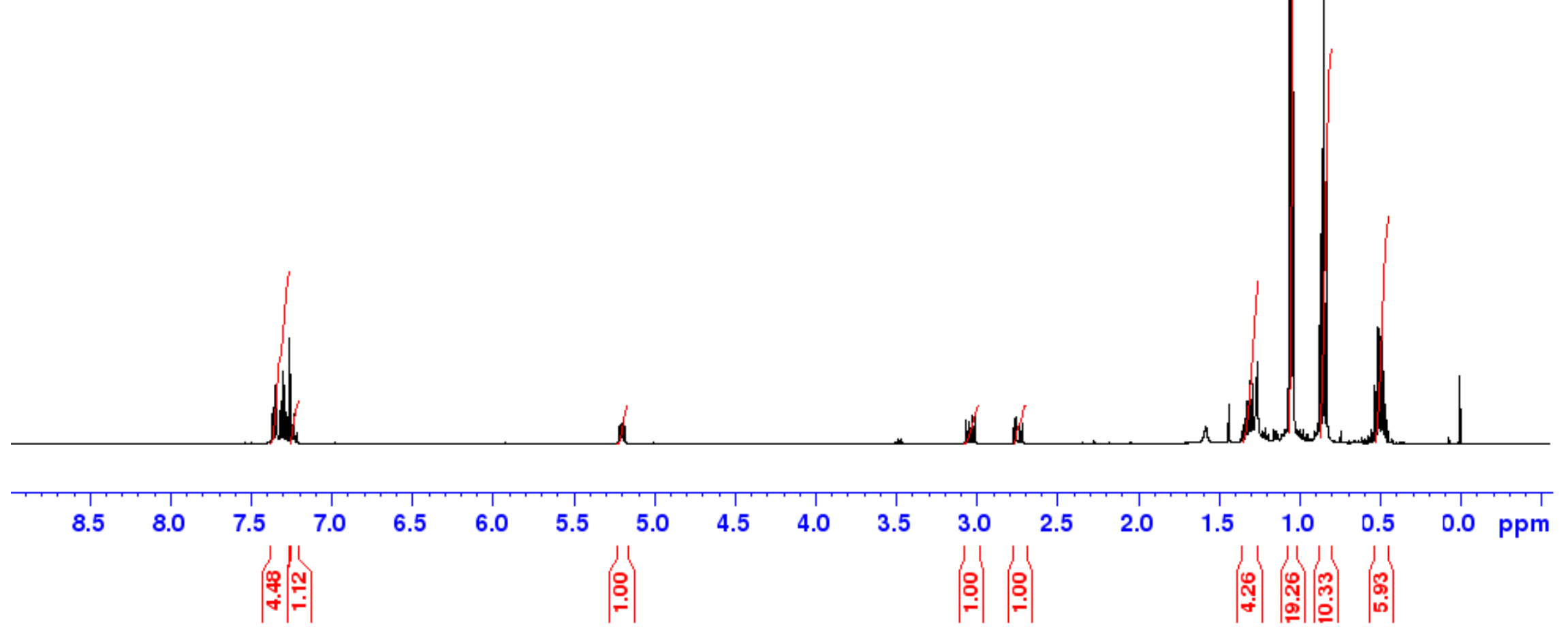




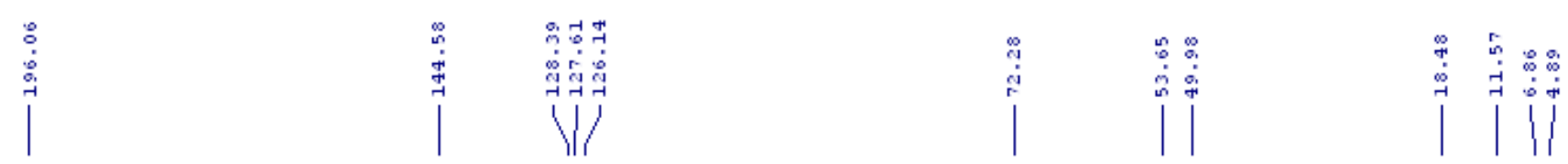

${ }^{13} \mathrm{C}\left\{{ }^{1} \mathrm{H}\right\}\left(100.6 \mathrm{MHz}, \mathrm{CDCl}_{3}\right)$

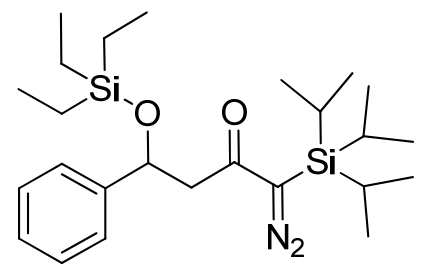

$5 a$

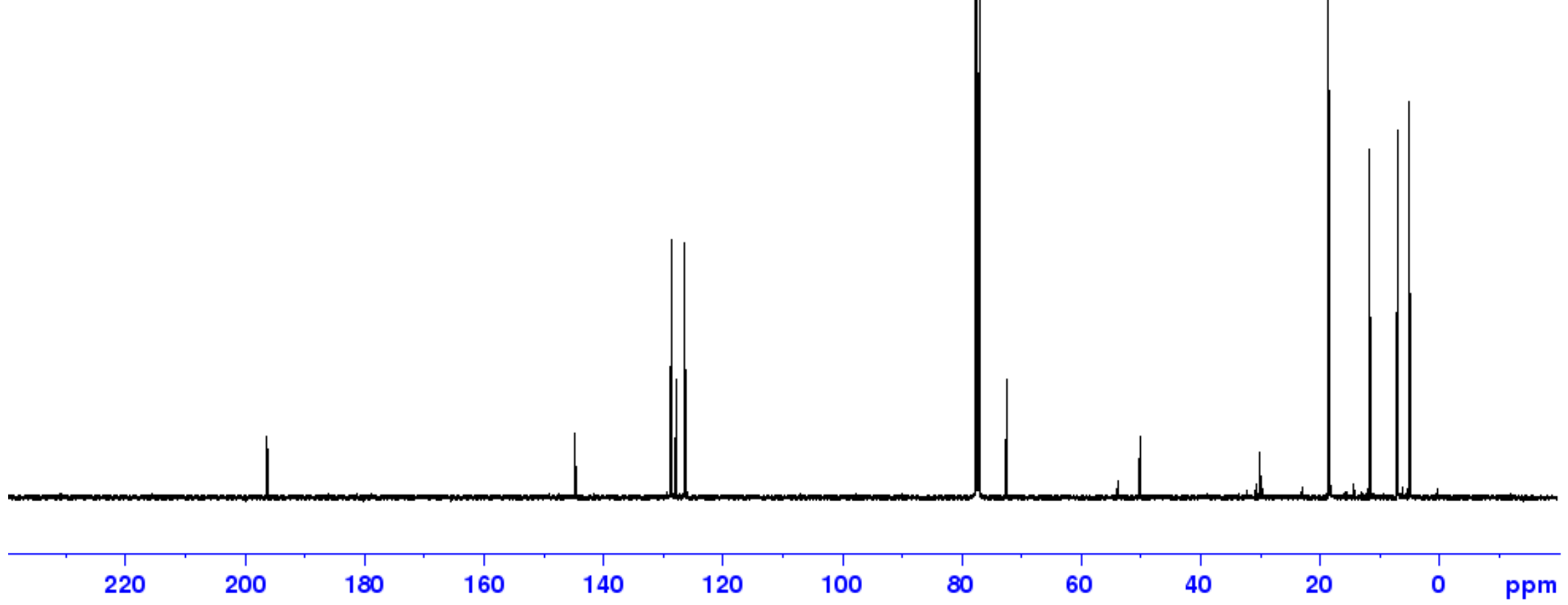




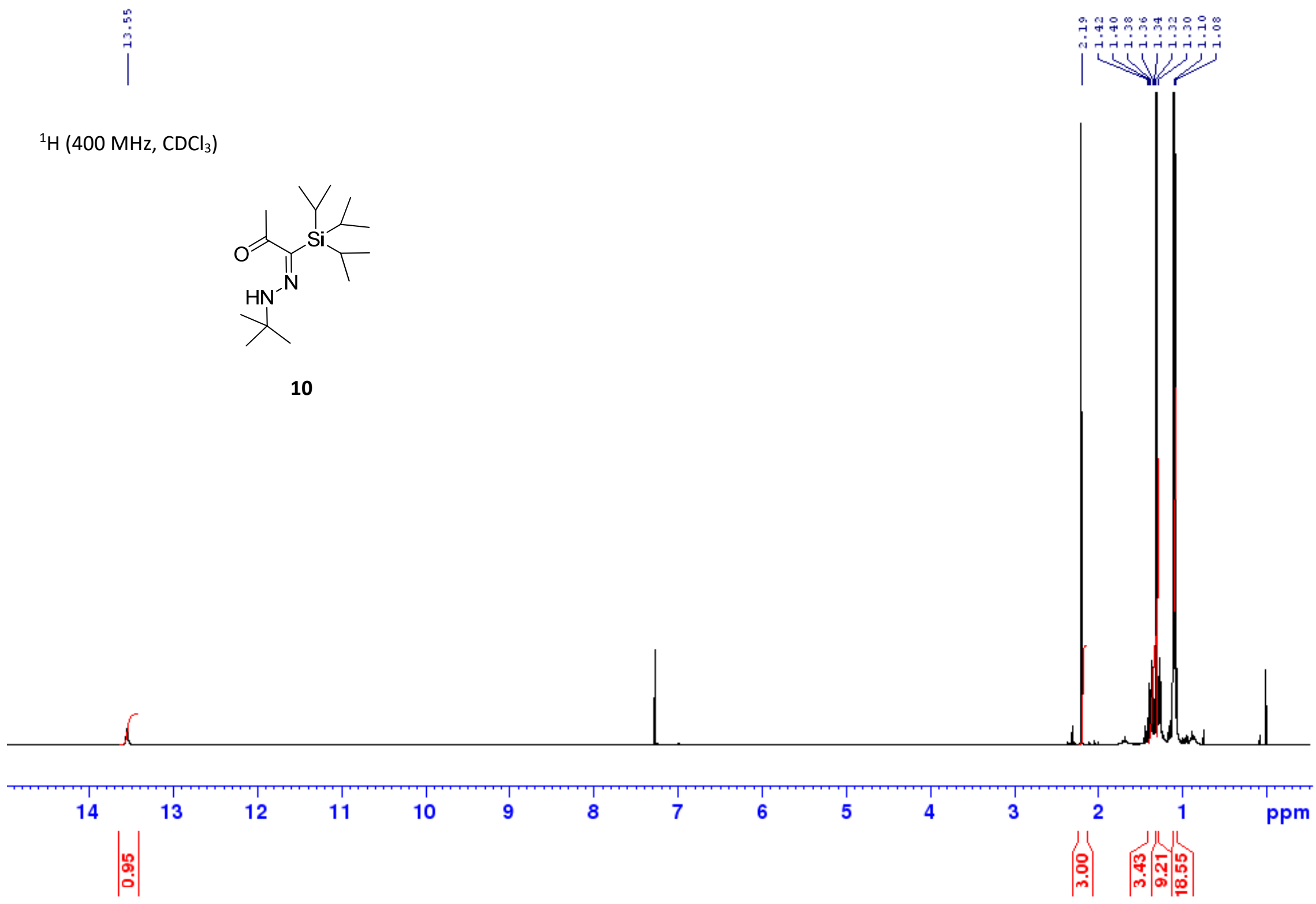




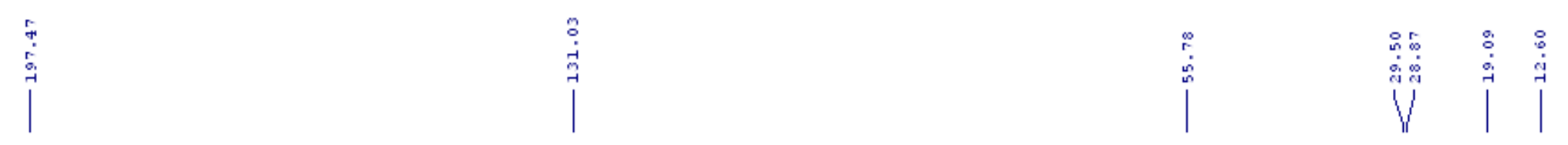

${ }^{13} \mathrm{C}\left\{{ }^{1} \mathrm{H}\right\}\left(100.6 \mathrm{MHz}, \mathrm{CDCl}_{3}\right)$
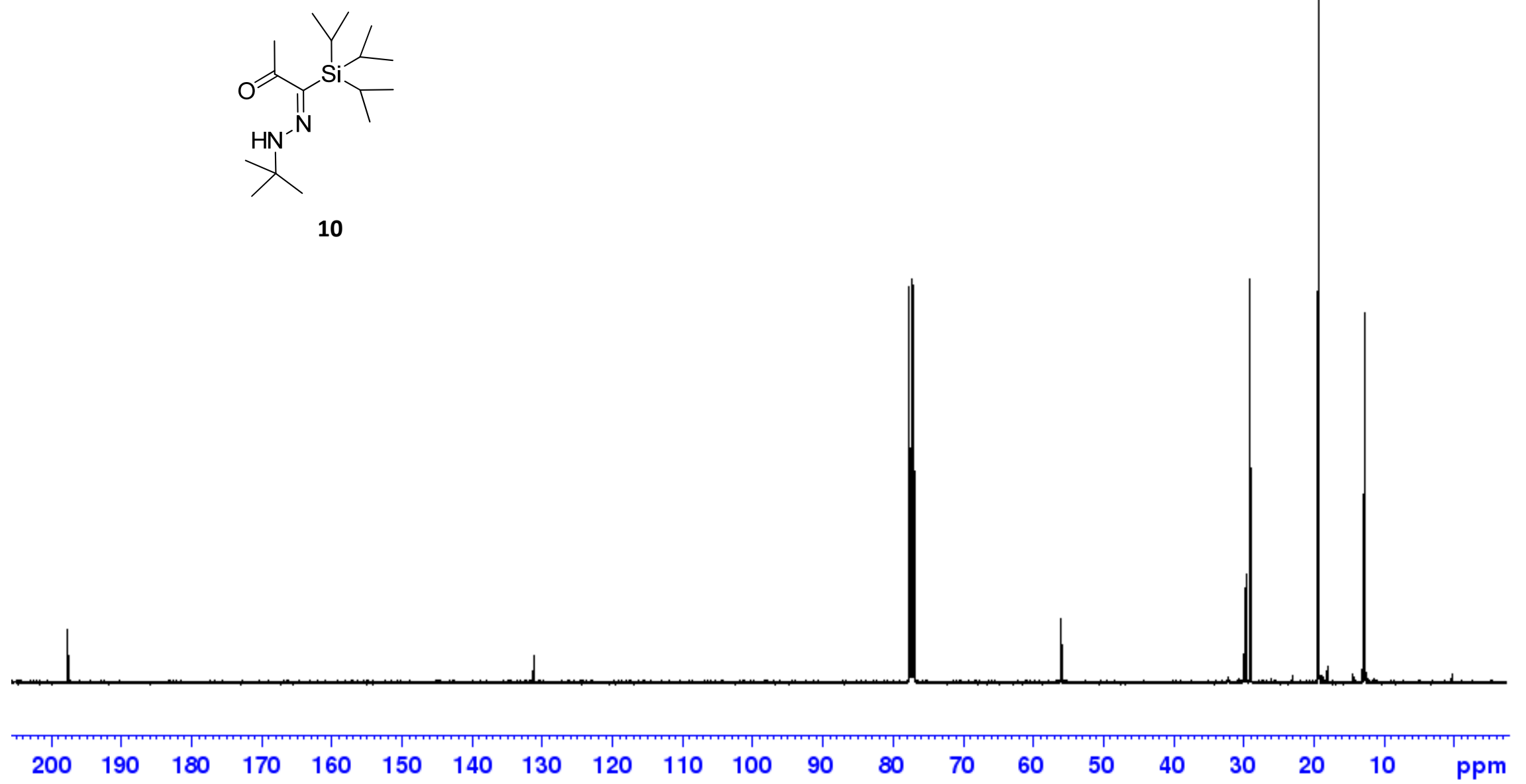
${ }^{1} \mathrm{H}\left(400 \mathrm{MHz}, \mathrm{CDCl}_{3}\right)$

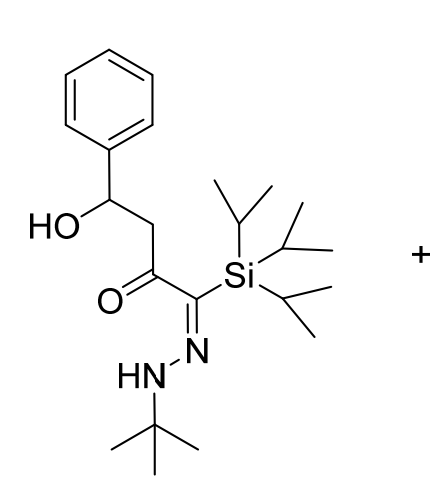

$(E)-11$

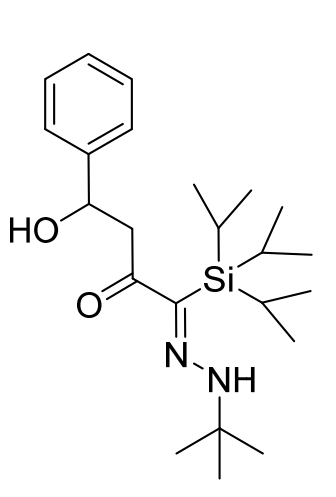

$(Z)-11$

$(E)-11 /(Z)-11=2: 1$

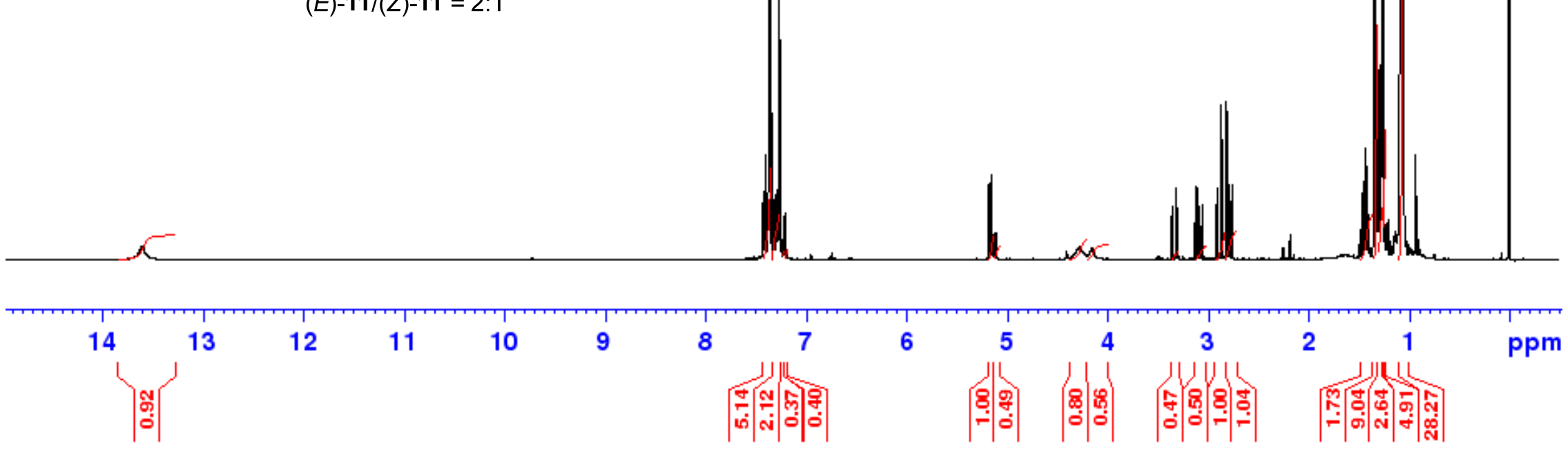




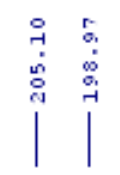

${ }^{13} \mathrm{C}\left\{{ }^{1} \mathrm{H}\right\}\left(100.6 \mathrm{MHz}, \mathrm{CDCl}_{3}\right)$

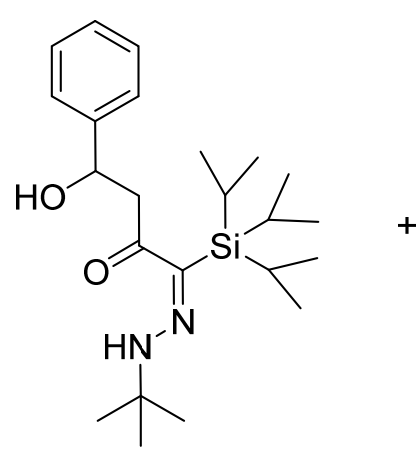

$(E)-11$

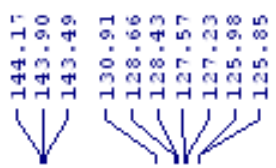

斗出

$\checkmark$ ive

ํㅜㅇ

V

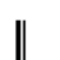

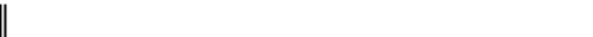

$+$

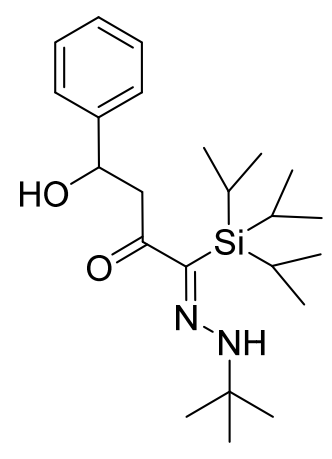

(Z)-11

$(E)-11 /(Z)-11=2: 1$

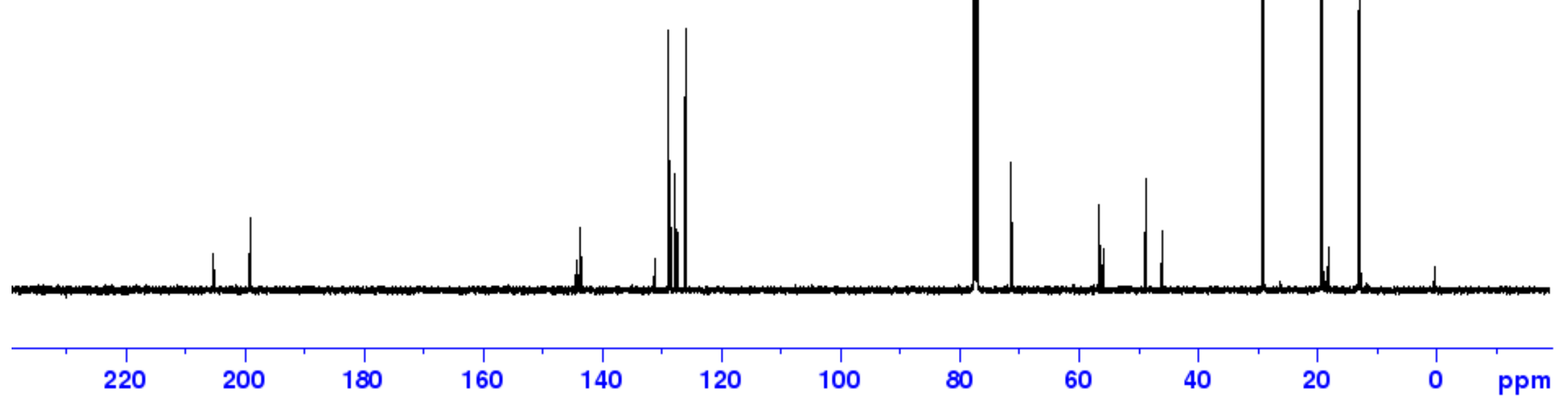




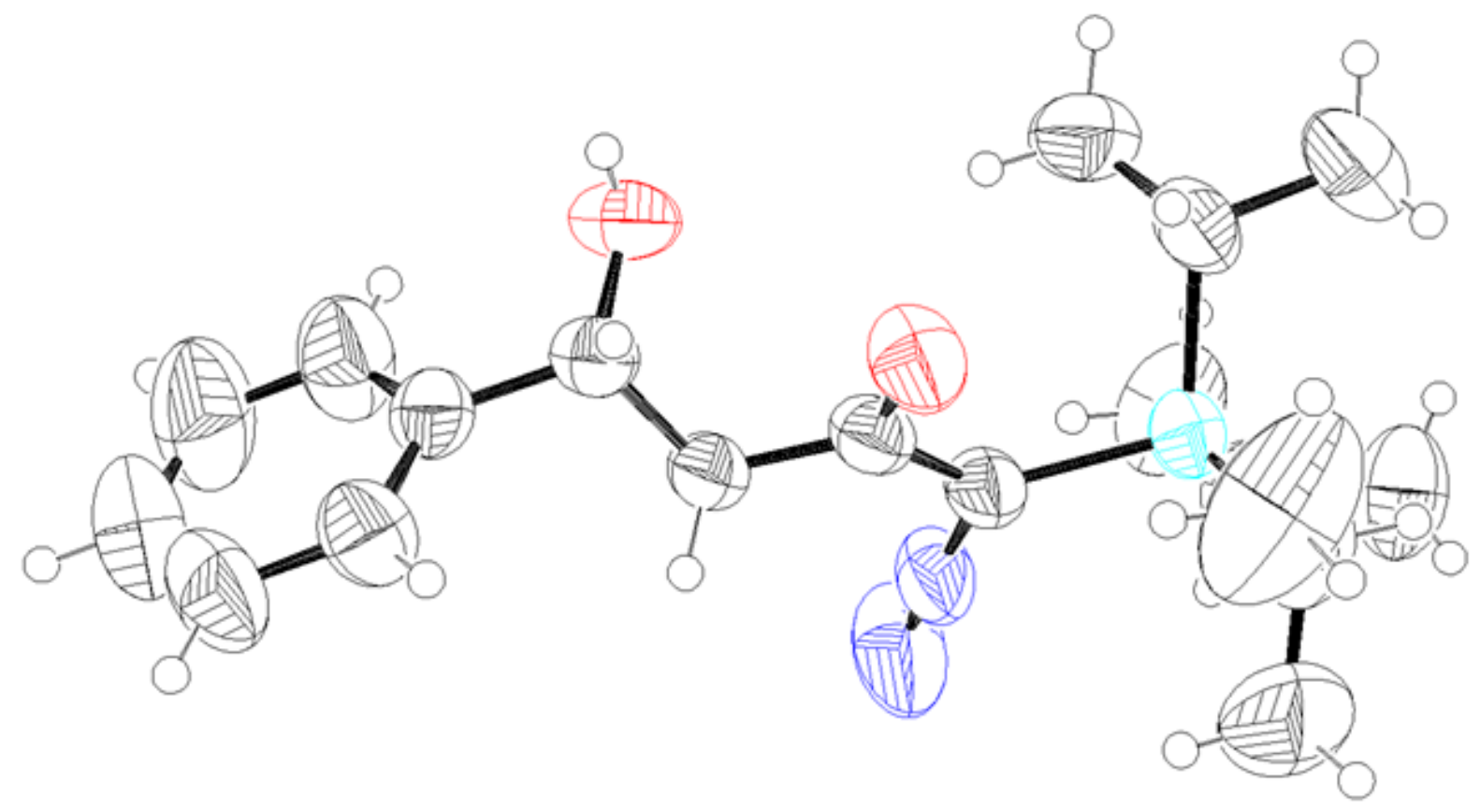

Compound 3a: thermal ellipsoids are shown at the 50\% probability level

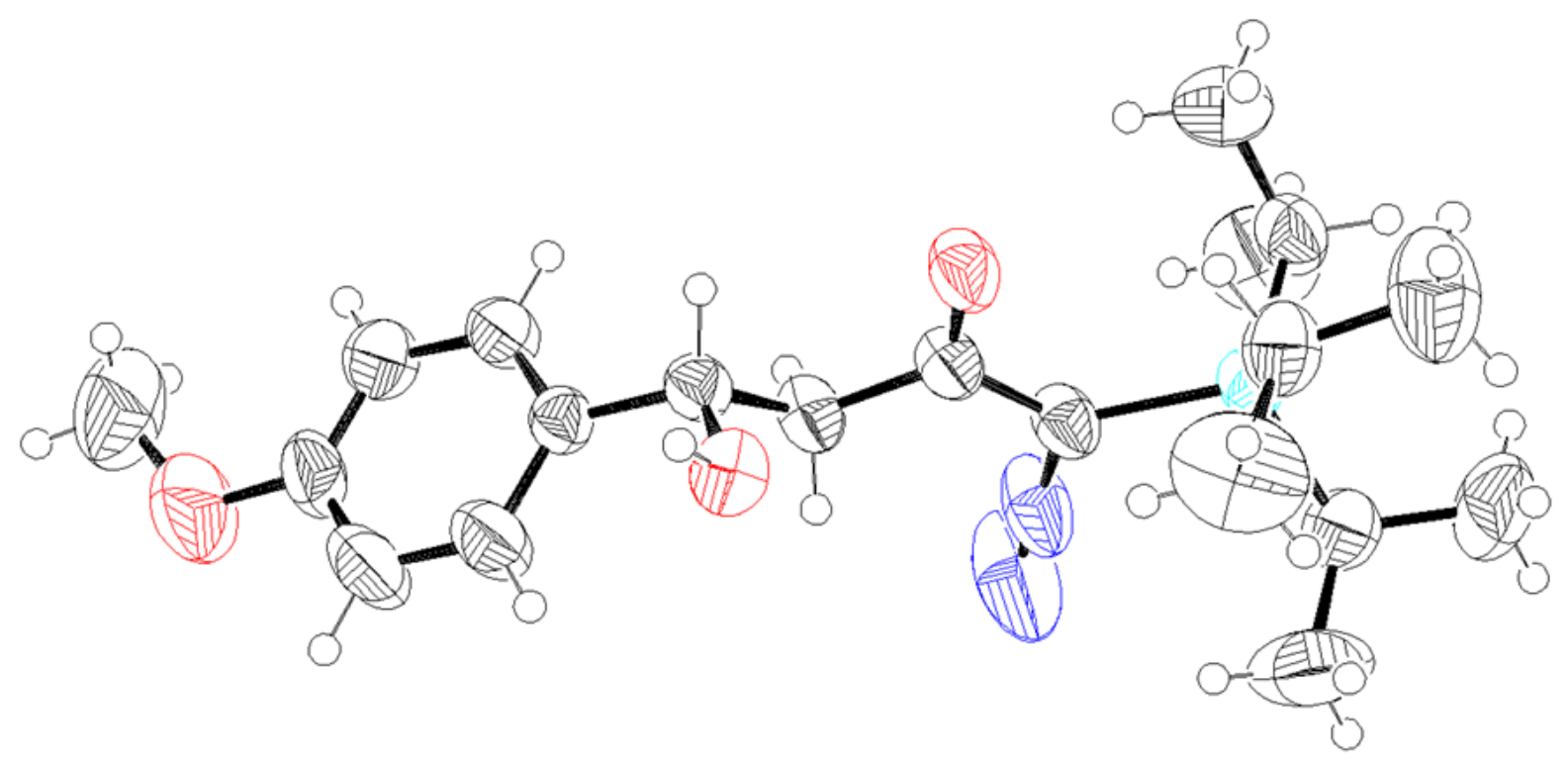

Compound 3b: thermal ellipsoids are shown at the 50\% probability level 


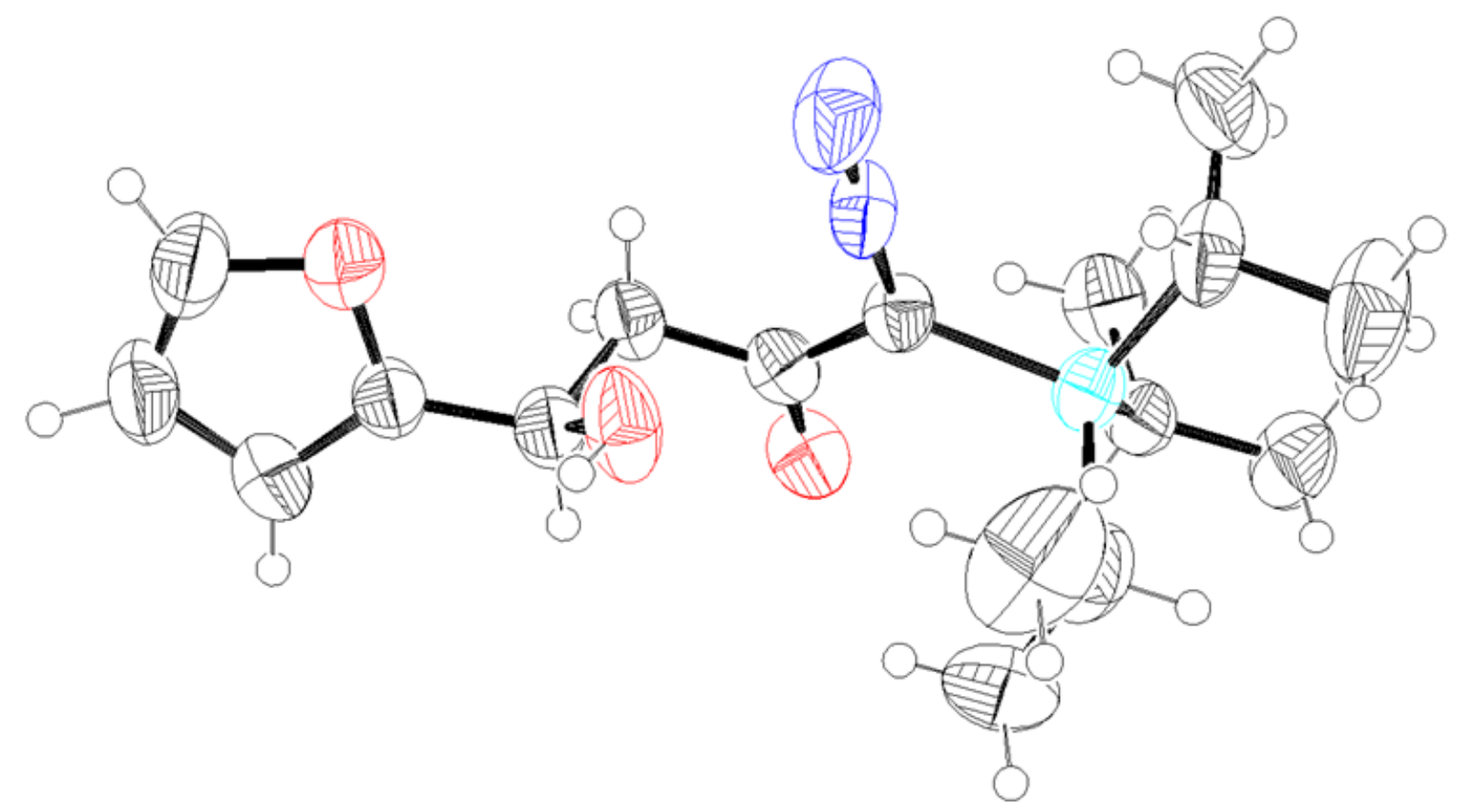

Compound 3e: thermal ellipsoids are shown at the 50\% probability level

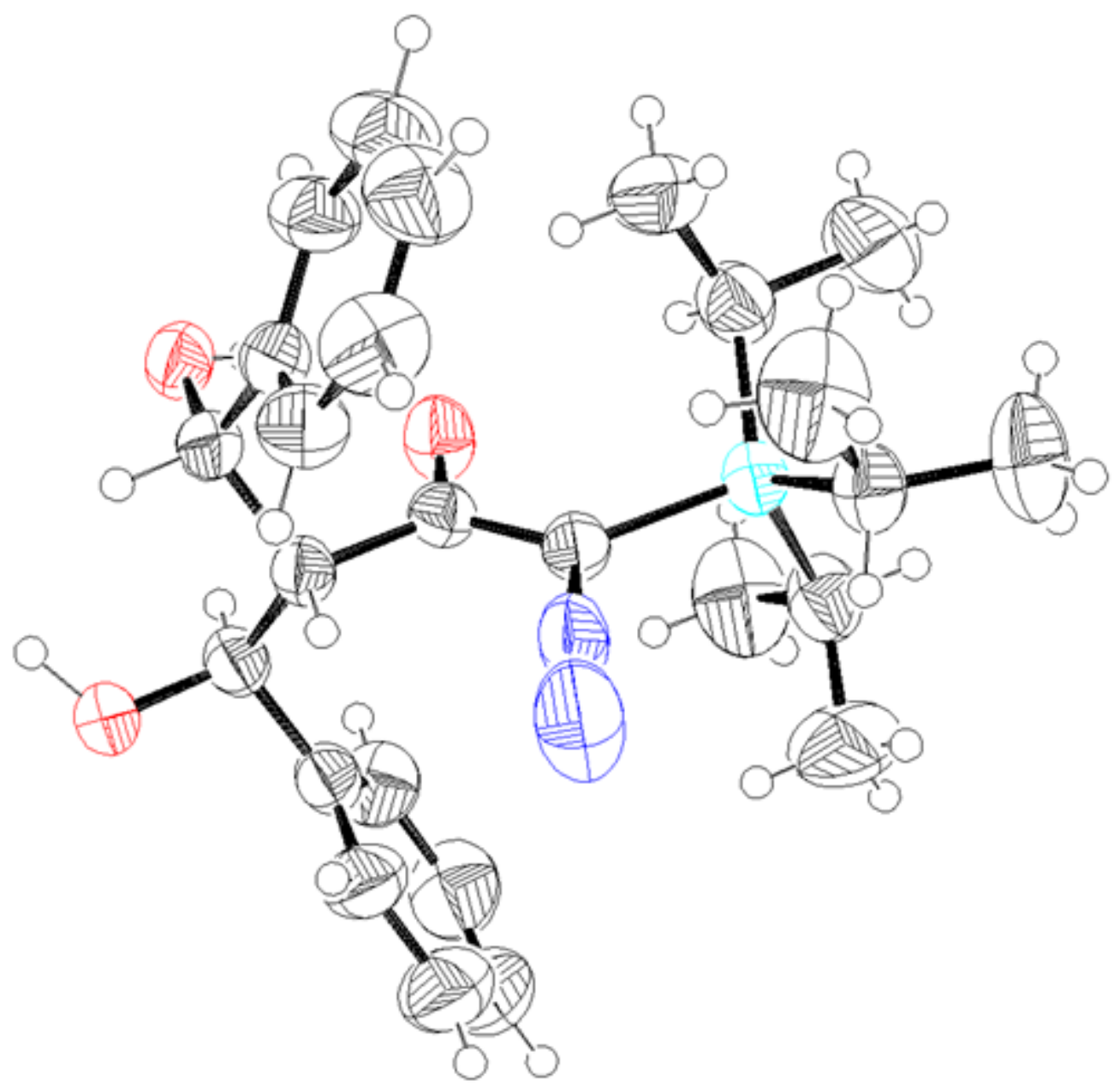

Compound 4a: thermal ellipsoids are shown at the 50\% probability level 


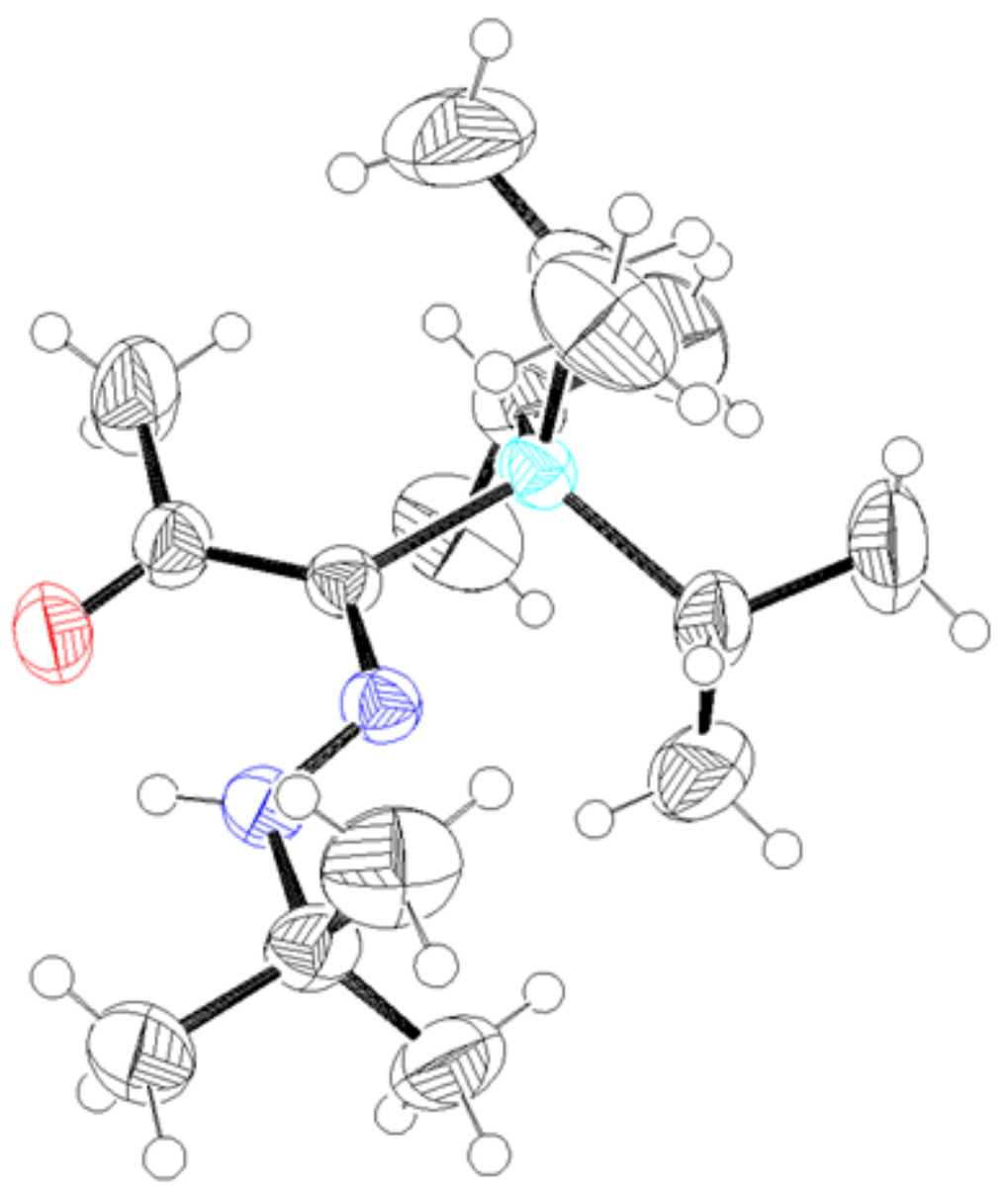

Compound 10: thermal ellipsoids are shown at the 50\% probability level

Table 1. Crystal data and structure refinements of $\mathbf{3 a}, \mathbf{3 b}, \mathbf{3 e}, \mathbf{4 a}$ and $\mathbf{1 0 .}$

\begin{tabular}{|c|c|c|c|c|c|}
\hline Identification code & $3 a$ & $\mathbf{3 b}$ & $3 e$ & $4 a$ & 10 \\
\hline Formula & $\mathrm{C}_{19} \mathrm{H}_{30} \mathrm{~N}_{2} \mathrm{O}_{2} \mathrm{Si}$ & $\mathrm{C}_{20} \mathrm{H}_{32} \mathrm{~N}_{2} \mathrm{O}_{3} \mathrm{Si}$ & $\mathrm{C}_{17} \mathrm{H}_{28} \mathrm{~N}_{2} \mathrm{O}_{3} \mathrm{Si}$ & $\mathrm{C}_{26} \mathrm{H}_{36} \mathrm{~N}_{2} \mathrm{O}_{3} \mathrm{Si}$ & $\mathrm{C}_{16} \mathrm{H}_{34} \mathrm{~N}_{2} \mathrm{OSi}$ \\
\hline Formula mass $\left(\mathrm{g} \cdot \mathrm{mol}^{-1}\right)$ & 346.54 & 376.56 & 336.50 & 452.66 & 298.54 \\
\hline Crystal system & \multicolumn{3}{|c|}{ Monoclinic } & Triclinic & Orthorhombic \\
\hline Space group & $P 2_{1} / n$ & $P 2_{1} / n$ & $P 2_{1} / c$ & $P-1$ & Pbca \\
\hline Unit cell dimensions & & & & & \\
\hline $\mathrm{a}(\AA)=$ & $11.7415(15)$ & $7.903(2)$ & $17.111(2)$ & $7.6367(11)$ & $12.0660(11)$ \\
\hline $\mathrm{b}(\AA)=$ & $12.6469(19)$ & $26.221(6)$ & $9.3240(11)$ & $10.4917(13)$ & $9.5932(8)$ \\
\hline$c(\AA)=$ & $15.0464(17)$ & $11.226(3)$ & $12.2397(12)$ & $17.454(2)$ & $33.550(3)$ \\
\hline$\alpha\left(^{\circ}\right)=$ & 90 & 90 & 90 & 78.193(6) & 90 \\
\hline$\beta\left(^{\circ}\right)=$ & $109.670(7)$ & $107.860(11)$ & $95.717(6)$ & $78.257(6)$ & 90 \\
\hline$\gamma\left({ }^{\circ}\right)=$ & 90 & 90 & 90 & $76.477(6)$ & 90 \\
\hline Volume, Z & $2103.9(5), 4$ & $2214.1(10), 4$ & 1943.0(4), 4 & 1313.0(3), 2 & $3883.5(6), 8$ \\
\hline Calculated density & 1.094 & 1.130 & 1.150 & 1.145 & 1.021 \\
\hline
\end{tabular}




\begin{tabular}{|c|c|c|c|c|c|}
\hline$\left(\right.$ g.cm $\left.{ }^{-3}\right)$ & & & & & \\
\hline $\begin{array}{l}\text { Absorption coefficient } \\
\qquad\left(\mathrm{mm}^{-1}\right)\end{array}$ & 0.124 & 0.126 & 0.136 & 0.117 & 0.121 \\
\hline $\mathrm{F}_{000}$ & 752 & 816 & 728 & 488 & 1328 \\
\hline Crystal size & $0.366 * 0.631 * 0.248$ & $0.429 * 0.280 * 0.208$ & $0.303 * 0.292 * 0.164$ & $0.463 * 0.157 * 0.137$ & $0.734 * 0.410 * 0.121$ \\
\hline $\begin{array}{l}2 \theta \text { range for data } \\
\text { collection }\left(^{\circ}\right)\end{array}$ & $4.3-55.3$ & $4.1-54.3$ & $4.8-55.1$ & $4.0-55.1$ & $4.8-55.4$ \\
\hline $\begin{array}{l}(\mathrm{hkl})_{\min } \\
(\mathrm{hkl})_{\max }\end{array}$ & 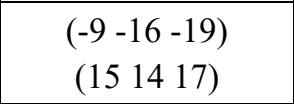 & 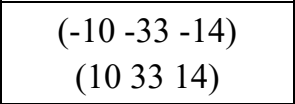 & 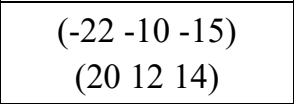 & $\begin{array}{l}\left(\begin{array}{lll}-9 & -1 & -22\end{array}\right) \\
\left(\begin{array}{llll}9 & 13 & 22\end{array}\right)\end{array}$ & 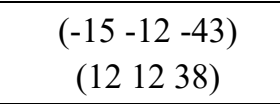 \\
\hline $\begin{array}{c}\text { Reflections } \\
\text { collected / unique }\end{array}$ & 17074 / 4869 & $72433 / 4903$ & $11879 / 4443$ & $54222 / 6041$ & $34080 / 4471$ \\
\hline $\begin{array}{l}\text { Reflections collected } \\
(I>2 \sigma(I))\end{array}$ & 2605 & 2819 & 2172 & 2928 & 2626 \\
\hline Restraints / parameters & $0 / 239$ & $1 / 245$ & $0 / 217$ & $0 / 301$ & $0 / 191$ \\
\hline Goodness of fit $\left(\mathrm{F}^{2}\right)$ & 1.024 & 1.030 & 0.994 & 0.971 & 1.017 \\
\hline $\mathrm{R}_{1}, \mathrm{wR}_{2}$ (all data) & $0.1164 / 0.1861$ & $0.1179 / 0.1824$ & $0.1293 / 0.1361$ & $0.1609 / 0.2348$ & $0.1051 / 0.1742$ \\
\hline $\left.\mathrm{R}_{1}, \mathrm{wR}_{2}(\mathrm{I}>2 \sigma(\mathrm{I})]\right)$ & $0.0565 / 0.1499$ & $0.0588 / 0.1493$ & $0.0550 / 0.1080$ & $0.0749 / 0.1821$ & $0.0568 / 0.1426$ \\
\hline $\begin{array}{c}\text { Largest diff. peak and } \\
\text { hole/e. } \AA^{-3}\end{array}$ & $0.197 /-0.183$ & $0.209 /-0.302$ & $0.194 /-0.235$ & $0.218 /-0.373$ & $0.235 /-0.378$ \\
\hline
\end{tabular}

\section{$X$-ray Crystal Structure Determination}

Crystallization of the compounds was carried out by cooling a solution of the compound in pentane (10 $\mathrm{mg} / \mathrm{mL}$ ) at $-20^{\circ} \mathrm{C}$ during several days. Suitable Crystals were selected under polarizing optical microscope and mounted on MicroMount needles (MiTiGen) for single-crystal X-ray diffraction experiments. X-ray intensity data were collected on a Bruker APEX II Quazar diffractometer (4 circle Kappa goniometer, CCD detector) using I $\mu$ s microfocus source (Mo- $K_{\alpha}$ radiation with $\lambda=0.71073 \AA$ ) at $296 \mathrm{~K}$. The structure solutions were obtained by direct methods, developed by successive difference Fourier syntheses, and refined by fullmatrix least-squares on all $\mathrm{F}^{2}$ data using SHELX program suite ${ }^{[1]}$ in Bruker APEX2 interface. Details of the structure determinations are given in Table 1.

Crystallographic data (excluding structure factors) for all structures have been deposited at the Cambridge Crystallographic Data Centre, CCDC 2008880 for $\mathrm{C}_{19} \mathrm{H}_{30} \mathrm{~N}_{2} \mathrm{O}_{2} \mathrm{Si}$ (3a), 2008881 for $\mathrm{C}_{20} \mathrm{H}_{32} \mathrm{~N}_{2} \mathrm{O}_{3} \mathrm{Si}$ (3b), 2008882 for $\mathrm{C}_{17} \mathrm{H}_{28} \mathrm{~N}_{2} \mathrm{O}_{3} \mathrm{Si}$ (3e), 2008883 for $\mathrm{C}_{26} \mathrm{H}_{36} \mathrm{~N}_{2} \mathrm{O}_{3} \mathrm{Si}$ (4a) and 2008884 for $\mathrm{C}_{16} \mathrm{H}_{34} \mathrm{~N}_{2} \mathrm{OSi}$ (10). Copies of the data can be obtained, free of charge, on application to CCDC, 12 Union Road, Cambridge CB2 1EZ, UK, (fax: +44 1223336033 or e-mail: deposit@,ccdc.cam.ac.uk).

[1] G.M. Sheldrick, " SHELXL for crystal structure determination, Göttingen Univ., Germany (2014). 


\section{Computational data}

\section{DFT study}

The full geometry optimization was performed with the hybrid meta exchange correlation DFT functional M06-2X $\mathrm{X}^{[2]}$ with the $6-311+\mathrm{G}(\mathrm{d}, \mathrm{p})$ basis set ${ }^{[3,4]}$ using the Gaussian $09^{\circledR}$ suite of programs. ${ }^{[5]}$ M06-2X was described to provide reasonable energetics in the case of systems involving non-bonded interactions. ${ }^{[2,6]}$

Solvent effects were included using the SMD solvent $\operatorname{model}^{7}$ for the description of THF as the solvent. Frequency calculations were used to characterize stationary points by the number of negative eigenvalues of their analytic Hessian matrix. All reported data in table S1 include electronic energy (E), zero-point energies (ZPEs), enthalpy and Gibbs free energy at $248 \mathrm{~K}\left(-25^{\circ} \mathrm{C}\right)$.

\begin{tabular}{|c|c|c|c|c|}
\hline & E & ZPE & $\mathrm{H}^{\circ}{ }_{248}$ & $\mathbf{G}^{\circ}{ }_{248}$ \\
\hline THF & -232.413090967 & -232.295408 & -232.290909 & -232.31878 \\
\hline LDA & -299.2967647 & -299.103444 & -299.094789 & -299.131249 \\
\hline LDA-3THF & -996.597419755 & -996.043488 & -996.022234 & -996.088292 \\
\hline Diisopropylamine & -292.358058123 & -292.15195 & -292.144386 & -292.1785 \\
\hline 1 & -945.819499235 & -945.47294 & -945.456524 & -945.510114 \\
\hline 6 & -952.771494712 & -952.434809 & -952.417889 & -952.47259 \\
\hline 7 & -1245.15718914 & -1244.610696 & -1244.58707 & -1244.656361 \\
\hline $7^{\prime}$ & -1245.16122844 & -1244.614193 & -1244.59076 & -1244.659001 \\
\hline 8 & -1252.11635767 & -1251.580263 & -1251.556403 & -1251.6255 \\
\hline 6-2THF & -1417.66714443 & -1417.090964 & -1417.065214 & -1417.140671 \\
\hline 7-2THF & -1710.03921707 & -1709.253061 & -1709.220851 & -1709.309647 \\
\hline 7'-2THF & -1710.04214157 & -1709.255809 & -1709.223441 & -1709.313229 \\
\hline 8-4THF & -2181.86983198 & -2180.853198 & -2180.811913 & -2180.919875 \\
\hline 14 & -1238.15520831 & -1237.598054 & -1237.575292 & -1237.643229 \\
\hline $14^{\prime}$ & -1238.17051931 & -1237.612746 & -1237.589838 & -1237.657209 \\
\hline
\end{tabular}

Table S1. Electronic energy (E), zero-point energy (ZPE), enthalpy at 248K $\left(\mathrm{H}^{\circ}{ }_{248}\right)$ and Gibbs free energy at $248 \mathrm{~K}\left(\mathrm{G}^{\circ} 248\right)$, in Hartree.

[2] Y. Zhao, D. G. Truhlar, Theor. Chem. Acc. 2008, 120, 215.

${ }^{[3]}$ A. D. McLean and G. S. Chandler, J. Chem. Phys. 1980, 5639.

${ }^{[4]}$ K. Raghavachari, J. S. Binkley, R. Seeger, and J. A. Pople, J. Chem. Phys. 1980, 650.

${ }^{[5]}$ M. J. Frisch, G. W. T., H. B. Schlegel, G. E. Scuseria, M. A. Robb, J. R. Cheeseman, J. A. Montgomery Jr., T. Vreven, K. N. Kudin, J. C. Burant, J. M. Millam, S. S. Iyengar, J. Tomasi, V. Barone, B., Mennucci, M. Cossi, G. Scalmani, N. Rega, G. A. Petersson, H. Nakatsuji, M. Hada, M. Ehara, K. Toyota, R. Fukuda, J. Hasegawa, M. Ishida, T. Nakajima, Y. Honda, O. Kitao, H. Nakai, M. Klene, X. Li, J. E. Knox, H. P. Hratchian, J. B. Cross, V. Bakken, C. Adamo, J. Jaramillo, R. Gomperts, R. E. Stratmann, O. Yazyev, A. J. Austin, R. Cammi, C. Pomelli, J. W. Ochterski, P. Y. Ayala, K. Morokuma, G. A. Voth, P. Salvador, J. J. Dannenberg, G. Zakrzewski, S. Dapprich, A. D. Daniels, M. C. Strain, O. Farkas, D. K. Malick, A. D. Rabuck, K. Raghavachari, J. B. Foresman, J. V. Ortiz, Q. Cui, A. G. Baboul, S. Clifford, J. Cioslowski, B. B. Stefanov, G. Liu, A. Liashenko, P. Piskorz, I. Komaromi, R. L. Martin, D. J. Fox, T. Keith, M. A. Al-Laham, C. Y. Peng, A. Nanayakkara, M. Challacombe, P. M. W. Gill, B. Johnson, W. Chen, M. W. Wong, C. Gonzalez, J. A. Pople, Gaussian 09, Gaussian, Inc.: Pittsburgh, PA, 2003.

[6] Y.-Z. Tan, Z.-J. Liao, Z.-Z. Qian, R.-T. Chen, X. Wu, H. Liang, X., Han, F. Zhu, S.-J. Zhou, Z. Zheng, X. Lu, S.-Y. Xie, R.-B. Huang, L.-S. Zheng, Nat. Mater. 2008, 7, 790.

${ }^{[7]}$ A. V. Marenich, C. J. Cramer, and D. G. Truhlar, J. Phys. Chem. B, 113 (2009) 6378-96. 


\section{XYZ data of the DFT calculated conformers}

\section{a. THF}

$\mathrm{E}=-232.413090967 \mathrm{au}$

Charge: 0, spin: singlet. 5 lowest frequencies: 20.3917, 297.6401, 647,9389, 650.4635, 805.8899.

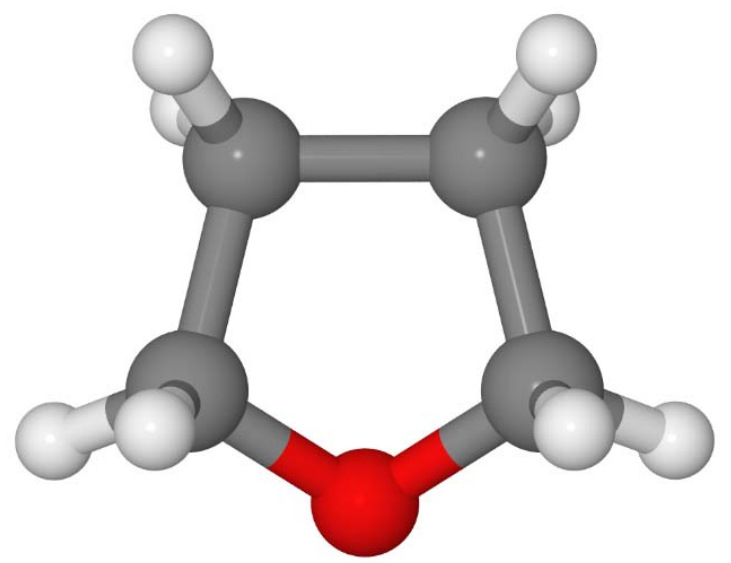

$\begin{array}{lrrr}\mathrm{O} & 0.00015 & -1.19612 & -0.30412 \\ \mathrm{C} & -1.12702 & -0.46795 & 0.16179 \\ \mathrm{C} & -0.77423 & 1.01109 & -0.05172 \\ \mathrm{C} & 0.77384 & 1.01142 & -0.05129 \\ \mathrm{C} & 1.12725 & -0.46762 & 0.16147 \\ \mathrm{H} & -1.28553 & -0.67955 & 1.22809 \\ \mathrm{H} & -2.00235 & -0.80007 & -0.39657 \\ \mathrm{H} & -1.15992 & 1.36248 & -1.00955 \\ \mathrm{H} & -1.19519 & 1.63975 & 0.73339 \\ \mathrm{H} & 1.19404 & 1.63971 & 0.73454 \\ \mathrm{H} & 1.15993 & 1.36376 & -1.00860 \\ \mathrm{H} & 1.28638 & -0.67955 & 1.22760 \\ \mathrm{H} & 2.00242 & -0.79922 & -0.39745\end{array}$

b. LDA

$\mathrm{E}=-299.296764693 \mathrm{au}$

Charge: 0, spin: singlet. 5 lowest frequencies: 49.9692, 85.4168, 96.3439, 141.8813, 192.9190.

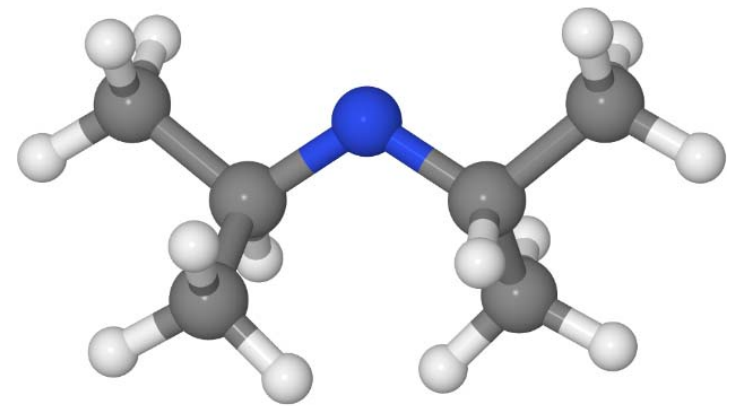

$\begin{array}{lrrr}\mathrm{N} & -0.00001 & 0.58736 & 0.00029 \\ \mathrm{C} & 1.19602 & -0.19448 & 0.24647 \\ \mathrm{H} & 1.10686 & -0.80335 & 1.16714 \\ \mathrm{C} & 1.55434 & -1.17279 & -0.88425 \\ \mathrm{H} & 2.49671 & -1.68988 & -0.67533 \\ \mathrm{H} & 1.65987 & -0.62730 & -1.82938 \\ \mathrm{H} & 0.78305 & -1.93567 & -1.02062 \\ \mathrm{C} & 2.36696 & 0.76231 & 0.47079 \\ \mathrm{H} & 3.29269 & 0.22865 & 0.70412 \\ \mathrm{H} & 2.15610 & 1.44317 & 1.30302\end{array}$




$\begin{array}{lrrr}\mathrm{H} & 2.54231 & 1.36029 & -0.43210 \\ \mathrm{C} & -1.19596 & -0.19445 & -0.24650 \\ \mathrm{H} & -1.10715 & -0.80193 & -1.16814 \\ \mathrm{C} & -2.36715 & 0.76251 & -0.46887 \\ \mathrm{H} & -2.15672 & 1.44474 & -1.30015 \\ \mathrm{H} & -2.54230 & 1.35900 & 0.43504 \\ \mathrm{H} & -3.29288 & 0.22916 & -0.70289 \\ \mathrm{C} & -1.55368 & -1.17449 & 0.88289 \\ \mathrm{H} & -0.78249 & -1.93776 & 1.01753 \\ \mathrm{H} & -2.49629 & -1.69104 & 0.67372 \\ \mathrm{H} & -1.65850 & -0.63051 & 1.82897 \\ \mathrm{Li} & -0.00142 & 2.40307 & -0.00204\end{array}$

\section{c. LDA-3THF}

$\mathrm{E}=-996.597419755 \mathrm{au}$

Charge: 0, spin: singlet. 5 lowest frequencies: 28.9053, 37.2422, 50.9805, 60.8400, 62.2670.

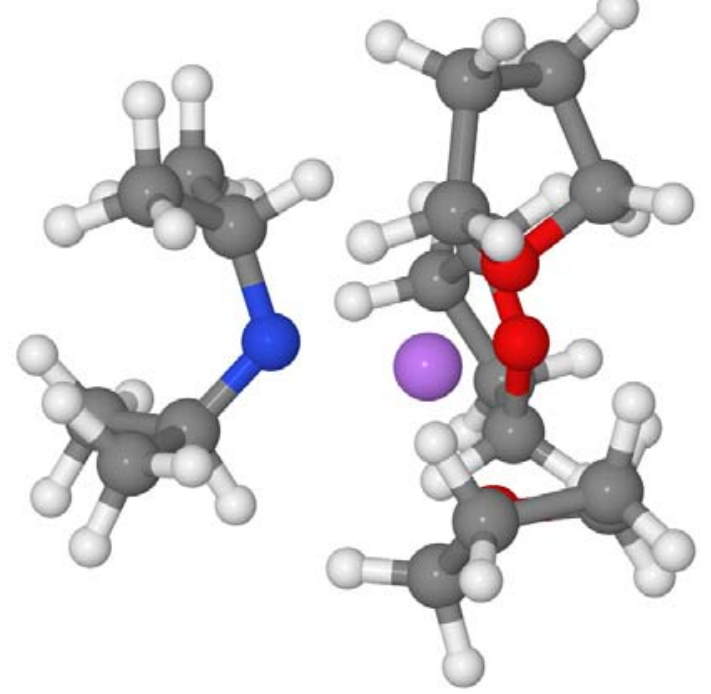
$\mathrm{N} \quad-1.47285-0.56559 \quad-0.27913$
C $\quad-2.18393 \quad-1.08520 \quad-1.43061$
$\mathrm{H} \quad-1.63060 \quad-0.69111 \quad-2.30240$
C $\quad-2.16164-2.62556-1.62033$
H $-2.42635 \quad-2.89449-2.65339$
H $\quad-2.87021-3.13708-0.95936$
H $\quad-1.16132-3.02455-1.41056$
C $\quad-3.63966-0.61149-1.63190$
H $\quad-4.31640 \quad-1.06769-0.89899$
H $\quad-4.00547-0.88978-2.62992$
$\mathrm{H} \quad-3.71244 \quad 0.47827 \quad-1.53213$
C $\quad-2.05918 \quad-0.68235 \quad 1.04184$
$\mathrm{H} \quad-1.23711 \quad-0.41962 \quad 1.73530$
$\begin{array}{llll}\text { C } & -2.53620 & -2.08244 & 1.48973\end{array}$
$\begin{array}{llll}\mathrm{H} & -1.78299 & -2.84994 & 1.27538\end{array}$
H $\quad-3.46411 \quad-2.37096 \quad 0.98041$
H $\quad-2.74209 \quad-2.09560 \quad 2.56932$
$\begin{array}{llll}\text { C } & -3.18606 & 0.32400 & 1.39426\end{array}$
$\begin{array}{llll}\mathrm{H} & -4.14467 & 0.05246 & 0.93843\end{array}$
$\mathrm{H} \quad-2.92286 \quad 1.32934 \quad 1.04605$

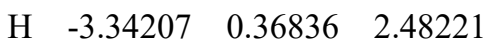
$\begin{array}{llll}\mathrm{Li} & 0.27783 & 0.15057 & -0.35435\end{array}$
$\begin{array}{llll}\text { O } & 1.42709 & -0.38417 & 1.14419\end{array}$
O $\quad 0.63168 \quad 2.09165 \quad-0.23870$
$\begin{array}{llll}\text { O } & 1.45786 & -0.12265 & -1.91101\end{array}$
$\begin{array}{llll}\text { C } & 2.13146 & 0.46498 & 2.04689\end{array}$
$\mathrm{H} \quad 2.09874 \quad 1.48134 \quad 1.65045$
H $3.18009 \quad 0.13407 \quad 2.11150$
$\begin{array}{llll}\text { C } & 1.21341 & -1.61188 & 1.84629\end{array}$ 


$\begin{array}{rrrr}\text { H } & 2.10587 & -2.24312 & 1.72755 \\ \mathrm{H} & 0.35772 & -2.10618 & 1.38179 \\ \mathrm{C} & 1.40779 & 0.26604 & 3.37640 \\ \mathrm{H} & 0.51948 & 0.90563 & 3.40941 \\ \mathrm{H} & 2.03940 & 0.50832 & 4.23490 \\ \mathrm{C} & 1.00314 & -1.22488 & 3.32474 \\ \mathrm{H} & -0.03643 & -1.36846 & 3.63161 \\ \mathrm{H} & 1.63392 & -1.83648 & 3.97552 \\ \mathrm{C} & -0.20070 & 2.66270 & 0.79055 \\ \mathrm{H} & 0.44165 & 3.22419 & 1.48144 \\ \mathrm{H} & -0.68667 & 1.84428 & 1.33344 \\ \mathrm{C} & 0.36628 & 2.74075 & -1.49701 \\ \mathrm{H} & 1.32129 & 2.88406 & -2.00988 \\ \mathrm{H} & -0.27253 & 2.08700 & -2.10513 \\ \mathrm{C} & -1.18110 & 3.58172 & 0.06960 \\ \mathrm{H} & -1.52132 & 4.40428 & 0.70401 \\ \mathrm{H} & -2.05288 & 3.01122 & -0.27004 \\ \mathrm{C} & -0.35371 & 4.03277 & -1.13681 \\ \mathrm{H} & -0.96123 & 4.41237 & -1.96223 \\ \mathrm{H} & 0.36651 & 4.80534 & -0.84367 \\ \mathrm{C} & 2.82416 & 0.12246 & -1.55638 \\ \mathrm{H} & 3.34337 & 0.53419 & -2.43230 \\ \mathrm{H} & 2.82551 & 0.86606 & -0.75622 \\ \mathrm{C} & 1.43361 & -1.42720 & -2.49248 \\ \mathrm{H} & 1.83962 & -1.37363 & -3.51355 \\ \mathrm{H} & 0.39371 & -1.75668 & -2.53137 \\ \mathrm{C} & 3.41295 & -1.24330 & -1.14753 \\ \mathrm{H} & 3.59415 & -1.29034 & -0.07052 \\ \mathrm{H} & 4.36063 & -1.42348 & -1.66257 \\ \mathrm{C} & 2.32955 & -2.25682 & -1.57835 \\ \mathrm{H} & 2.74104 & -3.13468 & -2.08326 \\ \mathrm{H} & 1.74942 & -2.59404 & -0.71338\end{array}$

\section{d. Diisopropylamine}

$\mathrm{E}=-292.358058123 \mathrm{au}$

Charge: 0, spin: singlet. 5 lowest frequencies: 36.1415, 107.5924, 212.4912, 225.4529, 231.4986.

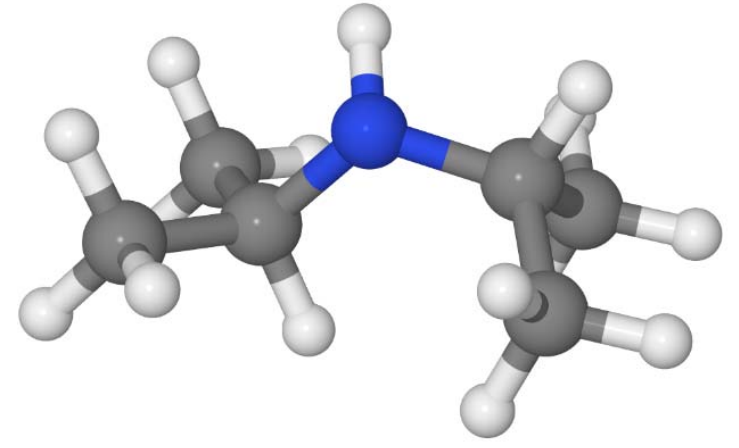
$\begin{array}{llll}\mathrm{H} & -0.22171 & 0.48368 & 1.58880\end{array}$
$\begin{array}{llll}\mathrm{N} & -0.02979 & -0.17820 & 0.83894\end{array}$
C $\quad 1.38996-0.05459 \quad 0.44681$
H $\quad 1.94159-0.08749 \quad 1.39630$
C $\quad 1.83692-1.26993-0.37779$
H $\quad 2.91765-1.23136-0.55968$
$\mathrm{H} \quad 1.34141-1.30296-1.35614$
$\begin{array}{llll}\mathrm{H} & 1.60705 & -2.20073 & 0.15229\end{array}$
$\begin{array}{llll}\text { C } & 1.76739 & 1.26606 & -0.25512\end{array}$
$\begin{array}{llll}\mathrm{H} & 2.85044 & 1.31312 & -0.42717\end{array}$
$\begin{array}{llll}\mathrm{H} & 1.48640 & 2.13154 & 0.35740\end{array}$
$\begin{array}{llll}\mathrm{H} & 1.27366 & 1.36364 & -1.23019\end{array}$
$\begin{array}{llll}\text { C } & -1.06423 & -0.04121 & -0.21191\end{array}$
$\begin{array}{llll}\mathrm{H} & -0.56940 & -0.14281 & -1.18565\end{array}$
$\begin{array}{llll}\text { C } & -2.08954 & -1.17935 & -0.08998\end{array}$
$\begin{array}{llll}\text { H } & -2.60596 & -1.12837 & 0.87754\end{array}$ 


$\begin{array}{rrrr}\mathrm{H} & -1.59256 & -2.15346 & -0.15495 \\ \mathrm{H} & -2.84541 & -1.11997 & -0.88414 \\ \mathrm{C} & -1.76285 & 1.32830 & -0.17366 \\ \mathrm{H} & -1.04831 & 2.14709 & -0.31186 \\ \mathrm{H} & -2.26591 & 1.47483 & 0.79255 \\ \mathrm{H} & -2.52632 & 1.40504 & -0.95783\end{array}$

\section{e. 1}

$\mathrm{E}=-945.819499235 \mathrm{au}$

Charge: 0, spin: singlet. 5 lowest frequencies: $36.2768,59.3271,70.0543,87.3327,100.5564$.

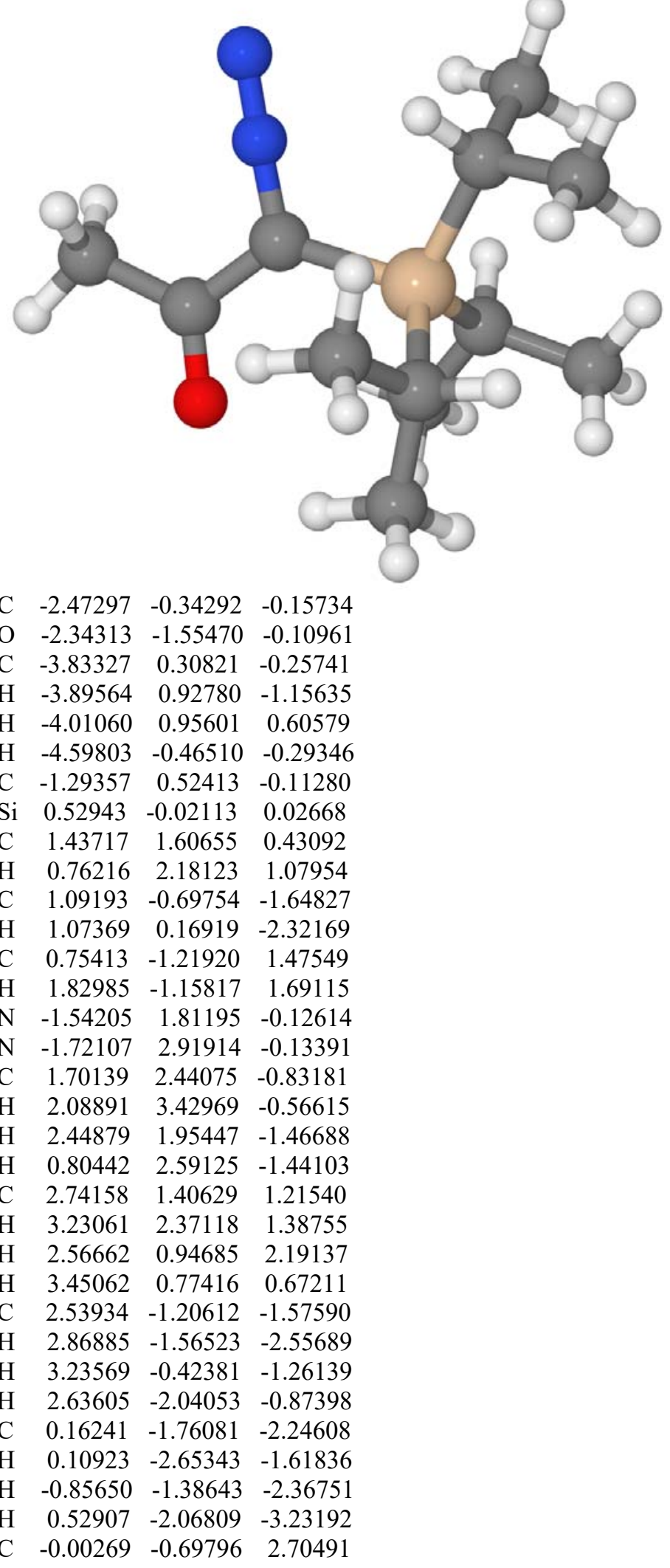




$\begin{array}{crcc}\mathrm{H} & -1.08339 & -0.78597 & 2.55386 \\ \mathrm{H} & 0.25608 & -1.28060 & 3.59525 \\ \mathrm{H} & 0.21912 & 0.35278 & 2.91896 \\ \mathrm{C} & 0.42236 & -2.69374 & 1.21881 \\ \mathrm{H} & -0.62515 & -2.81896 & 0.93952 \\ \mathrm{H} & 1.03859 & -3.11834 & 0.42275 \\ \mathrm{H} & 0.60899 & -3.28000 & 2.12588\end{array}$

\section{f. 6}

$\mathrm{E}=-952.771494712 \mathrm{au}$

Charge: 0, spin: singlet. 5 lowest frequencies: $35.9720,51.7144,70.4257,80.9056,89.8152$.

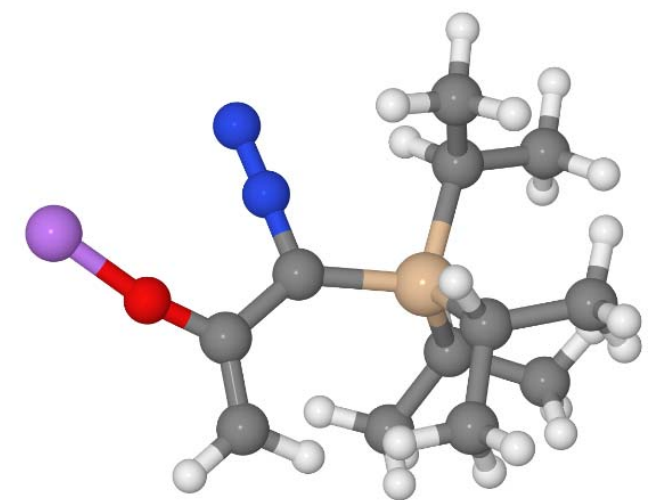

$\begin{array}{lrrr}\mathrm{C} & -2.27806 & -2.10888 & 0.29697 \\ \mathrm{H} & -3.08620 & -2.78926 & 0.53820 \\ \mathrm{H} & -1.28571 & -2.50810 & 0.15727 \\ \mathrm{C} & -2.53704 & -0.78511 & 0.16133 \\ \mathrm{O} & -3.71542 & -0.24692 & 0.27290 \\ \mathrm{C} & -1.41305 & 0.17596 & -0.14375 \\ \mathrm{~N} & -1.86827 & 1.35531 & -0.43627 \\ \mathrm{~N} & -2.28614 & 2.37882 & -0.68015 \\ \mathrm{Si} & 0.49265 & 0.05754 & -0.08087 \\ \mathrm{C} & 1.01550 & 1.87245 & -0.35793 \\ \mathrm{H} & 0.37077 & 2.21678 & -1.17780 \\ \mathrm{C} & 1.16562 & -0.95817 & -1.54499 \\ \mathrm{H} & 1.14146 & -0.23128 & -2.36996 \\ \mathrm{C} & 2.62606 & -1.39176 & -1.34433 \\ \mathrm{H} & 3.28249 & -0.56749 & -1.05675 \\ \mathrm{H} & 3.02178 & -1.82428 & -2.26999 \\ \mathrm{H} & 2.69842 & -2.16060 & -0.56913 \\ \mathrm{C} & 0.72685 & 2.75625 & 0.86525 \\ \mathrm{H} & 0.86133 & 3.81463 & 0.61714 \\ \mathrm{H} & 1.41048 & 2.52584 & 1.68747 \\ \mathrm{H} & -0.29174 & 2.63488 & 1.24606 \\ \mathrm{C} & 2.45559 & 2.07983 & -0.84877 \\ \mathrm{H} & 2.64570 & 1.55232 & -1.78690 \\ \mathrm{H} & 3.19739 & 1.74442 & -0.11978 \\ \mathrm{H} & 2.63717 & 3.14515 & -1.02976 \\ \mathrm{C} & 0.33193 & -2.16604 & -1.98562 \\ \mathrm{H} & 0.73590 & -2.57888 & -2.91689 \\ \mathrm{H} & -0.71546 & -1.90797 & -2.15587 \\ \mathrm{H} & 0.36382 & -2.96413 & -1.23780 \\ \mathrm{C} & 1.05298 & -0.53009 & 1.64512 \\ \mathrm{H} & 0.45135 & 0.07319 & 2.33847 \\ \mathrm{C} & 2.53229 & -0.21323 & 1.92709 \\ \mathrm{H} & 2.78090 & 0.83760 & 1.77279 \\ \mathrm{H} & 3.19768 & -0.81215 & 1.30018 \\ \mathrm{H} & 2.76629 & -0.45503 & 2.96967 \\ \mathrm{C} & 0.79070 & -2.00988 & 1.96168 \\ \mathrm{H} & 1.22560 & -2.66834 & 1.20206 \\ \mathrm{H} & -0.27321 & -2.23256 & 2.03363\end{array}$




$\begin{array}{lrrr}\mathrm{H} & 1.25193 & -2.27259 & 2.92055 \\ \mathrm{Li} & -5.09905 & 0.71027 & 0.29289\end{array}$

g. 7

$E=-1245.15718914$ au

Charge: 0, spin: singlet. 5 lowest frequencies: 22.4318, 25.0586, 48.5887, 53.0364, 59.0798.

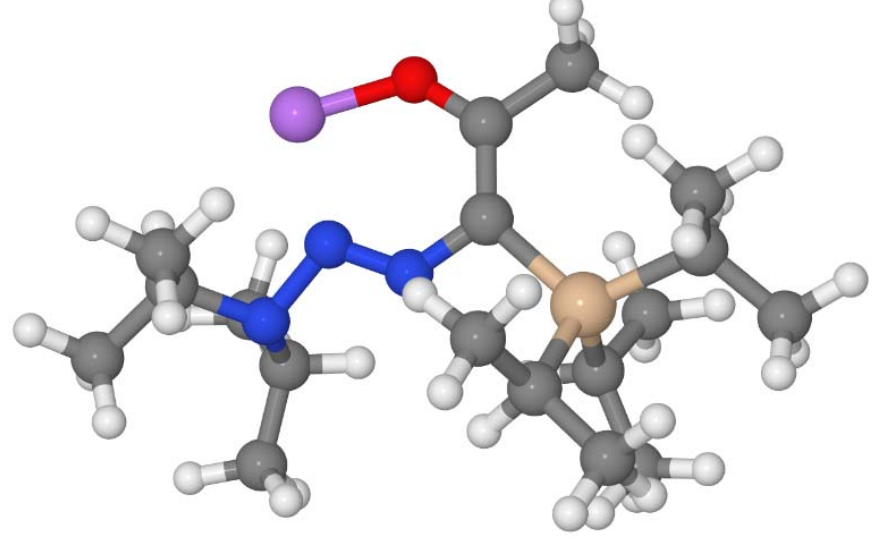

\begin{tabular}{|c|c|c|c|}
\hline $\mathrm{C}$ & 0.44494 & 1.02998 & -0.34933 \\
\hline $\mathrm{C}$ & 0.39923 & 2.34409 & -0.86400 \\
\hline$\Omega$ & -0.66840 & 3.01341 & -1.0203 \\
\hline$N$ & -0.69783 & 0.31669 & -0.028 \\
\hline $\mathrm{N}$ & -1.86238 & 0.77048 & -0.087 \\
\hline $\mathrm{N}$ & -2.84273 & -0.21029 & 0.292 \\
\hline $\mathrm{C}$ & -2.80673 & -1.35768 & -0.657 \\
\hline $\mathrm{H}$ & -1.73833 & -1.51271 & -0.809 \\
\hline $\mathrm{C}$ & -4.11341 & 0.52587 & 0.4406 \\
\hline $\mathrm{H}$ & -4.33460 & 1.08087 & -0.4896 \\
\hline $\mathrm{Li}$ & -2.32032 & 2.56426 & -0.6206 \\
\hline $\mathrm{C}$ & 1.64843 & 3.06763 & -1.3059 \\
\hline $\mathrm{H}$ & 2.54986 & 2.46682 & -1.2813 \\
\hline $\mathrm{H}$ & 1.78819 & 3.94887 & -0.6729 \\
\hline $\mathrm{H}$ & 1.48709 & 3.42634 & -2.325 \\
\hline $\mathrm{Si}$ & 1.85505 & -0.18679 & 0.0271 \\
\hline $\mathrm{C}$ & 3.60934 & 0.56296 & -0.0102 \\
\hline $\mathrm{H}$ & 3.71352 & 1.03704 & -0.9921 \\
\hline $\mathrm{C}$ & 3.79788 & 1.63543 & 1.0724 \\
\hline $\mathrm{H}$ & 3.82506 & 1.17505 & 2.0650 \\
\hline $\mathrm{H}$ & 2.99243 & 2.37570 & 1.0775 \\
\hline $\mathrm{H}$ & 4.74468 & 2.17030 & 0.9353 \\
\hline $\mathrm{C}$ & 4.75003 & -0.46488 & 0.0661 \\
\hline $\mathrm{H}$ & 4.69478 & -1.20211 & -0.7377 \\
\hline $\mathrm{H}$ & 4.75950 & -1.00406 & $1.014 \mathrm{C}$ \\
\hline $\mathrm{H}$ & 5.71564 & 0.04613 & -0.025 \\
\hline $\mathrm{C}$ & 1.43932 & -0.93700 & 1.7386 \\
\hline $\mathrm{H}$ & 0.62750 & -1.64490 & 1.5230 \\
\hline $\mathrm{C}$ & 0.89640 & 0.08790 & 2.7452 \\
\hline $\mathrm{H}$ & 0.66182 & -0.40100 & 3.6981 \\
\hline $\mathrm{H}$ & -0.01455 & 0.57290 & 2.3884 \\
\hline $\mathrm{H}$ & 1.63299 & 0.87048 & 2.9542 \\
\hline $\mathrm{C}$ & 2.58530 & -1.72339 & 2.3921 \\
\hline $\mathrm{H}$ & 3.04973 & -2.44547 & 1.7163 \\
\hline $\mathrm{H}$ & 2.21774 & -2.27477 & 3.2652 \\
\hline $\mathrm{H}$ & 3.36983 & -1.04767 & 2.7465 \\
\hline $\mathrm{C}$ & 1.70299 & -1.54333 & -1.305 \\
\hline $\mathrm{H}$ & 0.61693 & -1.63993 & -1.438 \\
\hline $\mathrm{C}$ & 2.29084 & -1.06255 & -2.638 \\
\hline $\mathrm{H}$ & 2.05876 & -1.76300 & -3.448 \\
\hline $\mathrm{H}$ & 3.38105 & -0.97730 & -2.584 \\
\hline $\mathrm{H}$ & 1.89595 & -0.08202 & -2.926 \\
\hline $\mathrm{C}$ & 2.23769 & -2.93145 & -0.934 \\
\hline
\end{tabular}




$\begin{array}{lrrr}\mathrm{H} & 1.75089 & -3.32599 & -0.03799 \\ \mathrm{H} & 3.31583 & -2.92124 & -0.74940 \\ \mathrm{H} & 2.05545 & -3.64381 & -1.74762 \\ \mathrm{C} & -5.29791 & -0.38967 & 0.73307 \\ \mathrm{H} & -6.15676 & 0.22896 & 1.00331 \\ \mathrm{H} & -5.07186 & -1.04404 & 1.57910 \\ \mathrm{H} & -5.58951 & -1.00225 & -0.11804 \\ \mathrm{C} & -3.98735 & 1.51411 & 1.60430 \\ \mathrm{H} & -4.88638 & 2.13097 & 1.67093 \\ \mathrm{H} & -3.12172 & 2.17659 & 1.53052 \\ \mathrm{H} & -3.87811 & 0.95759 & 2.53953 \\ \mathrm{C} & -3.32368 & -2.66127 & -0.05121 \\ \mathrm{H} & -4.41067 & -2.73006 & -0.02467 \\ \mathrm{H} & -2.94061 & -2.78484 & 0.96422 \\ \mathrm{H} & -2.95821 & -3.49582 & -0.65615 \\ \mathrm{C} & -3.42673 & -1.04857 & -2.02151 \\ \mathrm{H} & -4.50873 & -0.90803 & -1.96794 \\ \mathrm{H} & -3.23409 & -1.87822 & -2.70669 \\ \mathrm{H} & -2.98235 & -0.14750 & -2.45461\end{array}$

h. 7

$\mathrm{E}=-1245.16122844 \mathrm{au}$

Charge: 0, spin: singlet. 5 lowest frequencies: 23.0020, 39.6524, 54.1765, 56.6625, 70.7481 .

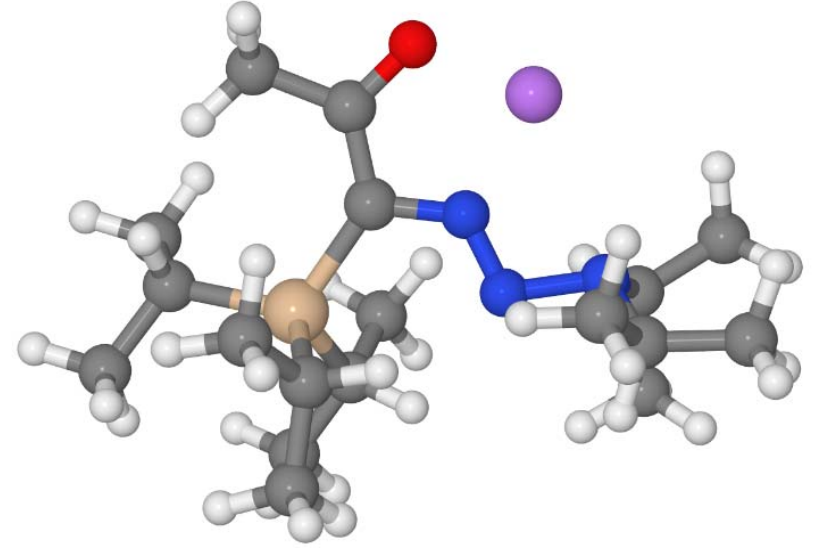

$\begin{array}{lrrr}\mathrm{C} & -0.57874 & 1.29831 & 0.28005 \\ \mathrm{C} & -0.86777 & 2.67833 & 0.47977 \\ \mathrm{O} & 0.04105 & 3.56016 & 0.60761 \\ \mathrm{~N} & 0.79975 & 1.13525 & 0.29635 \\ \mathrm{~N} & 1.33745 & 0.01307 & 0.23371 \\ \mathrm{~N} & 2.77821 & 0.18424 & 0.20971 \\ \mathrm{C} & 3.17194 & 0.30005 & -1.22717 \\ \mathrm{H} & 2.41662 & 0.97623 & -1.63661 \\ \mathrm{C} & 3.32754 & -0.96380 & 0.96732 \\ \mathrm{H} & 2.92001 & -1.89808 & 0.55388 \\ \mathrm{Li} & 1.72595 & 2.84388 & 0.61809 \\ \mathrm{C} & -2.27050 & 3.21945 & 0.56992 \\ \mathrm{H} & -3.04454 & 2.46300 & 0.61614 \\ \mathrm{H} & -2.45246 & 3.86523 & -0.29440 \\ \mathrm{H} & -2.33054 & 3.85041 & 1.45987 \\ \mathrm{Si} & -1.64100 & -0.26258 & 0.03503 \\ \mathrm{C} & -3.49483 & 0.12664 & -0.20651 \\ \mathrm{H} & -3.78092 & 0.74834 & 0.64857 \\ \mathrm{C} & -3.76213 & 0.91256 & -1.49931 \\ \mathrm{H} & -3.63604 & 0.26455 & -2.37192 \\ \mathrm{H} & -3.08827 & 1.76417 & -1.62663 \\ \mathrm{H} & -4.79023 & 1.29190 & -1.52220 \\ \mathrm{C} & -4.42459 & -1.09708 & -0.14428 \\ \mathrm{H} & -4.32602 & -1.64578 & 0.79452 \\ \mathrm{H} & -4.24433 & -1.79718 & -0.96161\end{array}$




$\begin{array}{lrrr}\mathrm{H} & -5.46826 & -0.77047 & -0.22264 \\ \mathrm{C} & -0.93591 & -1.20842 & -1.47382 \\ \mathrm{H} & -0.05531 & -1.72351 & -1.07464 \\ \mathrm{C} & -0.46139 & -0.27782 & -2.59788 \\ \mathrm{H} & -0.00605 & -0.85562 & -3.41132 \\ \mathrm{H} & 0.27664 & 0.44794 & -2.24881 \\ \mathrm{H} & -1.29846 & 0.28277 & -3.02671 \\ \mathrm{C} & -1.86957 & -2.27693 & -2.06120 \\ \mathrm{H} & -2.26004 & -2.96190 & -1.30471 \\ \mathrm{H} & -1.33196 & -2.87719 & -2.80456 \\ \mathrm{H} & -2.72278 & -1.82220 & -2.57259 \\ \mathrm{C} & -1.41047 & -1.29588 & 1.62129 \\ \mathrm{H} & -0.35371 & -1.14701 & 1.87159 \\ \mathrm{C} & -2.24185 & -0.72703 & 2.77867 \\ \mathrm{H} & -1.98070 & -1.21399 & 3.72510 \\ \mathrm{H} & -3.31353 & -0.88185 & 2.61896 \\ \mathrm{H} & -2.07772 & 0.34848 & 2.90547 \\ \mathrm{C} & -1.62795 & -2.80615 & 1.46960 \\ \mathrm{H} & -0.96659 & -3.23463 & 0.71109 \\ \mathrm{H} & -2.65713 & -3.04771 & 1.18815 \\ \mathrm{H} & -1.42089 & -3.32153 & 2.41497 \\ \mathrm{C} & 4.85087 & -1.02020 & 0.92065 \\ \mathrm{H} & 5.19527 & -1.75655 & 1.65062 \\ \mathrm{H} & 5.28154 & -0.05131 & 1.18956 \\ \mathrm{H} & 5.23875 & -1.31921 & -0.05200 \\ \mathrm{C} & 2.88061 & -0.83645 & 2.42253 \\ \mathrm{H} & 3.23416 & -1.69497 & 2.99853 \\ \mathrm{H} & 1.79484 & -0.78912 & 2.51278 \\ \mathrm{H} & 3.30312 & 0.07263 & 2.86279 \\ \mathrm{C} & 4.51022 & 1.01151 & -1.42622 \\ \mathrm{H} & 5.37206 & 0.36997 & -1.24713 \\ \mathrm{H} & 4.58595 & 1.88347 & -0.77181 \\ \mathrm{H} & 4.57026 & 1.35818 & -2.46160 \\ \mathrm{C} & 3.08584 & -1.00954 & -2.01214 \\ \mathrm{H} & 3.86524 & -1.71561 & -1.71501 \\ \mathrm{H} & 3.21079 & -0.80532 & -3.07896 \\ \mathrm{H} & 2.11311 & -1.48670 & -1.86846 \\ & & & \\ & & & \end{array}$

i. 8

$\mathrm{E}=-1252.11635767 \mathrm{au}$

Charge: 0 , spin: singlet. 5 lowest frequencies: $21.4281,38.6593,49.5938,60.5420,64.8329$.

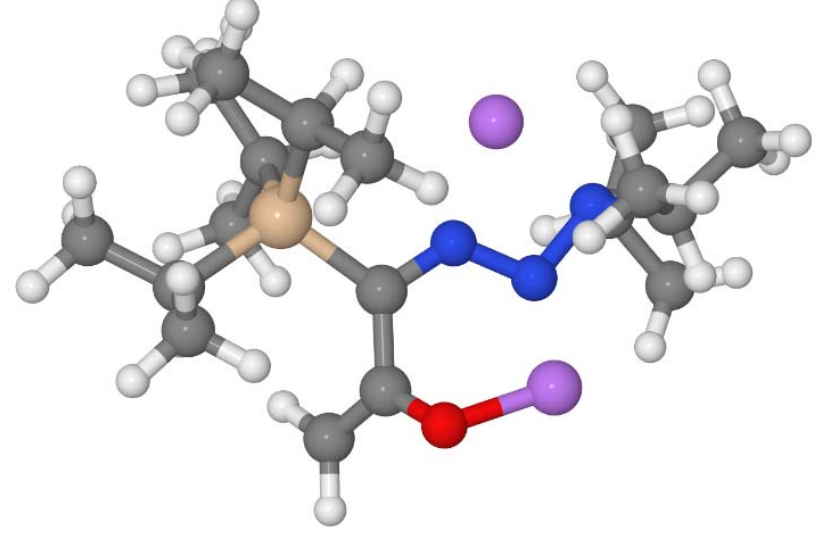
$\begin{array}{llll}\text { C } & -0.43736 & -0.79116 & -0.47933\end{array}$
C $\quad-0.44622 \quad-2.17229 \quad-1.09061$
$\begin{array}{llll}\text { O } & 0.24948 & -3.09305 & -0.47730\end{array}$
$\mathrm{N} \quad 0.67717 \quad-0.20422 \quad-0.12635$
$\begin{array}{llll}\mathrm{N} & 1.88720 & -0.75214 & -0.30127\end{array}$
$\begin{array}{llll}\mathrm{N} & 2.86401 & 0.25498 & 0.08484\end{array}$
$\begin{array}{llll}\text { C } & 3.45208 & 0.81296 & -1.17361\end{array}$
$\begin{array}{llll}\mathrm{H} & 2.57721 & 0.89676 & -1.82329\end{array}$
$\begin{array}{llll}\text { C } & 3.80711 & -0.37191 & 1.04324\end{array}$ 


$\begin{array}{lrrr}\mathrm{H} & 4.21366 & -1.28808 & 0.58780 \\ \mathrm{Li} & 1.93275 & -2.65870 & -0.22778 \\ \mathrm{C} & -1.17054 & -2.38295 & -2.22498 \\ \mathrm{H} & -1.73133 & -1.58299 & -2.69116 \\ \mathrm{H} & -1.19688 & -3.36812 & -2.68015 \\ \mathrm{Si} & -1.93924 & 0.25434 & -0.00508 \\ \mathrm{C} & -3.53720 & -0.72888 & -0.29416 \\ \mathrm{H} & -3.43791 & -1.10486 & -1.31891 \\ \mathrm{C} & -3.61375 & -1.95213 & 0.63118 \\ \mathrm{H} & -3.78040 & -1.65078 & 1.67039 \\ \mathrm{H} & -2.69871 & -2.55120 & 0.59617 \\ \mathrm{H} & -4.44722 & -2.60286 & 0.34293 \\ \mathrm{C} & -4.84057 & 0.07901 & -0.23286 \\ \mathrm{H} & -4.85135 & 0.89749 & -0.95687 \\ \mathrm{H} & -5.01411 & 0.50703 & 0.75758 \\ \mathrm{H} & -5.69531 & -0.56885 & -0.46021 \\ \mathrm{C} & -1.71443 & 0.78299 & 1.82645 \\ \mathrm{H} & -1.02496 & 1.64344 & 1.79044 \\ \mathrm{C} & -1.07939 & -0.29536 & 2.71597 \\ \mathrm{H} & -0.93912 & 0.07847 & 3.73717 \\ \mathrm{H} & -0.11064 & -0.63060 & 2.33929 \\ \mathrm{H} & -1.72331 & -1.17792 & 2.77758 \\ \mathrm{C} & -3.01041 & 1.27896 & 2.48697 \\ \mathrm{H} & -3.53360 & 2.03160 & 1.89314 \\ \mathrm{H} & -2.79992 & 1.71596 & 3.46982 \\ \mathrm{H} & -3.70032 & 0.44511 & 2.64542 \\ \mathrm{C} & -1.86929 & 1.83503 & -1.07094 \\ \mathrm{H} & -0.80577 & 2.11666 & -1.03990 \\ \mathrm{C} & -2.21685 & 1.52655 & -2.53274 \\ \mathrm{H} & -2.08300 & 2.41218 & -3.16427 \\ \mathrm{H} & -3.25973 & 1.20778 & -2.63470 \\ \mathrm{H} & -1.58416 & 0.73158 & -2.93831 \\ \mathrm{C} & -2.67553 & 3.03411 & -0.55725 \\ \mathrm{H} & -2.35795 & 3.34339 & 0.44239 \\ \mathrm{H} & -3.74664 & 2.81666 & -0.51203 \\ \mathrm{H} & -2.54776 & 3.89557 & -1.22327 \\ \mathrm{C} & 4.96969 & 0.54036 & 1.42546 \\ \mathrm{H} & 5.51697 & 0.08224 & 2.25260 \\ \mathrm{H} & 4.60189 & 1.51380 & 1.76425 \\ \mathrm{H} & 5.67719 & 0.69880 & 0.61371 \\ \mathrm{C} & 3.04797 & -0.74995 & 2.31514 \\ \mathrm{H} & 3.71328 & -1.27334 & 3.00557 \\ \mathrm{H} & 2.18939 & -1.39106 & 2.11312 \\ \mathrm{H} & 2.69180 & 0.15394 & 2.82606 \\ \mathrm{C} & 4.00594 & 2.23006 & -1.01680 \\ \mathrm{H} & 4.99165 & 2.26397 & -0.55445 \\ \mathrm{H} & 3.32809 & 2.85493 & -0.42685 \\ \mathrm{H} & 4.09354 & 2.68265 & -2.00819 \\ \mathrm{H} & 4.37323 & -0.12336 & -1.86517 \\ \mathrm{H} & 4.00050 & 0.27357 & -1.30567 \\ \mathrm{Li} & 1.36636 & 1.34198 & -2.85269 \\ & & 0.00304 \\ & & \end{array}$

\section{j. 6-2THF}

$\mathrm{E}=-1417.66714443 \mathrm{au}$

Charge: 0, spin: singlet. 5 lowest frequencies: 20.9800, 29.0583, 42.6346, 47.9230, 53.3092. 


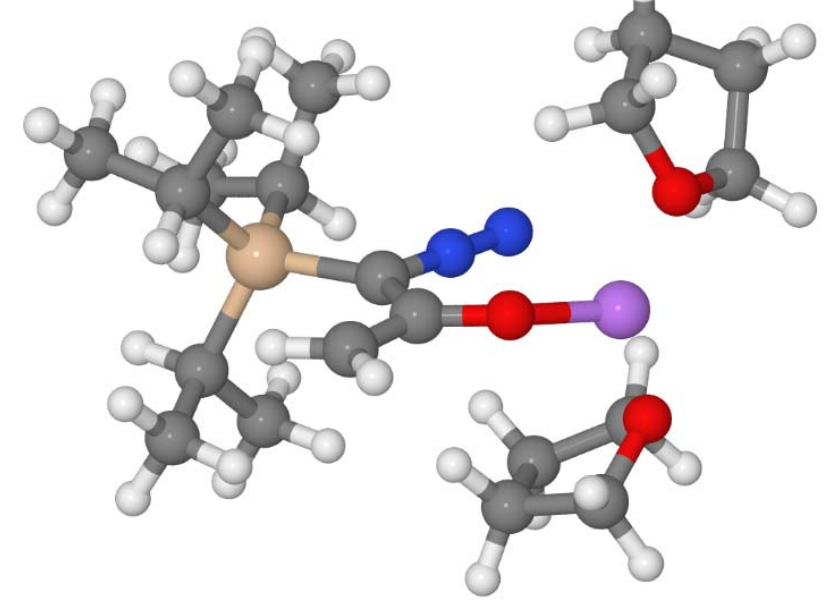

$\begin{array}{llll}\text { C } & -0.89367 & -0.99569 & 2.80177\end{array}$

$\mathrm{H} \quad-1.96109 \quad-0.98538 \quad 2.63321$

H $\quad-0.52090 \quad-1.33618 \quad 3.76058$

$\begin{array}{llll}\text { C } & -0.01192 & -0.58000 & 1.84981\end{array}$

$\begin{array}{llll}\text { O } & 1.27363 & -0.56227 & 1.97005\end{array}$

$\begin{array}{llll}\text { C } & -0.56069 & -0.09513 & 0.52589\end{array}$

$\begin{array}{llll}\mathrm{N} & 0.36695 & 0.22626 & -0.31892\end{array}$

N $\quad 1.18279 \quad 0.51773 \quad-1.05186$

$\begin{array}{llll}\mathrm{Si} & -2.33391 & 0.18146 & -0.08903\end{array}$

C $\quad-3.18914 \quad-1.45903 \quad-0.51257$

$\begin{array}{llll}\mathrm{H} & -4.13873 & -1.16467 & -0.98085\end{array}$

C $\quad-2.10965 \quad 1.14338-1.71702$

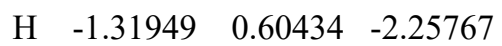

C $\quad-3.35028 \quad 1.12817 \quad-2.62146$

$\begin{array}{llll}\mathrm{H} & -4.20165 & 1.62624 & -2.14990\end{array}$

H $\quad-3.65959 \quad 0.11223 \quad-2.87992$

H $\quad-3.14181 \quad 1.65714 \quad-3.55808$

C $\quad-2.36939-2.24511 \quad-1.54432$

H $\quad-1.41835-2.56762 \quad-1.10758$

H $\quad-2.14411-1.65755 \quad-2.43943$

H $\quad-2.90563 \quad-3.14524 \quad-1.86446$

$\begin{array}{llll}\text { C } & -3.51578 & -2.35382 & 0.68819\end{array}$

$\begin{array}{llll}\mathrm{H} & -4.10037 & -1.83182 & 1.45123\end{array}$

H $\quad-2.60075 \quad-2.72225 \quad 1.16095$

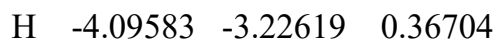

C $\quad-1.62542 \quad 2.58443 \quad-1.50033$

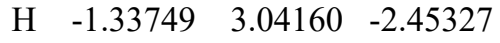

H $\quad-0.76285 \quad 2.64539 \quad-0.82862$

H $\quad-2.42233 \quad 3.19911 \quad-1.07107$

$\begin{array}{llll}\text { C } & -3.31770 & 1.11972 & 1.23599\end{array}$

$\begin{array}{llll}\mathrm{H} & -3.55851 & 0.37484 & 2.00368\end{array}$

$\begin{array}{llll}\text { C } & -4.64830 & 1.64696 & 0.67771\end{array}$

$\begin{array}{llll}\mathrm{H} & -5.22909 & 0.86817 & 0.17350\end{array}$

$\mathrm{H} \quad-4.47978 \quad 2.45680 \quad-0.03843$

$\begin{array}{llll}\mathrm{H} & -5.26835 & 2.04975 & 1.48583\end{array}$

$\begin{array}{llll}\text { C } & -2.53464 & 2.24803 & 1.91849\end{array}$

$\mathrm{H} \quad-2.29142 \quad 3.04841 \quad 1.21448$

$\mathrm{H} \quad-1.60046 \quad 1.88675 \quad 2.35556$

$\mathrm{H} \quad-3.13165 \quad 2.69130 \quad 2.72335$

Li $\quad 2.79574 \quad-0.52430 \quad 1.07680$

$\begin{array}{llll}\text { O } & 3.57957 & 1.11661 & 0.60188\end{array}$

$\begin{array}{llll}\text { C } & 4.37480 & 1.35963 & -0.57470\end{array}$

$\begin{array}{llll}\text { C } & 4.49486 & 2.87333 & -0.66261\end{array}$

$\begin{array}{llll}\text { C } & 3.12498 & 3.31514 & -0.14099\end{array}$

$\begin{array}{llll}\text { C } & 2.88239 & 2.32379 & 0.99003\end{array}$

H $\quad 5.32687 \quad 0.84180 \quad-0.45226$

H $\quad 3.84787 \quad 0.95676 \quad-1.44524$

H $\quad 5.28941 \quad 3.23014 \quad-0.00216$ 


$\begin{array}{lrrr}\mathrm{H} & 4.70118 & 3.21053 & -1.67827 \\ \mathrm{H} & 2.36859 & 3.19243 & -0.91995 \\ \mathrm{H} & 3.10677 & 4.34642 & 0.21108 \\ \mathrm{H} & 1.83034 & 2.07875 & 1.14810 \\ \mathrm{H} & 3.31358 & 2.67179 & 1.93253 \\ \mathrm{O} & 3.16525 & -2.10018 & 0.07378 \\ \mathrm{C} & 2.95566 & -2.23836 & -1.33891 \\ \mathrm{C} & 1.54052 & -2.81914 & -1.49523 \\ \mathrm{C} & 1.20018 & -3.36099 & -0.08613 \\ \mathrm{C} & 2.51916 & -3.23621 & 0.66983 \\ \mathrm{H} & 3.71255 & -2.92438 & -1.73332 \\ \mathrm{H} & 3.08495 & -1.25801 & -1.79587 \\ \mathrm{H} & 0.83175 & -2.04814 & -1.80061 \\ \mathrm{H} & 1.53004 & -3.60550 & -2.25052 \\ \mathrm{H} & 0.83995 & -4.38975 & -0.10442 \\ \mathrm{H} & 0.44507 & -2.73807 & 0.39692 \\ \mathrm{H} & 3.15727 & -4.11537 & 0.52434 \\ \mathrm{H} & 2.40008 & -3.03779 & 1.73456\end{array}$

\section{k. 7-2THF}

$\mathrm{E}=-1710.03921707 \mathrm{au}$

Charge: 0, spin: singlet. 5 lowest frequencies: 19.6956, 26.8860, 27.3027, 37.7180, 43.8550.

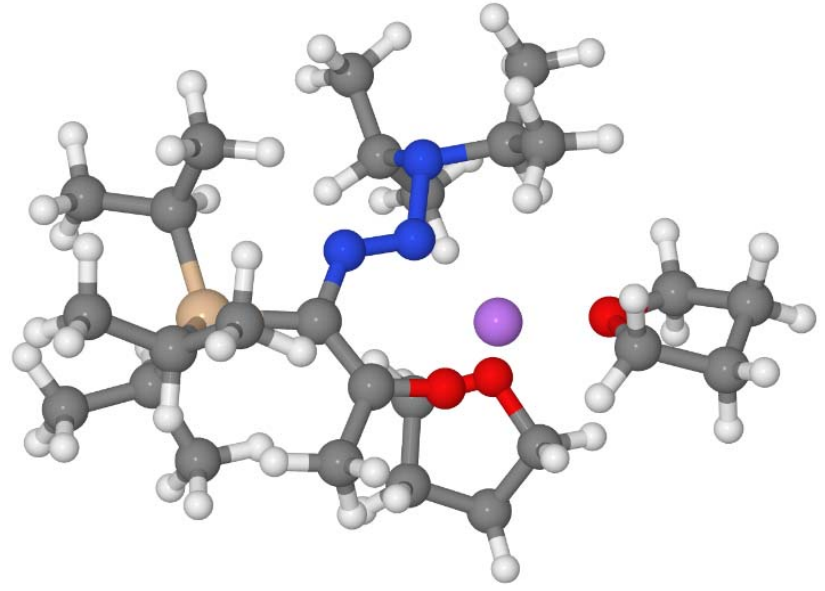
C $\quad-1.22998-0.33682 \quad 0.40836$
$\begin{array}{llll}\text { C } & -0.49484 & -1.49938 & 0.76654\end{array}$
O $\quad 0.76268-1.58354 \quad 0.79952$
$\begin{array}{llll}\mathrm{N} & -0.61961 & 0.86897 & 0.12248\end{array}$
$\begin{array}{llll}\mathrm{N} & 0.62063 & 1.02054 & 0.05035\end{array}$
$\begin{array}{llll}\mathrm{N} & 0.94691 & 2.40251 & -0.23135\end{array}$
C $\quad 0.58452 \quad 2.70703-1.64296$
H $\quad-0.34641 \quad 2.15444-1.78393$
C $\quad 2.35932 \quad 2.57489 \quad 0.15852$
$\begin{array}{llll}\mathrm{H} & 2.98557 & 1.84113 & -0.37687\end{array}$
$\begin{array}{llll}\mathrm{Li} & 1.97592 & -0.42626 & 0.07449\end{array}$
$\begin{array}{llll}\text { C } & -1.22111 & -2.76710 & 1.17049\end{array}$
$\mathrm{H} \quad-2.30517 \quad-2.70060 \quad 1.13985$
$\begin{array}{llll}\mathrm{H} & -0.91120 & -3.03270 & 2.18519\end{array}$
H $\quad-0.90027 \quad-3.57873 \quad 0.51123$
$\begin{array}{llll}\mathrm{Si} & -3.11565 & -0.19865 & 0.27206\end{array}$
$\begin{array}{llll}\text { C } & -4.02246 & -0.87253 & 1.82527\end{array}$

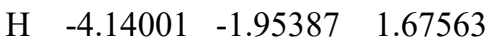
$\begin{array}{llll}\text { C } & -3.21427 & -0.65728 & 3.11295\end{array}$
$\begin{array}{llll}\mathrm{H} & -3.06447 & 0.40978 & 3.30571\end{array}$
H $\quad-2.22576-1.11931 \quad 3.06873$
$\begin{array}{llll}\mathrm{H} & -3.74417 & -1.07560 & 3.97676\end{array}$
$\begin{array}{llll}\text { C } & -5.43681 & -0.30154 & 2.01747\end{array}$
$\begin{array}{llll}\mathrm{H} & -6.07234 & -0.43649 & 1.14018\end{array}$
$\begin{array}{llll}\mathrm{H} & -5.40583 & 0.76741 & 2.24407\end{array}$
$\begin{array}{llll}\mathrm{H} & -5.93077 & -0.79613 & 2.86199\end{array}$ 


\begin{tabular}{|c|c|c|c|}
\hline & 4 & & \\
\hline & -2.73492 & & \\
\hline & & & \\
\hline & & & \\
\hline & & & \\
\hline & & & \\
\hline & & & \\
\hline & & & \\
\hline & & & \\
\hline & & & \\
\hline & & & \\
\hline & & & \\
\hline & -2.9 & & \\
\hline & & & \\
\hline & & & \\
\hline & & & \\
\hline & -5.2 & & \\
\hline & & & \\
\hline & & & \\
\hline & & & \\
\hline & & & \\
\hline & & & \\
\hline & & & \\
\hline & & & \\
\hline & & & \\
\hline & & & \\
\hline & & & \\
\hline & & & \\
\hline & & & \\
\hline & & & \\
\hline & & & \\
\hline & & & \\
\hline & & & \\
\hline & & & \\
\hline & & & \\
\hline & & & \\
\hline & & & \\
\hline & & & \\
\hline & & & \\
\hline & & & \\
\hline & & & \\
\hline & & & \\
\hline & & & \\
\hline & & & \\
\hline & & & \\
\hline & & & \\
\hline & & & \\
\hline & & & \\
\hline & & & \\
\hline & & & \\
\hline & & & \\
\hline & & & \\
\hline & & & \\
\hline & & & \\
\hline & & & \\
\hline & & & \\
\hline & & & \\
\hline & & & \\
\hline & & & \\
\hline & & -4.4 & \\
\hline & & & \\
\hline & & 272052 & 4 \\
\hline
\end{tabular}




\section{7'-2THF}

$\mathrm{E}=-1710.04214157 \mathrm{au}$

Charge: 0, spin: singlet. 5 lowest frequencies: 13.1390, 20.6152, 25.7678, 33.2798, 42.2545.

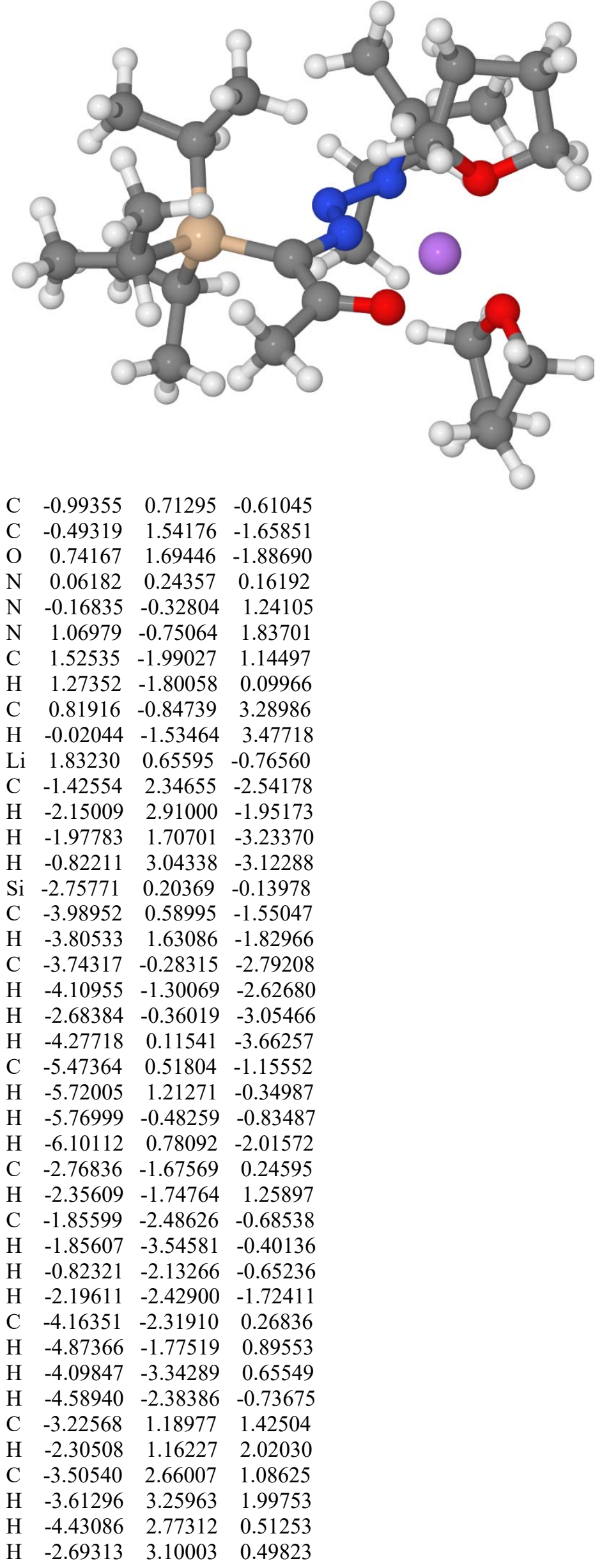




$\begin{array}{rrrr}\mathrm{C} & -4.34298 & 0.59795 & 2.29190 \\ \mathrm{H} & -4.10058 & -0.41500 & 2.62531 \\ \mathrm{H} & -5.29749 & 0.55347 & 1.75889 \\ \mathrm{H} & -4.50001 & 1.20957 & 3.18850 \\ \mathrm{C} & 2.04736 & -1.33956 & 4.05025 \\ \mathrm{H} & 1.86932 & -1.21833 & 5.12157 \\ \mathrm{H} & 2.92477 & -0.74308 & 3.78261 \\ \mathrm{H} & 2.27369 & -2.38996 & 3.87394 \\ \mathrm{C} & 0.44358 & 0.53582 & 3.81938 \\ \mathrm{H} & 0.20282 & 0.47365 & 4.88373 \\ \mathrm{H} & -0.41777 & 0.95080 & 3.29613 \\ \mathrm{H} & 1.28663 & 1.22289 & 3.69591 \\ \mathrm{C} & 3.04410 & -2.15377 & 1.16365 \\ \mathrm{H} & 3.42763 & -2.52182 & 2.11548 \\ \mathrm{H} & 3.52235 & -1.19735 & 0.93887 \\ \mathrm{H} & 3.33326 & -2.87352 & 0.39058 \\ \mathrm{C} & 0.78502 & -3.26119 & 1.56857 \\ \mathrm{H} & 1.07781 & -3.60053 & 2.56489 \\ \mathrm{H} & 1.01036 & -4.06833 & 0.86513 \\ \mathrm{H} & -0.29626 & -3.10059 & 1.56363 \\ \mathrm{O} & 2.59217 & -0.74545 & -1.78552 \\ \mathrm{C} & 3.90608 & -1.24249 & -2.05866 \\ \mathrm{C} & 3.72363 & -2.71290 & -2.49297 \\ \mathrm{C} & 2.19540 & -2.91678 & -2.48637 \\ \mathrm{C} & 1.67767 & -1.48977 & -2.59324 \\ \mathrm{H} & 4.49814 & -1.12237 & -1.15192 \\ \mathrm{H} & 4.35340 & -0.64439 & -2.85893 \\ \mathrm{H} & 4.13074 & -2.86368 & -3.49354 \\ \mathrm{H} & 4.22937 & -3.40128 & -1.81588 \\ \mathrm{H} & 1.86694 & -3.35407 & -1.53973 \\ \mathrm{H} & 1.84823 & -3.55123 & -3.30180 \\ \mathrm{H} & 0.67329 & -1.33043 & -2.19596 \\ \mathrm{H} & 1.71835 & -1.12225 & -3.62589 \\ \mathrm{O} & 3.25182 & 1.61112 & 0.05850 \\ \mathrm{C} & 3.86873 & 2.47017 & -0.92154 \\ \mathrm{C} & 3.47545 & 3.88435 & -0.51953 \\ \mathrm{C} & 3.43594 & 3.76825 & 1.00637 \\ \mathrm{C} & 2.82324 & 2.38614 & 1.19931 \\ \mathrm{H} & 3.50271 & 2.17874 & -1.90821 \\ \mathrm{H} & 4.95286 & 2.32485 & -0.87938 \\ \mathrm{H} & 4.18435 & 4.62958 & -0.88063 \\ \mathrm{H} & 2.48160 & 4.11865 & -0.90881 \\ \mathrm{H} & 2.84576 & 4.54839 & 1.48688 \\ \mathrm{H} & 4.45028 & 3.79395 & 1.41319 \\ \mathrm{H} & 1.72817 & 2.42411 & 1.20043 \\ \mathrm{H} & 3.16027 & 1.87465 & 2.10172\end{array}$

m. 8-4THF

$\mathrm{E}=-2181.86983198 \mathrm{au}$

Charge: 0, spin: singlet. 5 lowest frequencies: 16.5119, 25.9314, 28.8724, 36.0789, 40.6637. 


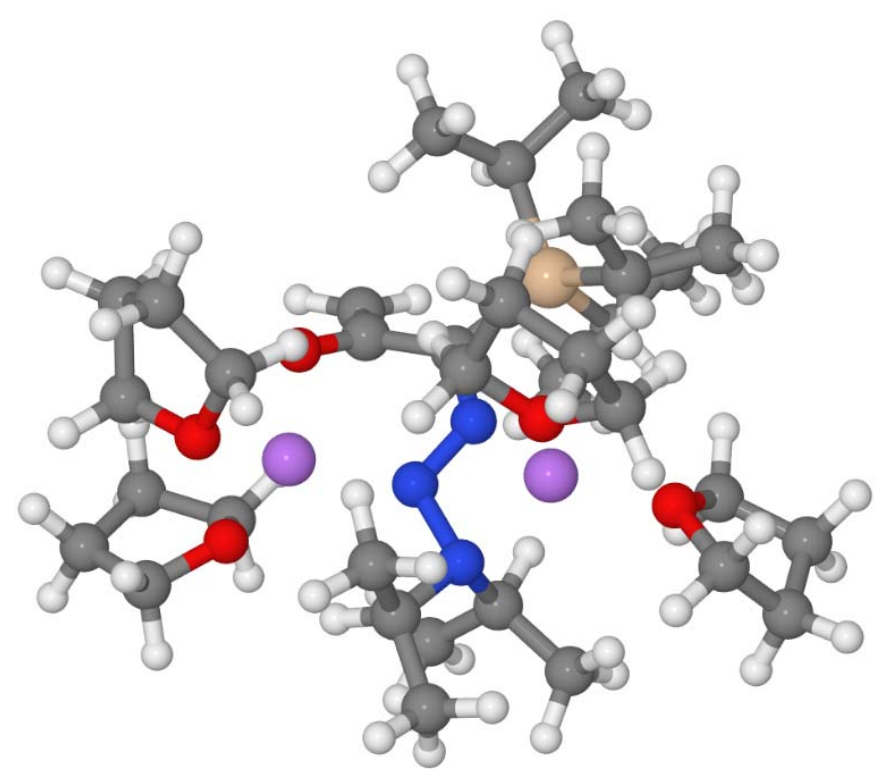

$\begin{array}{llll}\text { C } & 0.07576 & 1.38840 & -0.47202\end{array}$

$\begin{array}{llll}\text { C } & -1.29321 & 1.99669 & -0.69045\end{array}$

$\begin{array}{llll}\mathrm{O} & -2.25997 & 1.59588 & 0.07948\end{array}$

$\begin{array}{llll}\mathrm{N} & 0.22687 & 0.07959 & -0.38681\end{array}$

$\mathrm{N} \quad-0.80548 \quad-0.74251 \quad-0.49158$

$\begin{array}{llll}\mathrm{N} & -0.27757 & -2.10010 & -0.64400\end{array}$

$\begin{array}{lllll}\text { C } & 0.02978 & -2.28219 & -2.09867\end{array}$

$\mathrm{H} \quad 0.59207 \quad-1.37393 \quad-2.33393$

$\begin{array}{llll}\text { C } & -1.31322 & -3.00430 & -0.09061\end{array}$

$\mathrm{H} \quad-2.28482 \quad-2.71643 \quad-0.52053$

$\begin{array}{llll}\mathrm{Li} & -2.62916 & -0.18182 & 0.02938\end{array}$

C $\quad-1.45061 \quad 2.93727 \quad-1.67288$

$\mathrm{H} \quad-0.62325 \quad 3.26800 \quad-2.28511$

$\mathrm{H} \quad-2.41906 \quad 3.40780 \quad-1.81915$

Si $1.67966 \quad 2.37021 \quad-0.58903$

C $\quad 1.38753 \quad 4.21071 \quad-0.15143$

H $\quad 0.89824 \quad 4.64299 \quad-1.03386$

$\begin{array}{llll}\text { C } & 0.43476 & 4.40016 & 1.04093\end{array}$

$\mathrm{H} \quad 0.89702 \quad 4.05616 \quad 1.97065$

$\mathrm{H} \quad-0.50574 \quad 3.86105 \quad 0.91324$

$\begin{array}{llll}\mathrm{H} & 0.19718 & 5.46278 & 1.17200\end{array}$

$\begin{array}{llll}\text { C } & 2.66990 & 5.01745 & 0.10961\end{array}$

H $3.38639 \quad 4.96519 \quad-0.71155$

$\mathrm{H} \quad 3.17755 \quad 4.67155 \quad 1.01460$

$\begin{array}{llll}\mathrm{H} & 2.42490 & 6.07499 & 0.26490\end{array}$

$\begin{array}{llll}\text { C } & 2.95501 & 1.57810 & 0.59739\end{array}$

$\begin{array}{llll}\mathrm{H} & 2.80759 & 0.49834 & 0.45962\end{array}$

$\begin{array}{llll}\text { C } & 2.66873 & 1.88153 & 2.07151\end{array}$

$\begin{array}{llll}\mathrm{H} & 3.30127 & 1.26818 & 2.72387\end{array}$

$\begin{array}{llll}\mathrm{H} & 1.62437 & 1.68901 & 2.33088\end{array}$

$\begin{array}{llll}\mathrm{H} & 2.88275 & 2.92777 & 2.30941\end{array}$

$\begin{array}{llll}\text { C } & 4.42592 & 1.88214 & 0.28128\end{array}$

$\begin{array}{llll}\mathrm{H} & 4.69742 & 1.62882 & -0.74681\end{array}$

$\begin{array}{llll}\mathrm{H} & 5.08721 & 1.31178 & 0.94599\end{array}$

$\mathrm{H} \quad 4.65546 \quad 2.94195 \quad 0.42896$

$\begin{array}{llll}\text { C } & 2.42268 & 2.16842 & -2.35584\end{array}$

$\mathrm{H} \quad 3.00036 \quad 1.23792 \quad-2.26780$

$\begin{array}{llll}\text { C } & 1.39863 & 1.94340 & -3.47619\end{array}$

H $\quad 1.90075 \quad 1.58880 \quad-4.38444$

H $\quad 0.88939 \quad 2.87565 \quad-3.73812$

$\mathrm{H} \quad 0.63142 \quad 1.21432 \quad-3.20175$

C $3.39290 \quad 3.27751 \quad-2.78512$

$\begin{array}{llll}\mathrm{H} & 4.21866 & 3.41661 & -2.08377\end{array}$

H $2.86989 \quad 4.23536 \quad-2.87684$

H $3.82725 \quad 3.05109 \quad-3.76653$ 


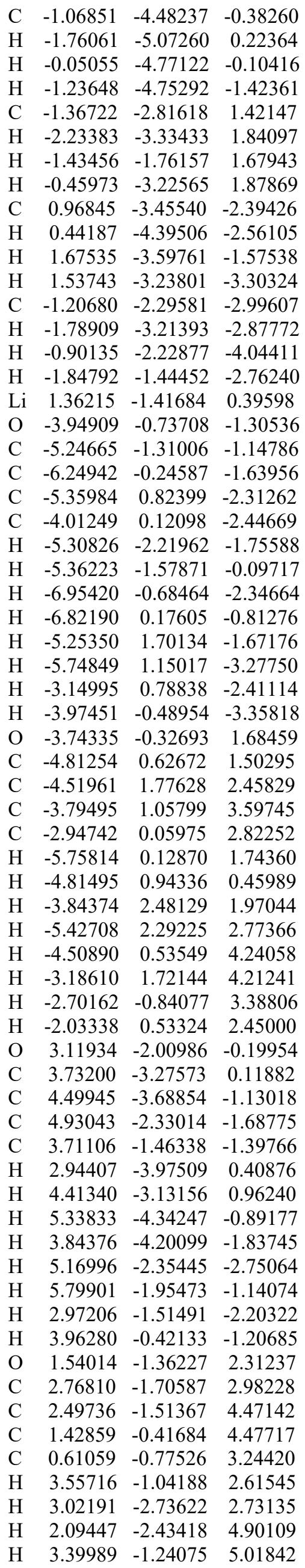

S70 


\section{n. 14}

$\mathrm{E}=-1238.15520831 \mathrm{au}$

Charge: 0, spin: singlet. 5 lowest frequencies: 16.9593, 19.4222, 41.4804, 55.2902, 67.1408.

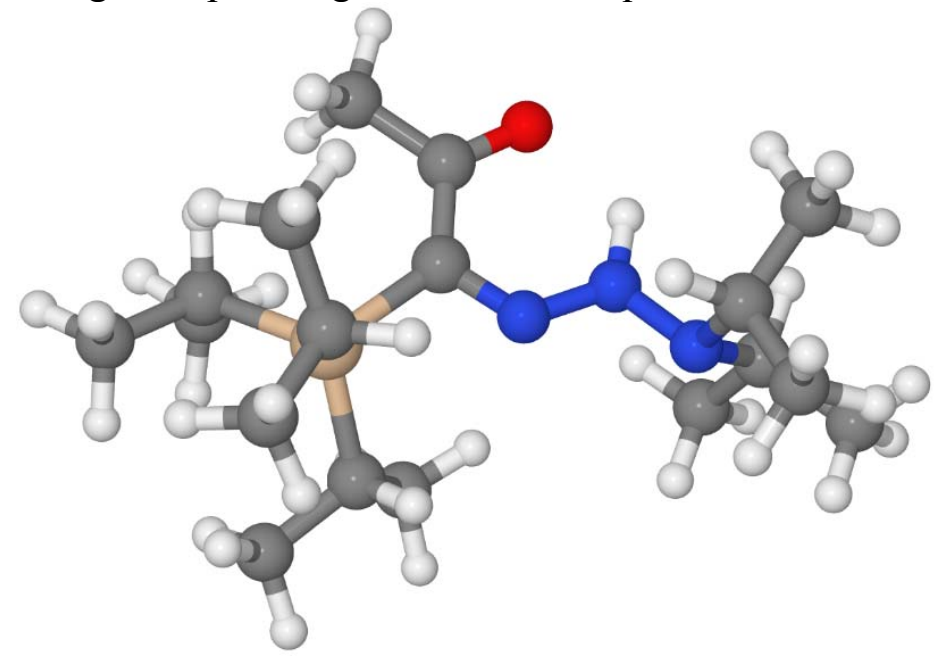
C $\quad-0.39611 \quad 0.80555 \quad-0.46992$
C $\quad-0.27925 \quad 2.12047-1.12146$
$\begin{array}{llll}\mathrm{O} & 0.81000 & 2.56269 & -1.49330\end{array}$
$\begin{array}{llll}\mathrm{N} & 0.68386 & 0.11685 & -0.13792\end{array}$
N $\quad 1.89272 \quad 0.48779 \quad-0.39794$
$\begin{array}{llll}\mathrm{N} & 2.93616 & -0.28829 & 0.11946\end{array}$
$\begin{array}{llll}\text { C } & 3.45699 & 0.39339 & 1.34385\end{array}$
$\begin{array}{llll}\mathrm{H} & 2.54544 & 0.76860 & 1.81646\end{array}$
C $\quad 3.88515 \quad-0.56673 \quad-0.98633$
$\mathrm{H} \quad 4.26168 \quad 0.38396 \quad-1.39660$
$\mathrm{H} \quad 2.07506 \quad 1.37782-0.86756$
C $\quad-1.51020 \quad 2.96949-1.31602$
$\mathrm{H} \quad-2.03993 \quad 3.11093-0.37135$
H $\quad-2.19758 \quad 2.49175 \quad-2.01796$
H $\quad-1.20826 \quad 3.93737 \quad-1.71389$
$\begin{array}{llll}\text { Si } & -1.91725 & -0.15834 & 0.17640\end{array}$
$\begin{array}{llll}\text { C } & -3.54108 & 0.43072 & -0.62024\end{array}$
$\mathrm{H} \quad-3.54555 \quad 1.52063 \quad-0.52485$
C $\quad-3.59593 \quad 0.08378 \quad-2.11632$
$\mathrm{H} \quad-3.74510 \quad-0.99077 \quad-2.25844$
$\mathrm{H} \quad-2.67944 \quad 0.35755 \quad-2.64887$
$\mathrm{H} \quad-4.43250 \quad 0.59623 \quad-2.60433$
C $\quad-4.81382 \quad-0.06388 \quad 0.08475$
$\begin{array}{llll}\mathrm{H} & -4.86500 & 0.27460 & 1.12207\end{array}$

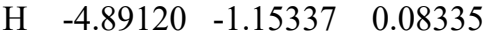
$\begin{array}{llll}\mathrm{H} & -5.69986 & 0.32525 & -0.43007\end{array}$
C $\quad-1.56556-2.00148 \quad-0.16643$

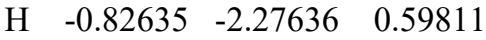
C $\quad-0.93702 \quad-2.28616 \quad-1.53813$

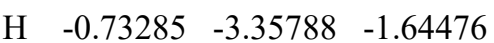

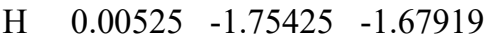
H $\quad-1.61077 \quad-2.00197 \quad-2.35224$
C $\quad-2.79437 \quad-2.90598 \quad 0.02010$

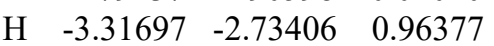
H $\quad-2.49489-3.95968 \quad-0.00551$

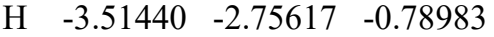
$\begin{array}{llll}\text { C } & -1.90205 & 0.15304 & 2.05371\end{array}$
H $\quad-0.83770 \quad 0.06589 \quad 2.31315$ 


$\begin{array}{rrrr}\mathrm{C} & -2.34028 & 1.58833 & 2.37262 \\ \mathrm{H} & -2.23017 & 1.80330 & 3.44132 \\ \mathrm{H} & -3.39153 & 1.75293 & 2.11359 \\ \mathrm{H} & -1.74255 & 2.32558 & 1.82678 \\ \mathrm{C} & -2.66285 & -0.86230 & 2.91460 \\ \mathrm{H} & -2.26760 & -1.87370 & 2.78975 \\ \mathrm{H} & -3.72994 & -0.88849 & 2.67572 \\ \mathrm{H} & -2.57333 & -0.60386 & 3.97608 \\ \mathrm{C} & 5.07064 & -1.39903 & -0.51103 \\ \mathrm{H} & 5.63616 & -1.73118 & -1.38427 \\ \mathrm{H} & 4.72410 & -2.28442 & 0.02904 \\ \mathrm{H} & 5.75400 & -0.84137 & 0.12740 \\ \mathrm{C} & 3.14709 & -1.31993 & -2.09071 \\ \mathrm{H} & 3.83969 & -1.54642 & -2.90435 \\ \mathrm{H} & 2.31929 & -0.74177 & -2.50383 \\ \mathrm{H} & 2.75104 & -2.26123 & -1.69810 \\ \mathrm{C} & 4.07951 & -0.57890 & 2.34406 \\ \mathrm{H} & 5.09854 & -0.86704 & 2.08839 \\ \mathrm{H} & 3.46966 & -1.48070 & 2.42982 \\ \mathrm{H} & 4.11254 & -0.09565 & 3.32413 \\ \mathrm{C} & 4.36200 & 1.59053 & 1.05258 \\ \mathrm{H} & 5.31919 & 1.28258 & 0.62473 \\ \mathrm{H} & 4.56975 & 2.12544 & 1.98255 \\ \mathrm{H} & 3.88717 & 2.29523 & 0.36336\end{array}$

\section{o. 14'}

$\mathrm{E}=-1238.17051931 \mathrm{au}$

Charge: 0, spin: singlet. 5 lowest frequencies: 26.4250, 35.2156, 42.8975, 63.5605, 66.6967.

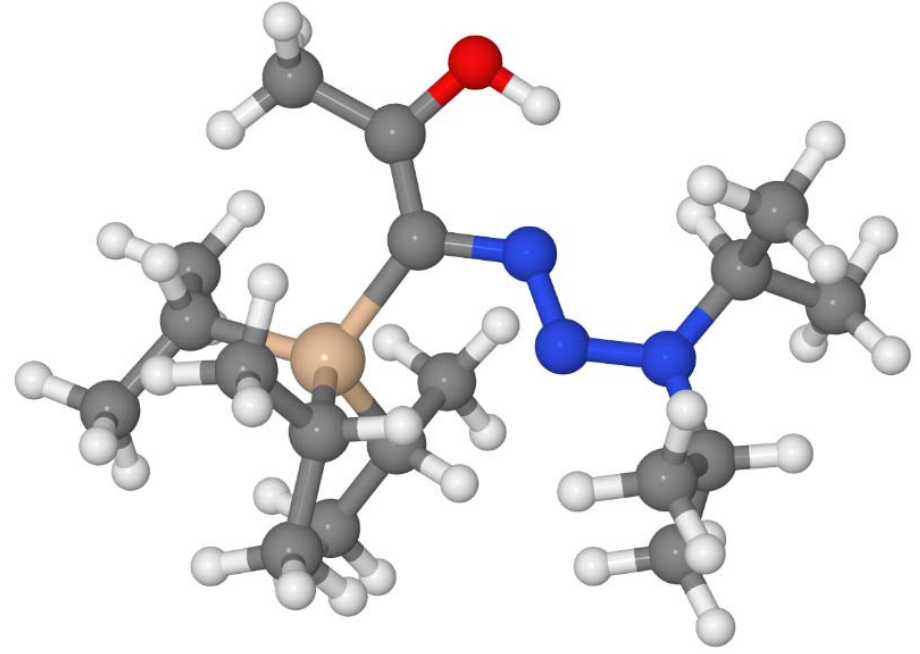
C $\quad-0.43314 \quad 1.32026 \quad 0.15998$
C $\quad-0.68967 \quad 2.65736 \quad 0.34638$
$\begin{array}{llll}\mathrm{O} & 0.34615 & 3.55341 & 0.34536\end{array}$
$\begin{array}{llll}\mathrm{N} & 0.97477 & 1.13209 & -0.00664\end{array}$
N $\quad 1.43012 \quad-0.04872 \quad 0.08412$
$\mathrm{N} \quad 2.74201 \quad-0.20345 \quad-0.13848$
C $\quad 3.63241 \quad 0.93787 \quad-0.44929$
$\begin{array}{llll}\mathrm{H} & 2.97253 & 1.68834 & -0.88942\end{array}$
$\begin{array}{llll}\text { C } & 3.28899 & -1.51719 & 0.27076\end{array}$
H $\quad 4.36839 \quad-1.42360 \quad 0.13149$
$\begin{array}{llll}\text { C } & -1.97522 & 3.37786 & 0.59318\end{array}$
$\mathrm{H} \quad-2.82824 \quad 2.71435 \quad 0.70177$
$\mathrm{H} \quad-2.17925 \quad 4.08299 \quad-0.22440$
$\mathrm{H} \quad-1.88407 \quad 3.97406 \quad 1.51098$
Si $-1.62703 \quad-0.18760 \quad 0.02566$
C $\quad-3.48168 \quad 0.34278 \quad-0.10704$
$\begin{array}{llll}\mathrm{H} & -3.65137 & 1.02518 & 0.73696\end{array}$
$\begin{array}{llll}\text { C } & -3.82234 & 1.10319 & -1.40573\end{array}$
H $\quad-3.78748 \quad 0.43510 \quad-2.27496$ 


$\begin{array}{rrrr}\mathrm{H} & -3.13986 & 1.93666 & -1.60677 \\ \mathrm{H} & -4.84140 & 1.51583 & -1.35697 \\ \mathrm{C} & -4.49172 & -0.80925 & 0.09429 \\ \mathrm{H} & -4.36108 & -1.31673 & 1.05563 \\ \mathrm{H} & -4.42617 & -1.56662 & -0.69413 \\ \mathrm{H} & -5.51793 & -0.41186 & 0.07456 \\ \mathrm{C} & -1.06813 & -1.18833 & -1.53331 \\ \mathrm{H} & -0.16269 & -1.71421 & -1.20257 \\ \mathrm{C} & -0.66145 & -0.30280 & -2.72979 \\ \mathrm{H} & -0.27638 & -0.92512 & -3.55209 \\ \mathrm{H} & 0.12110 & 0.41824 & -2.47031 \\ \mathrm{H} & -1.51401 & 0.26282 & -3.12654 \\ \mathrm{C} & -2.06534 & -2.26340 & -2.01418 \\ \mathrm{H} & -2.38431 & -2.94127 & -1.21484 \\ \mathrm{H} & -1.60456 & -2.88076 & -2.80032 \\ \mathrm{H} & -2.96646 & -1.81424 & -2.44862 \\ \mathrm{C} & -1.34196 & -1.19481 & 1.64820 \\ \mathrm{H} & -0.25315 & -1.13759 & 1.78425 \\ \mathrm{C} & -1.98170 & -0.51036 & 2.87316 \\ \mathrm{H} & -1.66652 & -1.00595 & 3.80382 \\ \mathrm{H} & -3.07817 & -0.55240 & 2.84203 \\ \mathrm{H} & -1.69227 & 0.54559 & 2.95425 \\ \mathrm{C} & -1.70290 & -2.69236 & 1.61022 \\ \mathrm{H} & -1.17434 & -3.22371 & 0.80979 \\ \mathrm{H} & -2.77716 & -2.86114 & 1.46707 \\ \mathrm{H} & -1.42492 & -3.17677 & 2.55892 \\ \mathrm{C} & 3.03261 & -1.82102 & 1.75449 \\ \mathrm{H} & 3.56324 & -2.73688 & 2.04298 \\ \mathrm{H} & 1.96528 & -1.97117 & 1.94887 \\ \mathrm{H} & 3.38781 & -1.00532 & 2.39424 \\ \mathrm{C} & 2.79282 & -2.65009 & -0.63663 \\ \mathrm{H} & 3.30526 & -3.58534 & -0.37934 \\ \mathrm{H} & 2.99330 & -2.42766 & -1.69089 \\ \mathrm{H} & 1.71632 & -2.81052 & -0.51595 \\ \mathrm{C} & 4.26753 & 1.53470 & 0.81654 \\ \mathrm{H} & 4.96195 & 0.82641 & 1.28500 \\ \mathrm{H} & 3.50323 & 1.80715 & 1.55296 \\ \mathrm{H} & 4.83315 & 2.43989 & 0.56136 \\ \mathrm{C} & 4.68083 & 0.56986 & -1.50637 \\ \mathrm{H} & 5.44105 & -0.12390 & -1.12956 \\ \mathrm{H} & 5.20112 & 1.48220 & -1.82216 \\ \mathrm{H} & 4.21162 & 0.12517 & -2.39140 \\ \mathrm{H} & 1.14764 & 3.00687 & 0.17010\end{array}$

